Portland State University

PDXScholar

Winter 3-4-2015

\title{
Modifications to a Cavity Ringdown Spectrometer to Improve Data Acquisition Rates
}

Gregory Alan Bostrom

Portland State University

Follow this and additional works at: https://pdxscholar.library.pdx.edu/open_access_etds

Part of the Optics Commons

Let us know how access to this document benefits you.

\section{Recommended Citation}

Bostrom, Gregory Alan, "Modifications to a Cavity Ringdown Spectrometer to Improve Data Acquisition Rates" (2015). Dissertations and Theses. Paper 2208.

https://doi.org/10.15760/etd.2205

This Dissertation is brought to you for free and open access. It has been accepted for inclusion in Dissertations and Theses by an authorized administrator of PDXScholar. Please contact us if we can make this document more accessible: pdxscholar@pdx.edu. 
Modifications to a Cavity Ringdown Spectrometer to Improve Data Acquisition Rates

by

Gregory Alan Bostrom

A dissertation submitted in partial fulfillment of the requirements for the degree of

Doctor of Philosophy

in

Applied Physics

Dissertation Committee:

Andrew Rice, Chair

Dean Atkinson

Erik Sanchez

Aslam Khalil

Tami Lasseter Clare

Portland State University

2015 


\begin{abstract}
Cavity ringdown spectroscopy (CRDS) makes use of light retention in an optical cavity to enhance the sensitivity to absorption or extinction of light from a sample inside the cavity. When light entering the cavity is stopped, the output is an exponential decay with a decay constant that can be used to determine the quantity of the analyte if the extinction or absorption coefficient is known. The precision of the CRDS is dependent on the rate at which the system it acquires and processes ringdowns, assuming randomly distributed errors. We have demonstrated a CRDS system with a ringdown acquisition rate of $1.5 \mathrm{kHz}$, extendable to a maximum of $3.5 \mathrm{kHz}$, using new techniques that significantly changed the way in which the ringdowns are both initiated and processed. On the initiation side, we combined a custom high-resolution laser controller with a linear optical feedback configuration and a novel optical technique for initiating a ringdown. Our optical injection "unlock" method switches the laser off-resonance, while allowing the laser to immediately return to resonance, after terminating the unlock, to allow for another ringdown (on the same cavity resonance mode). This part of the system had a demonstrated ringdown initiation rate of $3.5 \mathrm{kHz}$. To take advantage of this rate, we developed an optimized cost-effective FGPA-based data acquisition and processing system for CRDS, capable of determining decay constants at a maximum rate of $4.4 \mathrm{kHz}$, by modifying a commercial ADC-FPGA evaluation board and programming it to apply a discrete Fourier transform-based algorithm for determining decay constants. The entire system shows promise with a demonstrated ability to determine gas concentrations for $\mathrm{H}_{2} \mathrm{O}$ with a measured concentration accuracy of $\pm 3.3 \%$. The system achieved an
\end{abstract}


absorption coefficient precision of $0.1 \%$ (95\% confidence interval). It also exhibited a linear response for varying $\mathrm{H}_{2} \mathrm{O}$ concentrations, a $2.2 \%$ variation $(1 \sigma)$ for repeated measurements at the same $\mathrm{H}_{2} \mathrm{O}$ concentration, and a corresponding precision of $0.6 \%$ (standard error of the mean). The absorption coefficient limit of detection was determined to be $1.6 \times 10^{-8} \mathrm{~cm}^{-1}$ (root mean square of the baseline residual). Proposed modifications to our prototype system offer the promise of more substantial gains in both precision and limit of detection. The system components developed here for faster ringdown acquisition and processing have broader applications for CRDS in atmospheric science and other fields that need fast response systems operating at high-precision. 


\section{Dedication}

To my wife, Alison, without whose continual support and encouragement I could never have come close to accomplishing this.

To my daughter, Naomi, who has only ever known me to be a graduate student-may she always appreciate physics the way she does now.

To my Dad, whom I think would have really liked to have seen this, and to my Mom, who has been proud enough of me for both of them. 


\section{Acknowledgements}

I would like to of course thank my advisor, Dr. Andrew Rice, for guiding me for almost 8 years on this project. And to Dr. Dean Atkinson, who has helped me for just as long, was nice enough to let me take apart his equipment, and has provided more useful and practical advice than I can remember.

I am indebted to my friend, Jeremy Parra for providing countless hours of brainstorming, motivation, and much needed distraction.

I would like to thank Aarisa Smith, who helped me with processing the system's data, and who was brave enough to run the system herself.

I would also like to thank the Xilinx University Program for providing access to the Xilinx ISE® Design Suite through a donation to Portland State University. 


\section{Table of Contents}

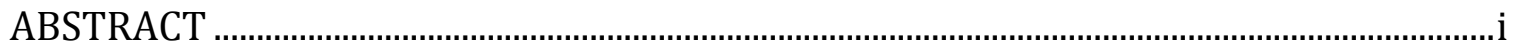

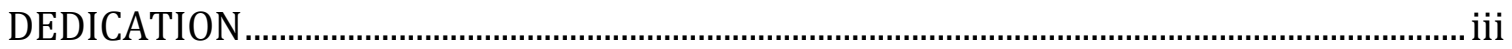

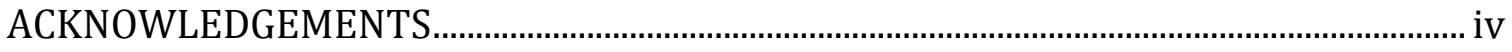

LIST OF TABLES ….................................................................................................................. vii

LIST OF FIGURES .................................................................................................................. vii

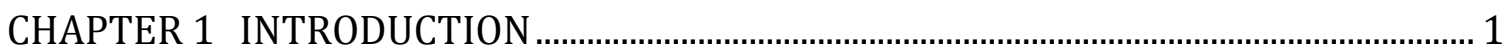

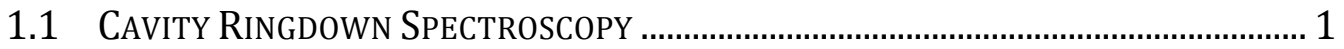

1.2 APPLICATIONS TO ATMOSPHERIC SCIENCE ............................................................ 4

1.3 REVIEW OF OUR PREVIOUS WORK......................................................................... 5

1.4 Current State of The Science For High-Precision/High-SPEed CRDS....10

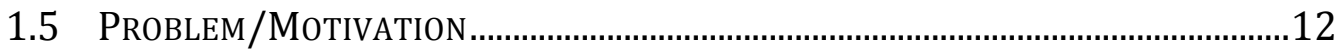

CHAPTER 2 SYSTEM DESIGN AND APPROACH.................................................................

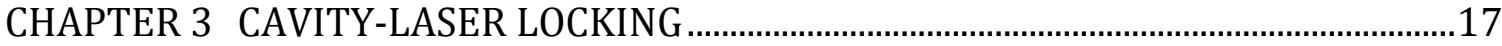

3.1 COHERENT OPTICAL FEEDBACK THEORY ..........................................................17

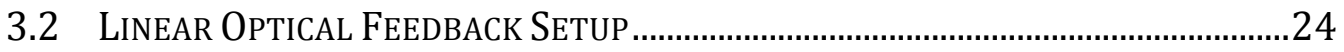

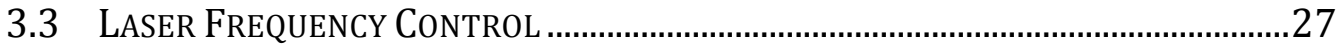

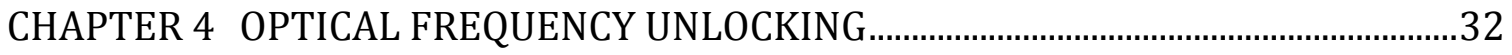

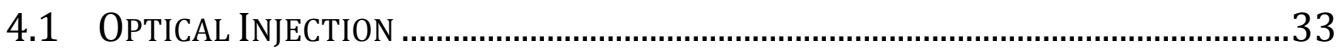

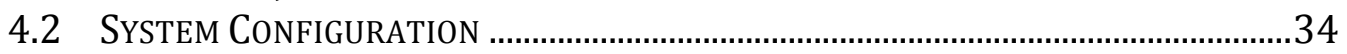

4.3 Frequency Shift Performance (Frequency Domain) ..................................38

4.4 Ringdown PERformance (Time-Domain) ......................................................40

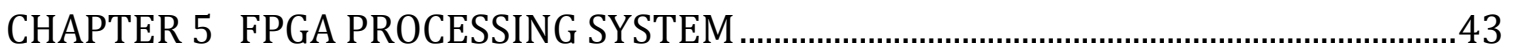

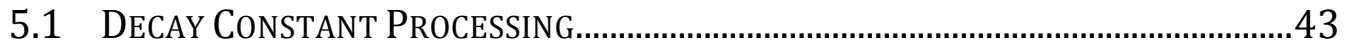

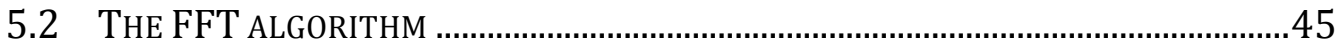

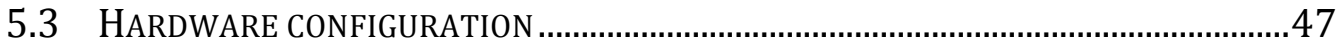

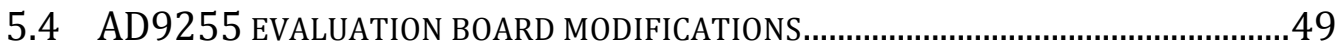

5.5 FPGA EVALUATION BOARD CONFIGURATION .........................................................50

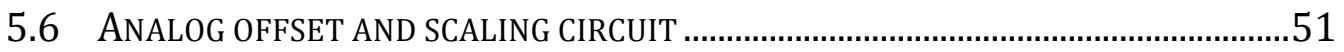

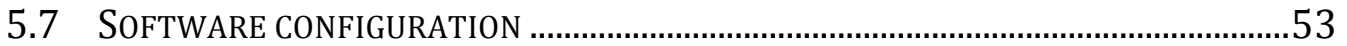

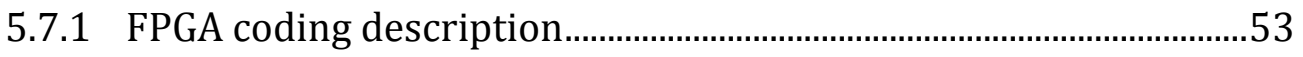

5.7.2 Arduino Due storage and post-processing .........................................55

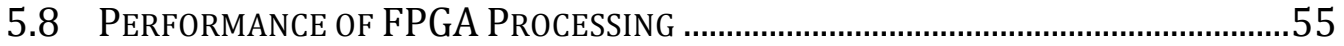

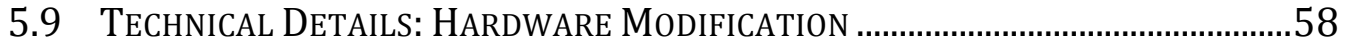

5.9.1 ADC evaluation board modifications ...................................................58

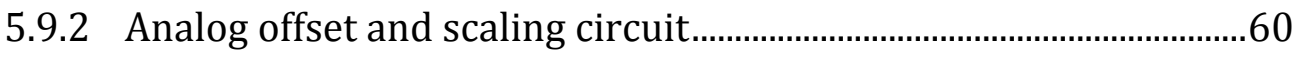


5.9.3 FPGA board modifications and pin mapping ....................................60

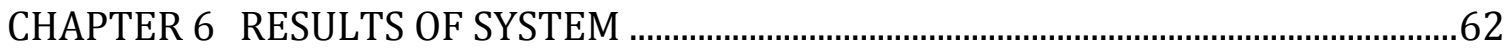

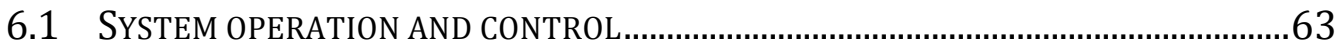

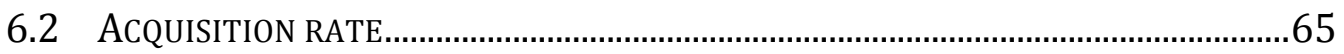

6.3 COMPARISON WITH THEORETICAL ABSORPTION SPECTRA.....................................66

6.4 PRECISION AND LIMIT OF DETECTION ……............................................................70

6.5 CONCENTRATION MEASUREMENT REPEATABILITY AND LINEARITY .........................76

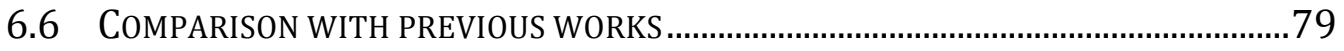

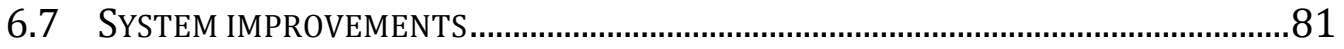

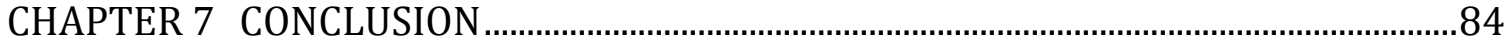

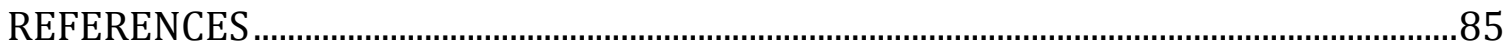

APPENDIX A: FFT ALGORITHM FOR DECAY CONSTANT CALCULATION ......................89

APPENDIX B: OPTICAL HETERODYNE DETECTION …........................................................94

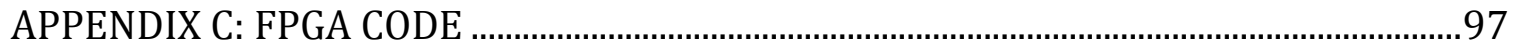

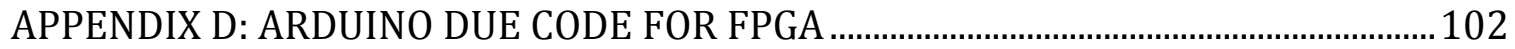

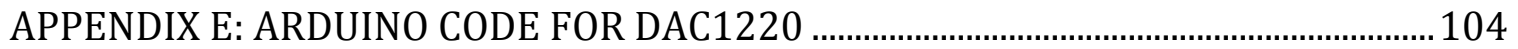

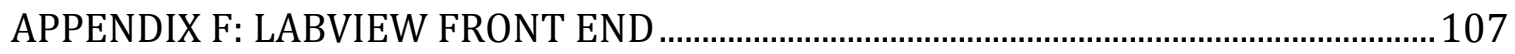




\section{List of Tables}

TABLE 1 Arduino Due to FPGA Translation. The names in Columns 2-5 correspond to those given in the Evaluation board User's Guide. The Arduino Due GPIO pins correspond to the labeling on its circuit board... 


\section{List of Figures}

Figure 1 Cavity ringdown traces for a baseline (blue) and with an absorbing sample (green). The faster decay is caused by the absorption, which increases the time constant from that of an empty cavity. The change in the time constant is then related to the concentration of the absorbing molecules in the cavity.

Figure 2 CRDS system used in previous work. A tunable diode laser's beam was sent into a high-finesse cavity. Once sufficient intensity was built up inside the cavity (a proportional signal is detected by Detector..

Figure 3 A typical scan showing Lorentzian line fit with background subtraction for the ${ }^{13} \mathrm{CH}_{4}$ peak (Peak A). Green circles: Measured CRD spectra; Black, dashed: Background for ${ }^{13} \mathrm{CH}_{4}$ peak; Red dash: Lorentzian fit to ${ }^{13} \mathrm{CH}_{4}$ peak (Peak A) with background subtracted; Blue: combination of Lorentzian fit and background. Peak $\mathrm{C}$ is an $\mathrm{H}_{2} \mathrm{O}$ line which made a significant contribution to the background of the ${ }^{12} \mathrm{CH}_{4}$ peak (Peak B).................... 8

Figure $4 \delta^{13} \mathrm{C}$ enrichment results for 14 October 09 , along with a linear bestfit. The best-fit line has a slope of 2.0 , and an $\mathrm{R}^{2}=0.996$.

Figure 5 Overall system block diagram. Major components are discussed in the following sections. The main laser is injected into the high-finesse cavity. A pulsed laser induces a frequency shift on the laser once triggered. The cavity output is measured with a photodetector, with the output voltage processed using an ADC-FPGA system. A desktop computer with LabView code controls the entire system. The OHD frequency analyzer system and the digital oscilloscope are ancillary diagnostic tools, not necessary for spectroscopy

Figure 6 Effect of optical feedback on laser output. The dashed line shows the laser output frequency if there were no optical feedback (i.e., it is a 1:1 line). The solid line shows the output frequency in the presence of feedback, and the shallow slope around 0 (which represents a mode of the cavity) indicates the frequency locking-the laser output does not move far from resonance. In this case, the locking range would be \pm 8.0 $\mathrm{MHz}$ (the extremes of $\omega_{\text {free }}$ ) and the slope is $120 \mathrm{rad} / \mathrm{rad}$.

Figure 7 Effect of laser-cavity interaction. Linewidth of the main laser is shown with the cavity aligned (blue) and then when the cavity output mirror is mis-aligned (red). The main laser linewidth is significantly narrowed when the cavity output is included in the feedback. A 10-point moving average is shown on the misaligned cavity to better represent the peak. 
Figure 8 Extended Laser-Cavity interaction with induced ringdowns. The long-term increase (and then decrease) in peak voltage demonstrates that the laser frequency remained close to the cavity mode for extended periods of time (10 $\mathrm{ms}$ in this diagram). The drop outs were ringdowns induced by the pulsed laser, as described in Section 2.4. Without locking the build-up would only exist for on the order of $100 \mu \mathrm{s}$.

Figure 9 Plot of data taken for wavelength vs. Temperature for the Main Laser. Three datasets were taken on three different days. The average slope for these three days is $-0.55 \mathrm{~cm}^{-1} /{ }^{\circ} \mathrm{C}$.

Figure 10 Circuit Diagram for DAC 1220 output for filtering and attenuating the signal. The buffered output of the DAC1220 evaluation board was passed through a RC low-pass filter and a voltage divider.

Figure 11 Main Laser Wavenumber as a function of current-control applied voltage. The quadratic fit parameters are shown, along with the $95 \%$ confidence interval for those values. An improved fit for this is given in Section 6.3, based on comparison with a published spectrum.

Figure 12 Diagram of the system. The main laser is injected into the optical cavity for the ring-down measurement. A pulsed laser is aligned to inject light back into the main laser. A simple pulsing/triggering circuit is used to activate the pulsed laser. A tunable diode laser and a secondary detector are used to detect frequency shifts in the main laser using OHD for initial characterization (dash-boxed region is not a permanent part of the system). A digital oscilloscope measures the cavity output and optical heterodyne signals. The faraday rotator and polarizer control optical feedback to the main laser to enable locking to the cavity. The quarterwave plate reduces coherent reflections from the pulsed laser.

Figure 13 Circuit diagram for pulsed laser. The circuit is an astable multivibrator circuit using a 555 timer IC. Pulse width and duty cycle (in monostable mode) are controlled by adjusting $\mathrm{Ra}$ and $\mathrm{R}_{\mathrm{b}}$. The values in the diagram correspond to $90 \mu \mathrm{s}$.

Figure 14 Frequency shift from OIU. Three datasets are shown. The FFT of the OHD signal of the laser before (dashed), during (solid black), and after (solid gray). The peak signal before and after overlap, when the pulsed laser is on, the frequency is shifted $20 \mathrm{MHz}$ 
Figure 15 Induced frequency shift as a function of the wavelength difference between the Main Laser and the Pulse Laser. In the main figure, the Main Laser wavelength is adjusted by changing its temperature. The average of 3 different measurements taken at each wavelength is shown, with error bars indicating the max and min. Inset: The frequency shift as a function of Main Laser current, with laser temperature fixed. The range covers approximately $34 \mathrm{GHz}(0.2 \mathrm{~nm})$. Again, average is shown for 3 different measurements at each current level, error bars indicate max and min.

Figure 16 A single ring-down event induced by the pulsed laser frequency shifting the main laser captured on an 8-bit digital oscilloscope (dots). To reduce quantization error, the data was resampled at a rate of 1:5. The exponential fit using a Levenberg-Marquardt algorithm is shown (solid line), as well as the residual to the fit (inset)

Figure 17 Using the frequency shift for ringdowns. Two consecutive ringdowns are shown, indicating that the main laser locks back to the cavity after the pulse laser is turned off. The dashed line is the digital pulse sent to the laser.

Figure 18 Block Diagram of DAQ system, whose main components are the AD9255 ADC evaluation board and the co-requisite HSC-EVALC board containing the FPGA. The blocks in gray are the additional circuits or evaluation board modifications discussed in the text.

Figure 19 Comparison of FPGA captured ringdown with and without the ADC front end circuit. (a) The gray line shows the photodetector output (triggered at $440 \mathrm{mV}$ ) passed directly to ADC evaluation board, which exhibits distortion due to the low common mode voltage set by the AD9255 amplifier. (b) This shows a signal at the same trigger level passed through the scaling and offset circuit, which eliminates the phase reversal distortion. The front end expands the range of valid data in both voltage and in the number of points available for determining the decay constant. Exponential curve fits (black lines) are shown in both (a) and (b) to highlight the distortion, and the residual to the fit is shown in the inset.

Figure 20 Near infrared spectrum of $\mathrm{H}_{2} \mathrm{O}$ obtained using decay constants calculated on the FPGA. The average of the samples taken at each wavenumber are shown as dots, with error bars indicating \pm one standard deviation for that point. Voigt lineshapes were applied to each of the two peaks, and the solid line indicates the fit, while the residuals are shown above the spectrum.

Figure 21 Circuit Diagram of the amplifier input path to the ADC. The rectangles around the labels indicate components that were already on the circuit board, and were not modified. The other resistors and the 18 $\mathrm{pF}$ capacitors need to be installed on the board. 
Figure 22 Diagram of the analog offset and scaling diagram to the AD9255 Evaluation Board. An adjustable offset is subtracted from the output of the photodetector, and the result is scaled using a voltage divider so that the ringdown is within the voltage limits of the ADC. The resistors in the offset and scaling dashed rectangles are made using the center tap of a 5 $\mathrm{k} \Omega$ and $200 \Omega$ variable resistor, respectively.

Figure 23 Plot of the v CRDS output (black dots), the Lorentzian fit (gray line), and the expected spectrum using the HITRAN database (blue line). To get the CRDS output wavenumber, the output voltage was scaled by following: $v(\mathrm{~V})=-0.009 \mathrm{~V}^{2}-0.127 \mathrm{~V}+7640.887$, where these coefficients are well within the error limits of the $v(\mathrm{~V})$ curve fit in Section 2.3. The residuals from the Lorentzian fit (gray line) are shown in the inset.

Figure $24 \mathrm{H}_{2} \mathrm{O}$ spectra including the Lorentzian fit and the residual. We can estimate precision $\_$, by multiplying the standard error by 1.96 .

Figure 25 Repeated measurements of absorption coefficient at a constant laser current. The program collected data repeatedly in $100 \mathrm{~ms}$ blocks. The graph shows the average for each $100 \mathrm{~ms}$ period, with the error bars indicating the $95 \%$ confidence interval. The dashed line shows the overall average absorption coefficient for the 64 periods.

Figure 26 Histogram of the 5682 absorption measurements taken at a constant laser current. Also shown is a fit for a Gaussian distribution. Statistics indicate that in this case, the relative standard deviation is $4.5 \%$, and the precision is $1.6 \times 10^{-8} \mathrm{~cm}^{-1}$.

Figure 27 Allan Variance of repeatability data, which suggests that the sensitivity of the overall system is best when the number of averages is 200-300 data points, or approximately $300 \mathrm{~ms}$. This could be a result of the maximum number of averages stored being 150, such that over time the variation increases.

Figure 28 Estimating the limit of detection of the system. Using the standard deviation of the residuals at the baseline (shown in the inset). In this case, we chose the region to the left of the smaller peak (enclosed in the blue box) to represent the baseline.

Figure 29 Repeatability of the Lorentzian fit peak height (which is proportional to the concentration). The data shows 50 trials at a nearly constant $\mathrm{H}_{2} \mathrm{O}$ concentration. The statistics show an average absorption of $2.9 \times 10^{-6} \mathrm{~cm}^{-1}$ (shown as the dashed line) and standard deviation of $6.4 \mathrm{x}$ $10^{-8} \mathrm{~cm}^{-1}$, yielding a relative standard deviation of $2.2 \%$. The error bars indicate the RMSE of the Lorentzian fit for each data concentration measurement. 
Figure 30 Correlation of CRDS system with LI-840 NDIR measurements. During this trial the LI-840 sampled the same air as the CRDS cavity. A linear fit (red line) between the two shows the CRDS responds to changes in $\mathrm{H}_{2} \mathrm{O}$ concentration linearly. The data was taken over approximately a 5 hour period.

Figure 31 Q-Q plot of residuals for the line fit. The residuals were normalized and plotted against a Gaussian probability distribution (circles). The solid line is a 1:1 line, and deviations of the residuals from this line indicate non-normal distribution. Significantly on the low end there are several outliers that deviate significantly from a Gaussian distribution. 


\section{Chapter 1 Introduction}

\subsection{Cavity Ringdown Spectroscopy}

Cavity ringdown spectroscopy (CRDS) is one of several cavity-enhanced absorption techniques that make use of the multiple reflections of light inside a highfinesse optical cavity to increase the interaction of the light with gases inside the cavity. In our continuous-wave implementation of the method, when the frequency of the laser matches a mode of the cavity, the light intensity inside the cavity (and therefore at the output photodiode at the end of the cavity) increases. At a pre-selected threshold level, a feedback circuit shuts off the light going to the cavity, and this initiates the decay (ringdown) of the signal from within the cavity. The output signal $S(t)$ - the small fraction transmitted by the output mirror-is proportional to the intensity within the cavity, and is therefore given by an exponential. CRDS uses the exponential decay of light intensity emitted from a high finesse resonant cavity to measure the concentration of gases within the cavity, which absorb at that specific frequency. To relate the output to the absorption inside, we can define the output signal:

$$
S(t)=S_{0} e^{-\beta t}
$$

where $S_{0}$ is the level of the detected signal at time $t=0$, and $\beta$ is the decay constant $(1 / \tau$, where $\tau$ is the ring-down time). $\beta$ can be decomposed into $\beta=\beta_{\text {sample }}+\beta_{0}$, where $\beta_{\text {sample }}$ $\left(1 / \tau_{\text {sample }}\right)$ is the contribution from the absorption strength of the sample, and $\beta_{0}\left(1 / \tau_{0}\right)$ is the background absorption of the light due to mirror losses, background gases, etc. In 
theory, $\beta_{0}$ is constant and can be obtained by measuring a ring-down without the sample of interest in the cavity. Subtracting out $\beta_{0}$ leaves $\beta_{\text {sample }}$ from which we determine the sample concentration using the Beer-Lambert Law (see Section 6.3). If we consider only the transverse electromagnetic wave mode $\left(\right.$ known as $\left.^{\mathrm{T}} \mathrm{EM}_{00}\right)$ of the cavity, the frequency spacing is given by the usual standing wave requirement that the cavity length be an integer multiple of half the wavelength, so that the frequency spacing between cavity modes is $\Delta v=c /(2 L)$. Therefore it is possible to scan the laser frequency, determine decay constants at successive longitudinal modes, and obtain an absorption spectrum. Concentrations can then be obtained by matching measured absorption peaks to a database such as HITRAN $^{1}$ (see Section 6.3), or to a higher degree of accuracy in calibration using reference gases of known concentration.

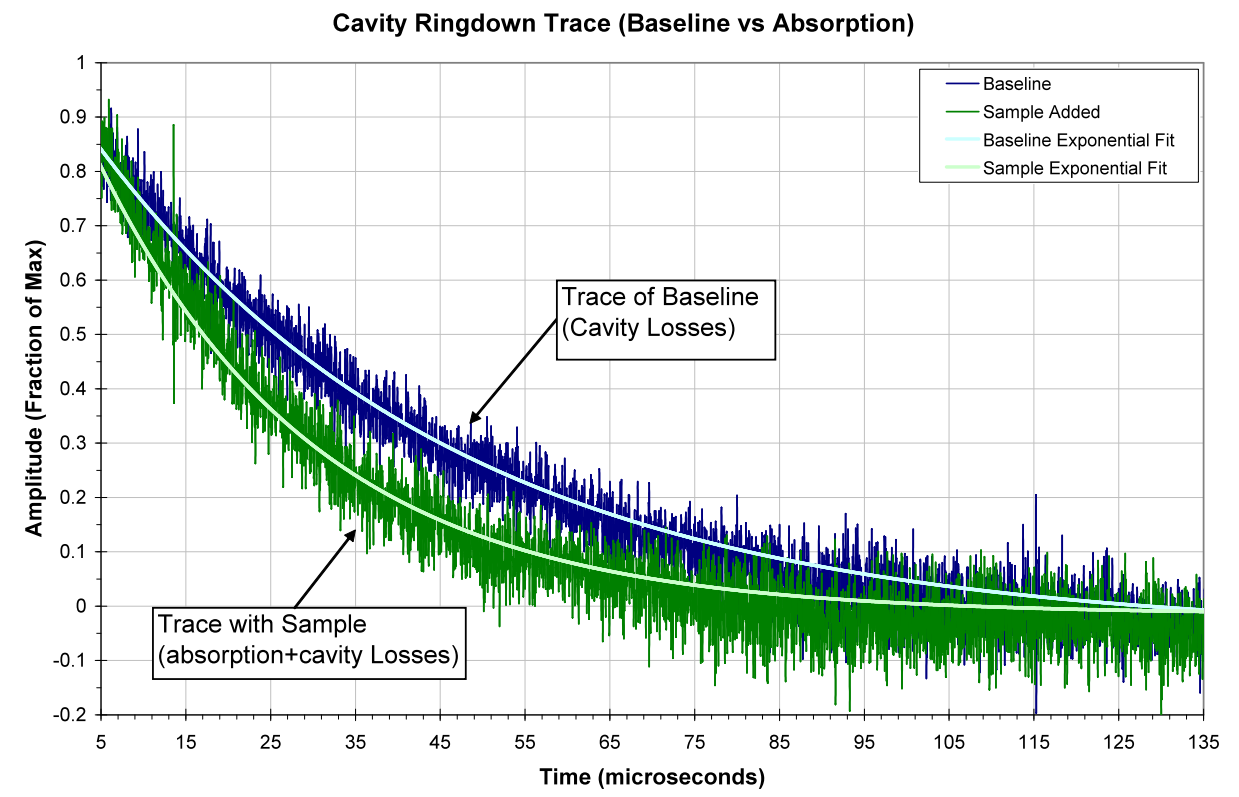

Figure 1 Cavity ringdown traces for a baseline (blue) and with an absorbing sample (green). The faster decay is caused by the absorption, which increases the time constant from that of an empty cavity. The change in the time constant is then related to the concentration of the absorbing molecules in the cavity. 
There are several historical reviews of CRDS, including a particularly applicable one by Paldus and Kachanov ${ }^{2}$. The CRDS began as a way to measure the properties of the high-reflectivity dielectric mirror coatings developed in the 1980's, with typical loss values of parts-per-million (ppm), and was originally known as cavity ringdown reflectometry. Those first credited with using it for measuring gas-phase absorption spectra, and for designating it as CRDS, were O'Keefe and Deacon, who measured forbidden (very weak) oxygen transition bands ${ }^{3}$. Their original system used a pulsed laser CRDS (P-CRDS), but the quest for better sensitivities led to increased interest in the development of continuous wave (CW) CRDS (CW-CRDS), which use CW lasers with much narrower linewidths and finer wavelength/frequency resolution.

CRDS, and other cavity-enhanced techniques such as cavity enhanced absorption spectroscopy (CEAS) ${ }^{4}$, and integrated cavity output spectroscopy (ICOS) $)^{5}$ offered orders of magnitude increase in sensitivity of traditional absorption spectroscopy, including multi-pass-cell absorption spectroscopy ${ }^{6-8}$. As a result, it found its place in a number of applications needing high frequency resolution and absorption (and extinction) sensitivity. In addition to high precision gas measurements ${ }^{9,10}$, it has been used for the measurement of ethane in human breath $^{11}$, chemical dynamics such as radical formation $^{12,13}$, photolysis ${ }^{14,15}$, and flame analysis ${ }^{16}$.

Commercial CRDS systems became available in the 2000's, with Tiger Optics, LLC., (based on technology developed at Princeton by Kevin Lehmann) and Picarro, Inc. (based on technology developed at Stanford by Barbara Paldus and co-workers). Tiger Optics has a wide range of products for measuring gas-phase concentrations of carbon dioxide $\left(\mathrm{CO}_{2}\right)$, water $\left(\mathrm{H}_{2} \mathrm{O}\right)$, methane $\left(\mathrm{CH}_{4}\right)$, and many others down to the parts-per- 
billion (ppb) and in some cases parts-per-trillion (ppt) level. The Picarro instruments are capable of measuring a similar, but slightly smaller, range of gases at similar detection limits. Picarro, Inc., also now offers instruments that measure the isotopic composition (see Section 1.3) of $\mathrm{H}_{2} \mathrm{O}, \mathrm{CH}_{4}, \mathrm{CO}_{2}$, and nitrous oxide $\left(\mathrm{N}_{2} \mathrm{O}\right)$. Picarro achieves their precision using a 3-mirror "ring" cavity, precision control of pressure and temperature and a patented wavemeter enabling the system to lock the laser to the desired frequency. (Thus, they only need to measure the peak absorptions of the isotopologues, rather than an entire spectrum). Tiger Optics' systems are 2-mirror linear cavities, and use automatic laser tuning either with or without a reference cell (depending on the model) to ensure the laser is centered on the peak absorption. They appear to use a similar technique of only measuring the absorption peak and a baseline to determine gas concentration.

\subsection{Applications to atmospheric science}

As a sensitive absorption spectroscopy technique, CRDS has applications in atmospheric science typical of other spectrometers. Many of these applications deal with the measurement of concentrations of greenhouse gases, pollutants, and aerosols. Typical measurements include concentration studies of $\mathrm{CH}_{4}{ }^{17}, \mathrm{CO}_{2}{ }^{18}$ and $\mathrm{H}_{2} \mathrm{O}^{19}$, as well as nitrogen dioxide $\left(\mathrm{NO}_{2}\right)^{20}$ and nitrate $\left(\mathrm{NO}_{3}\right)^{21}$. Several studies have used CRDS to measure not absorption but extinction to measure aerosols ${ }^{22-24}$. More recent applications are measuring greenhouse gas fluxes using $\mathrm{CRDS}^{25}$.

Another of the more recent applications of CRDS is the study of the isotopic composition of greenhouse gases ${ }^{26-29}$, which can provide a better understanding of the processes that produce (sources), consume (sinks), and/or transport greenhouse gases ${ }^{30-32}$. 
While the precision of CRDS is not yet equal to that of the more common IRMS system $^{33,34}$, the portability and speed of CRDS spectrometers make them better suited to in-situ measurements of isotope ratios ${ }^{29,35,36}$. Furthermore, CRDS systems can be significantly less expensive than IRMS systems, but are less flexible in their ability to measure isotope ratios of different gases.

\subsection{Review of our previous work}

Our previous work focused on this last application, specifically measuring the carbon isotope ratio of $\mathrm{CH}_{4}$ using a cavity ringdown spectrometer. Measuring the isotopic composition of $\mathrm{CH}_{4}$ in the environment is of value because each source and each sink imparts a characteristic isotopic "fingerprint" to emitted or consumed $\mathrm{CH}_{4}$. This isotopic analysis of atmospheric $\mathrm{CH}_{4}$ requires determining the ratios of the concentrations of the

$\mathrm{CH}_{4}$ isotopologues (e.g., ${ }^{12} \mathrm{CH}_{4},{ }^{13} \mathrm{CH}_{4}$, and $\mathrm{CH}_{3} \mathrm{D}$ ) to a high degree of accuracy and precision. This analysis provides insight into $\mathrm{CH}_{4}$ sources and sinks, as well as their relative impacts on atmospheric concentrations.

Measurements of the isotopic composition are expressed in terms of isotope ratios using the $\delta$-notation, in which isotopic ratios (rare isotope/most abundant isotope) are expressed relative to an internationally recognized standard reference ratio. For ${ }^{13} \mathrm{C} /{ }^{12} \mathrm{C}$ and $\mathrm{D} / \mathrm{H}$ we have the following:

$$
\delta^{13} C=\left(\left(\left[\frac{\left.{ }^{13} C\right]}{\left[{ }^{12} C\right]}\right)_{\text {sample }} /\left(\frac{\left[{ }^{13} C\right]}{\left[{ }^{12} C\right]}\right)_{\text {standard }}-1\right) \cdot 1000\right.
$$




$$
\delta D=\left(\left(\frac{[D]}{[H]}\right)_{\text {sample }} /\left(\frac{[D]}{[H]}\right)_{\text {standard }}-1\right) \cdot 1000
$$

Where $\left(\left[{ }^{13} C\right] /\left[{ }^{12} C\right]\right)_{\text {sample }}$ and $\left(\left[{ }^{13} C\right] /\left[{ }^{12} C\right]\right)_{\text {standard }}$ are the ratio of the concentrations of ${ }^{13} \mathrm{C}$ to ${ }^{12} \mathrm{C}$ for the sample and standard, respectively; and $([D] /[H])_{\text {sample }}$ and $([D] /[H])_{\text {standard }}$ are the ratio of concentrations of $\mathrm{D}$ to $\mathrm{H}$ for the sample and standard, respectively. The factor of 1000 indicates that values are given in "per mil" (\%o) the adopted notation for reporting isotope ratios ( $\delta$ values). The standard for $\delta^{13} \mathrm{C}$ is Vienna Peedee Belemnite (VPDB) which has a defined ${ }^{13} \mathrm{C} /{ }^{12} \mathrm{C}$ ratio of 0.0112372 ; the standard for $\delta \mathrm{D}$ is Vienna Surface Mean Ocean Water (VSMOW) which has a defined D/H ratio of $0.00015574^{37,38}$.

Our previous work focused on this last application, specifically measuring the isotope ratio of $\mathrm{CH}_{4}$ demonstrated the measurement of $\delta^{13} \mathrm{C}-\mathrm{CH}_{4}$ using a $\mathrm{CRD}$ spectrometer with a near-IR tunable diode laser. Figure 2 shows a diagram of our instrument. For a full description of our analytical system, see Bostrom ${ }^{30}$. Briefly, the beam from a tunable diode laser (New Focus, Model 8324, 1280-1340 nm) passed through an acousto-optic modulator, to enable shutting off the laser input when recording the ringdown event, to an optical cavity. The flow cell is enclosed by a blown pyrex cylinder and sealed by adjustable mirror mounts, producing an $87 \mathrm{~cm}$ optical cavity, with $\mathrm{TEM}_{00}$ longitudinal modes spaced roughly $172 \mathrm{MHz}$ apart. One of the cavity mirrors was mounted on a piezo-electric transducer stage, which was modulated at approximately 15 $\mathrm{Hz}$ to aid in the mode matching of the laser and cavity. Relative frequency measurements (used to linearize the frequency axis) were made using the transmission of a low-finesse 
etalon (whose output is approximately sinusoidal) by matching the data to a theoretical curve. $\mathrm{CH}_{4}$ was enriched dynamically through mass flow controllers on prepared samples of ${ }^{12} \mathrm{CH}_{4}$ and ${ }^{13} \mathrm{CH}_{4}$ with concentrations of approximately $2000 \mathrm{ppm}$ and $40 \mathrm{ppm}$, respectively. Ringdowns were sampled on a PC using a high-speed (50 MSPS) data acquisition card (Gage, CS8012), and Labview code to determine the absorbance. Postprocessing to determine concentration based on the absorbance spectra was performed using Matlab code.

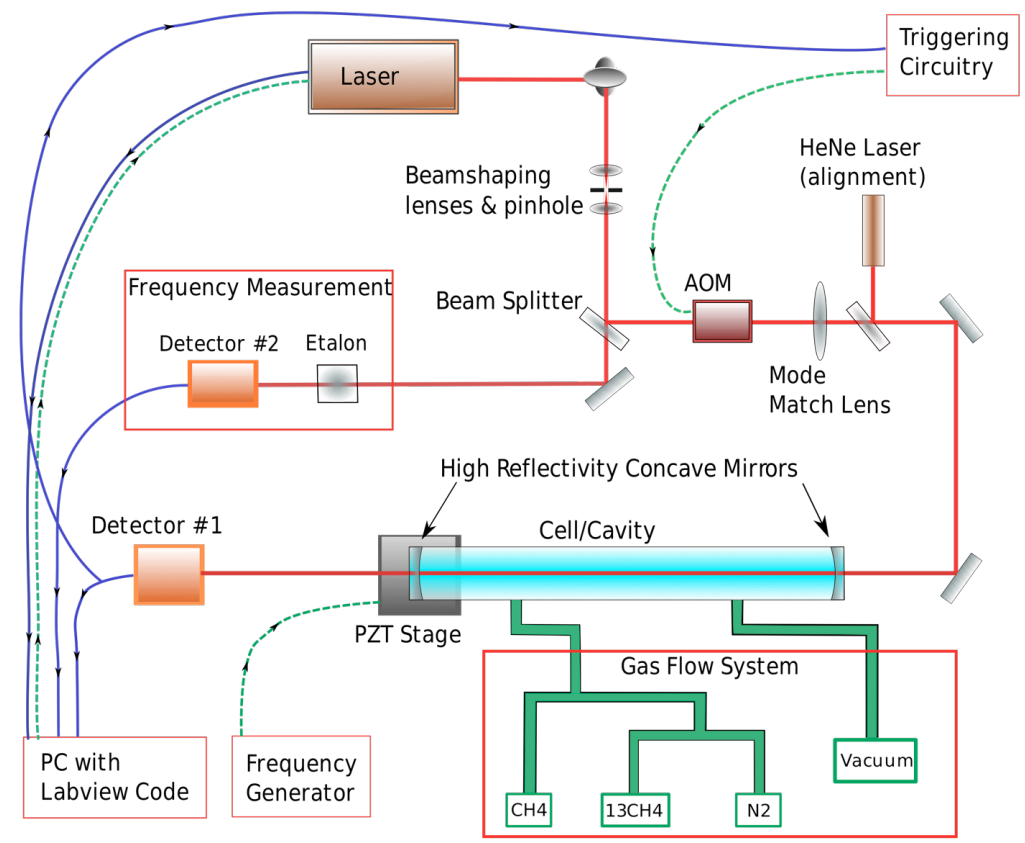

Figure 2 CRDS system used in previous work. A tunable diode laser's beam was sent into a high-finesse cavity. Once sufficient intensity was built up inside the cavity (a proportional signal is detected by Detector $\# 1$ ), an AOM interrupted the beam and started the ringdown event. A low-finesse etalon was used to measure relative frequency, since the tuning of the laser was non-linear.

Isotopic ratio measurements only require a stable relative measurement of the concentrations of the isotopologues, but CRDS allows us to obtain $\delta^{13} \mathrm{C}, \delta \mathrm{D}$, and $\mathrm{CH}_{4}$ concentration with the same instrument. Our initial studies focused on $\delta^{13} \mathrm{C}$ over $\delta \mathrm{D}$ due 
to larger absorption signals resulting from a higher abundance of ${ }^{13} \mathrm{C}$ relative to $\mathrm{D}$. A complete absorption spectrum is shown in Figure 3. We used a Lorentzian line-shape fit to both the background peaks (which were subtracted from the desired peak) and the ${ }^{13} \mathrm{CH}_{4}$ peak (See Section 6.3). This process was repeated for a background and several dynamically enriched $\mathrm{CH}_{4}$ samples. A plot of the expected (based on the concentration of our prepared samples and their flow rates), and the measured $\delta^{13} \mathrm{C}$ (using the total absorption of the ${ }^{13} \mathrm{CH}_{4}$ peak) is shown in Figure 4.

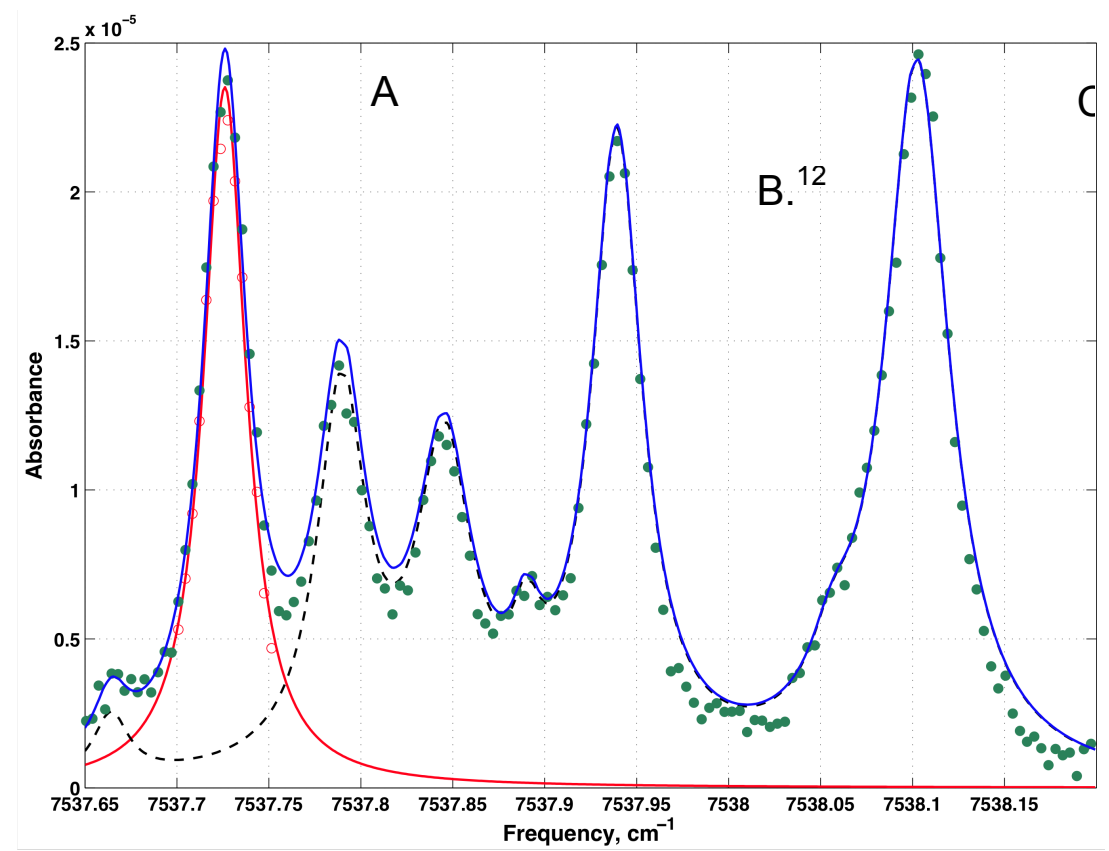

Figure 3 A typical scan showing Lorentzian line fit with background subtraction for the ${ }^{13} \mathrm{CH}_{4}$ peak (Peak A). Green circles: Measured CRD spectra; Black, dashed: Background for ${ }^{13} \mathrm{CH}_{4}$ peak; Red dash: Lorentzian fit to ${ }^{13} \mathrm{CH}_{4}$ peak (Peak A) with background subtracted; Blue: combination of Lorentzian fit and background. Peak $\mathrm{C}$ is an $\mathrm{H}_{2} \mathrm{O}$ line which made a significant contribution to the background of the ${ }^{12} \mathrm{CH}_{4}$ peak (Peak B). 


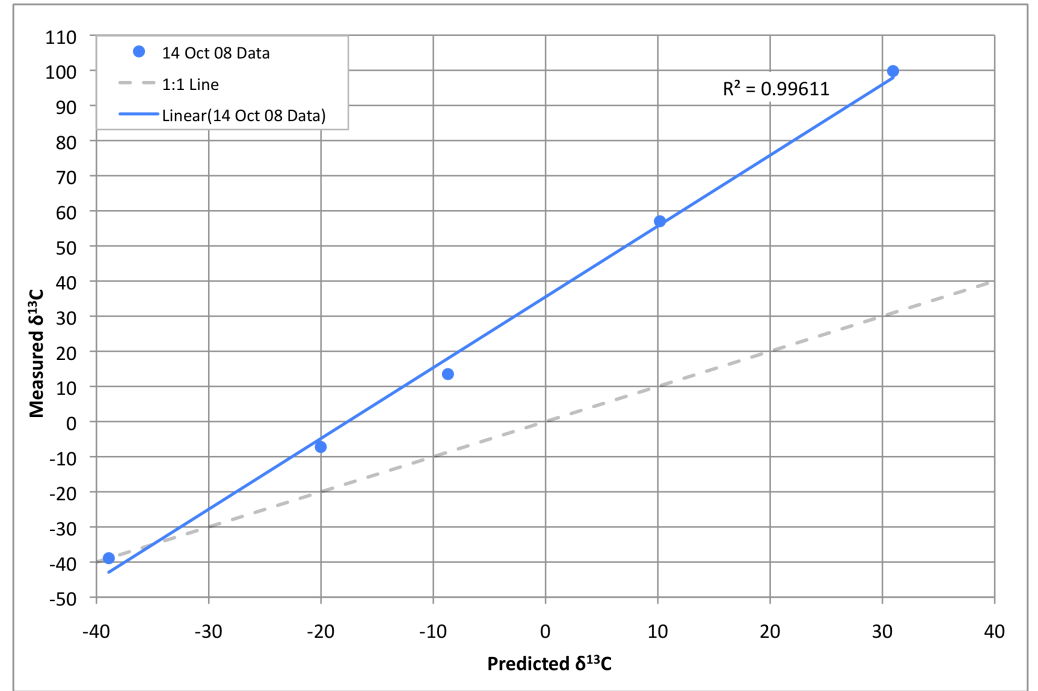

Figure $4 \delta^{13} \mathrm{C}$ enrichment results for 14 October 09 , along with a linear best-fit. The best-fit line has a slope of 2.0 , and an $\mathrm{R}^{2}=0.996$.

We were able to successfully measure the isotope ratio of artificially enriched $\mathrm{CH}_{4}$, with an estimated precision of $4-5 \%$. While our results showed high linearity $\left(\mathrm{R}^{2}=\right.$ 0.996), the ${ }^{12} \mathrm{CH}_{4}$ peak exhibited significant variations (it was expected to remain constant), and therefore we were unable to use it as a measure for ${ }^{12} \mathrm{CH}_{4}$ concentration to a high degree of precision. It also greatly affected our ${ }^{13} \mathrm{C} /{ }^{12} \mathrm{C}$ determination. The reason was most likely a result of temperature variations in the cavity over the course of the experiment and the influence of a varying $\mathrm{H}_{2} \mathrm{O}$ peak (Peak $\mathrm{C}$ ) due to a small leak that was later discovered (and subsequently repaired) in the gas inlet system.

Given the results from the prototype system, we determined that our focus needed to shift to significantly improving the precision and acquisition speed of the CRDS system in general, before we could make further gains in isotope ratio measurements. The system was 4 orders of magnitude from our target precision of $0.5 \%$ at ambient $\mathrm{CH}_{4}$ 
concentrations $(\sim 2 \mathrm{ppm})$. As a result, it was apparent that incremental changes to the system would not be sufficient, but rather a major redesign of the setup was required. We therefore revisited the current research on CRDS sytem design for guidance.

\subsection{Current State of the Science for High-Precision/High-Speed CRDS}

There has been continued effort by the CRDS community to improve the performance of CRDS systems, since increasing levels of precision could lead to the application of CRDS to a larger set of problems (e.g., high precision concentration measurements, isotope ratio measurements, and flux measurements). Often, the sensitivity of absorption spectroscopy is specified in terms of the minimum detectable absorption coefficient. The absorption coefficient is the fractional absorption per unit length, typically given in units of $\mathrm{cm}^{-1}$. For comparison between systems, most authors measure the residuals to the nonlinear fit of absorption coefficient, and use the average root-mean-square (rms) of the residuals at the baseline as their sensitivity. The sensitivity of CRDS systems generally depends on the number of ringdowns averaged to make the measurement, the mechanical stability of the optical cavity, and the stability of the coupling of the laser to the cavity.

Here we highlight work that made improvements to CRDS systems in terms of acquisition speed or precision, or both. In 1997, Romanini et.al. ${ }^{39}$, achieved a $200-\mathrm{Hz}$ ringdown acquisition rate by using a linear fit to the logarithm of the data signal, and achieved a sensitivity of $1 \times 10^{-9} \mathrm{~cm}^{-1}$. In 1998, Paldus et.al. ${ }^{40}$, used optical feedback (see Section 3.1) for enhanced laser-cavity coupling and collected data at $256 \mathrm{~Hz}$. They argued that this was limited by the data acquisition hardware, but claimed their maximum 
ringdown initiation rate was $50 \mathrm{kHz}$. Notably they averaged the ringdowns prior to determining the time constant, which is not the same, or as accurate, as averaging the time constants. They quoted a sensitivity of $5 \times 10^{-9} \mathrm{~cm}^{-1}$. Chen et. al. ${ }^{29}$ reported a sensitivity of $1.9 \times 10^{-12} \mathrm{~cm}^{-1}$ using 100 cycles of 50 averages with an effective ringdown acquisition rate of $12 \mathrm{~Hz}(30 \mathrm{~Hz}+50 \mathrm{~ms} /$ ringdown in processing time). Their system used a PZT to match the cavity mode to the laser frequency, using a low frequency oscillation for searching and high-frequency dither for acquisition. Motto-Ros et.al. ${ }^{41}$ achieved a $0.6 \mathrm{~cm}^{-1}$ spectrum covering 90 cavity modes in only $140 \mathrm{~ms}$, and obtained a 50-point average of each mode in 7 seconds (this is an effective ringdown acquisition rate of $640 \mathrm{~Hz}$ ). Their system also used optical feedback to improve laser-cavity coupling. Since their design swept through each mode successively, their system's sensitivity was limited by the number of samples at each mode they could capture before mechanical instabilities offset any improvement by added decay constant averaging. Their system achieved a sensitivity of $5 \times 10^{-10} \mathrm{~cm}^{-1}$. Crosson et. al. ${ }^{35}$ used a commercial instrument from Picarro, Inc., with a specified ringdown acquisition rate of $100 \mathrm{~Hz}$, an estimated sensitivity of $1.6 \times 10^{-10} \mathrm{~cm}^{-1}$ and a $1-\mathrm{Hz}$ concentration acquisition rate. One of the highest ringdown acquisition rates was achieved by Orr and $\mathrm{He}^{42}$. The first version of their system ${ }^{43}$ used optical heterodyne detection (OHD) to achieve a maximum ringdown acquisition rate of $500 \mathrm{~Hz}$ and achieved a sensitivity of $3.0 \times 10^{-9} \mathrm{~cm}^{-1}$, with 0.6 seconds per spectral data acquisition point. They subsequently followed up with a faster system ${ }^{42}$ and achieved a $5-\mathrm{kHz}$ ringdown acquisition rate by averaging the logarithmic demodulation of the OHD ringdown using analog circuitry. Their sensitivity for this system was $8 \times 10^{-10} \mathrm{~cm}^{-1}$. Long, et.al. ${ }^{44}$ used OHD combined with electronic feedback 
for laser-cavity locking to obtain a sensitivity of $4 \times 10^{-10} \mathrm{~cm}^{-1}$. Butler et.al. ${ }^{23}$ achieved a ringdown acquisition rate of $1.5 \mathrm{kHz}$ using an optical feedback system to measure aerosols rather than perform concentration measurements, so no comparison in sensitivity was stated, or could be determined.

\subsection{Problem/Motivation}

The previous system described in Section 1.3 lacked the precision sensitivity necessary to perform isotope ratio measurements on $\mathrm{CH}_{4}$ at ambient concentrations. Significant improvements needed to be made in order to achieve that objective, which shifted the focus of the current work to develop novel techniques to improve the performance of CRDS systems in general. First, since the standard error of the mean should decrease as $\sqrt{N}$, a significant factor affecting the precision of the system is the speed at which ringdown data can be collected in a CRDS system and converted into absorption $^{45,39}$ Second, reducing the overall time required to obtain a concentration measurement can improve overall system performance by reducing the impact of longterm drift in temperature, pressure, and other environmental factors. It could also enable the system to be applied to measurements that exhibit more rapidly changing concentrations, such as flux measurements. Finally, these improvements should reduce the overall cost of the system to enable CRDS to become more ubiquitous, by further expanding their potential applications. Therefore, the major focus of this work was to find cost-effective methods to improve the speed at which ringdowns can be collected and evaluated in CRDS. This required an investment in and re-examination of the fundamental optics and electronics of CRDS systems. To that end, we have developed 
novel techniques that make significant gains in acquisition speed while simultaneously providing significant cost-savings over conventional methods. 


\section{Chapter 2 System design and approach}

In our previous work, the precision of the instrument was constrained by several factors, the most significant of which was the speed of acquisition. The system collected ringdowns at a rate of approximately $10 \mathrm{~Hz}$. This limitation was a result of 2 major factors: (1) the time it took for the laser output frequency to overlap with the cavity resonance mode, and (2) the speed at which the digitized signal was collected and processed on the computer. The time to acquire a spectrum with reasonable precision was 30-40 minutes. Secondary consequences of this were lower numbers of ringdowns available to average at each frequency, thermal variations in the cavity which significantly altered the relative absorption of the different peaks, and an inability of the system to detect short timescale concentration changes. The intent of my research was then to seek significant improvements in the CRDS system's decay time acquisition speed, as well as reducing the cost and complexity by employing more readily available components into the system.

The first change involved replacing the tunable diode laser $(>\$ 20 \mathrm{k})$ with a lowcost optical-communication-grade distributed feedback laser $(\sim 25$ diode laser $+\sim \$ 500$ for mount, temperature controller, and current controller). The laser stability and linewidth of these lasers are significantly worse than the external-cavity tunable diode laser, but by making use of optical feedback from the cavity mirrors, it was possible to not only improve the stability and linewidth of the new laser, but also to keep the laser locked to the cavity for longer periods of time, allowing significant increases in the rate at 
which we acquired ringdowns. Precision computer control of the laser frequency was accomplished using an inexpensive (\$50) digital-to-analog converter (DAC) evaluation board.

The second improvement eliminated the acousto-optic modulator used to switch off the injection of light into the cavity (which initiates the ringdown). The acousto-optic modulator was another costly device $(\sim \$ 3-5 \mathrm{k})$, required precise alignment to work effectively, and interfered with optical feedback techniques used to keep the laser onresonance with the cavity. The AOM was replaced with a novel system that used optical injection of a secondary pulsed laser $(\sim 25$ for the diode laser $+\$ 300$ for mount and temperature controller) into the probe laser to shift it off-resonance and initiate the ringdown ${ }^{46}$. This technique avoids the thermal effects resulting from modulating the primary laser's current directly ${ }^{41}$, and allows the laser to come back into resonance at the same cavity mode for rapid acquisition of multiple ringdowns.

The third improvement was to replace the PC-based data acquisition system ( $\$ 6 \mathrm{k})$ with a high-speed analog-to-digital converter (ADC) coupled with a field programmable gate array (FPGA) and a microcontroller (total cost $<\$ 900)$ to process the ringdown in near-real time using a discrete Fourier transform (DFT) algorithm, which increased the acquisition and processing rate of ringdowns by 2 orders of magnitude at a significantly reduced cost. The details of each of these modifications, as well as the overall system design, are discussed in the Chapters 3-5, while Chapter 6 discusses the overall performance of the new system.

The design of this second-generation prototype spectrometer is shown in Figure 5. The layout is similar to our original design shown in Figure 2 of Section 1.3. Major 
changes (followed by the sections in which they are discussed) are: the main laser and control circuitry (Chapter 3), the acousto-optic modulator was removed and replaced with a pulsed laser for optical injection unlocking (Chapter 4), and the ADC and FPGA data acquisition electronics replaced the data acquisition card (Chapter 5). Additional polarization optics were also installed, and the etalon was replaced with an OHD system for analyzing the main laser frequency (details in Appendix B).

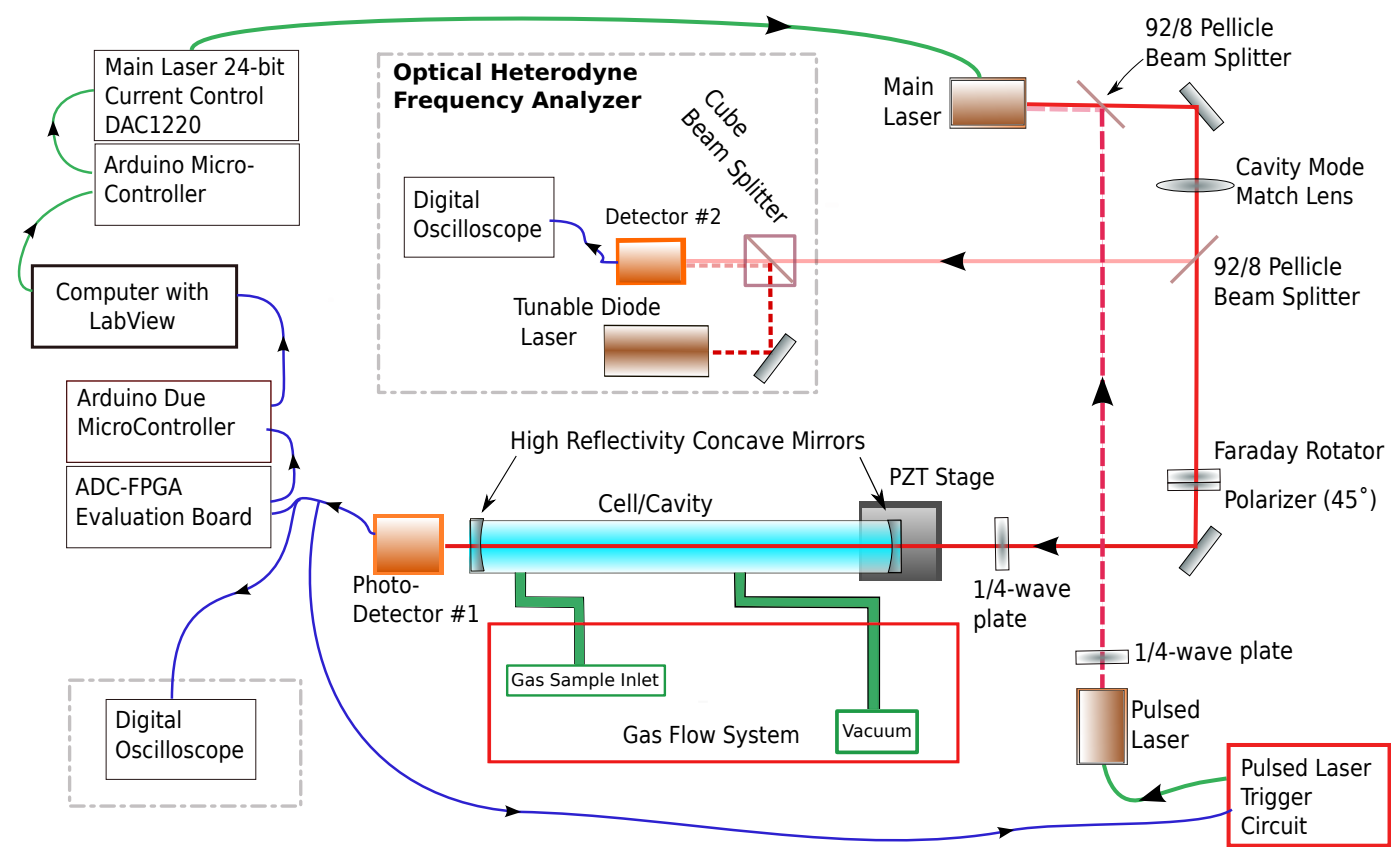

Figure 5 Overall system block diagram. Major components are discussed in the following sections. The main laser is injected into the high-finesse cavity. A pulsed laser induces a frequency shift on the laser once triggered. The cavity output is measured with a photodetector, with the output voltage processed using an ADC-FPGA system. A desktop computer with LabView code controls the entire system. The OHD frequency analyzer system and the digital oscilloscope are ancillary diagnostic tools, not necessary for spectroscopy. 


\section{Chapter 3 Cavity-Laser Locking}

One of the advances that has contributed to higher precision in CRDS instruments is the use of feedback to stabilize the laser frequency on a mode of the cavity ${ }^{23,39-41}$. This enables the build-up of energy in the cavity to occur more efficiently (and therefore more rapidly), allows for faster acquisition of ringdowns, and reduces error in determining the laser frequency by using the equally spaced cavity mode frequencies as a scale. This feedback can be either electronic, typically using the Pound-Drever-Hall method ${ }^{47}$, or optical $^{48,49}$. In the case of optical feedback, it has the added benefit of narrowing the linewidth of the laser. Since the laser becomes more stable and the linewidth narrows, the use of optical feedback reduces the specification requirements on the laser, allowing for the use of inexpensive diode lasers in CRDS systems (lasers chosen in the near-IR are typically for optical communication applications). Since we implemented a form of optical feedback in our design, the theory is briefly discussed in the next section.

\subsection{Coherent Optical Feedback Theory}

Optical feedback involves injecting externally filtered, or simply reflected, laser output back into the lasing medium. This positive feedback, depending especially on the relative intensity of the reflected energy coupled back into the laser, can cause laser frequency stabilization (at low feedback intensities) or induce chaotic behavior (at high feedback levels). Entire books have been written on the study of optical feedback and its effects $^{50,51}$. Conventional optical feedback, the result of the reflection from a single 
external mirror, is described mathematically by the Lang-Kobayashi equations ${ }^{52}$, which are composed of the rate equations for the emitted (complex) electric field and carrierdensity, with an additional term accounting for the time-delayed electric field due to the reflection from an external mirror:

$$
\begin{gathered}
\frac{d}{d t} E(t) e^{i \Omega t}=\left[i \omega_{N}(n)+\frac{1}{2}\left(G(n)-\Gamma_{0}\right)\right] E(t) e^{i \Omega t}+\kappa E(t-\tau) e^{i \Omega(t-\tau)} \\
\frac{d}{d t} n=\frac{J}{e d}-\frac{n}{\tau_{s}}-G(n)|E|^{2}
\end{gathered}
$$

where $E(t)$ is the amplitude of the emitted electric field, $\Omega(t)$ is the phase of the electric field, $n$ is the carrier density, $\omega_{\mathrm{N}}$ and $G$ are the carrier density dependent laser diode resonant frequency and gain, respectively, $\Gamma_{0}$ is the optical loss in the diode cavity, $\kappa$ is the feedback strength, $\tau$ is the round trip delay of the feedback signal, $J$ is the current density, $e$ is the fundamental electric charge, $d$ is the length of the lasing medium and $\tau_{\mathrm{s}}$ is the spontaneous emission lifetime ${ }^{52}$.

If the feedback is from something other than the reflection of an external mirror, then the feedback term in the Lang-Kobayashi equations is simply modified ${ }^{51}$ :

$$
\begin{aligned}
& \frac{d}{d t} E(t)=\frac{1}{2} G_{n}\left\{n(t)-n_{t h}\right\} E(t)+\frac{\kappa}{\tau_{i n}} F(t) \\
& \frac{d}{d t} n(t)=\frac{J}{e d}-\frac{n(t)}{\tau_{s}}-\left.G_{n}\left\{n(t)-n_{t h}\right\} E(t)\right|^{2}
\end{aligned}
$$


where $F(t)$ is the feedback electric field resulting from the transfer function of the external device.

Various optical feedback techniques, each with different forms of the feedback term $F(t)$, are used in the design of stable, tunable, narrow linewidth diode lasers: Extended cavity diode lasers (ECDL) lasers use a single external mirror instead of the laser's output facet and are described by equations (4) and (5); Distributed Feedback (DFB) lasers use a diffraction grating which is part of the laser cavity to provide feedback; and the Littman-Metcalf configuration uses an external diffraction grating and mirror to reflect a high order fringe back into the laser to provide the feedback with high resolution wavelength selection obtained by changing the angle of the mirror ${ }^{53}$. These last two types are forms of filtered optical feedback, described by equations (6a) and (6b).

The method most applicable to CRDS is to use filtered optical feedback from the external high-finesse resonant cavity to provide very narrow bandpass filtering of the laser output. Initial studies on this technique were conducted by Dahmani, et. al. ${ }^{49}$ and Laurent, et.al. ${ }^{48}$ using off-axis confocal resonant cavities, and they showed that under certain conditions, the laser would "lock" to cavity resonance modes, significantly narrowing the linewidth of the laser and stabilizing the frequency to the cavity resonance mode.

For an external high-finesse cavity, the response function is a Lorentzian function and in the time domain has the form:

$$
r(t)=\Lambda \exp \left(-\Lambda|t|-i\left(\omega_{c}-\omega\right) t\right)
$$


where $\Lambda$ is the half-width half-maximum of the filter, and $\omega_{c}$ is the center of the filter. The feedback term becomes the convolution of the input and the response ${ }^{51}$ :

$$
F(t)=\int_{-\infty}^{t} r\left(t^{\prime}-t\right) E\left(t^{\prime}\right) d t^{\prime}
$$

While the output of the cavity is narrowly filtered around the cavity mode, there is a strong reflection off the first cavity mirror back towards the laser ( $\sim 99 \%$ of incident power for high-finesse cavity mirrors) that is not filtered by the cavity. To avoid feedback effects from this light, as in the case of Dahmani, et.al. ${ }^{49}$ and Laurent, et.al. ${ }^{48}$, the input beam was injected slightly off-axis, directing this strong reflection away from the laser while still allowing light exiting the cavity to follow the path back to the laser. This results in slightly divergent spots at the other end of a two-mirror cavity, which significantly reduces the cavity finesse (and thus ringdown time).

Since the optical feedback described above is provided by a high-finesse cavity, it was then applied to cavity-based spectroscopies (e.g., CRDS and ICOS). However, a disadvantage to the off-axis confocal design mentioned above $e^{48,49}$ is that the finesse of the cavity is highly degraded due to the off-axis injection, making it undesirable for CRDS since high cavity finesse implies smaller background decay constants $\beta_{0}$ (corresponding to longer decay times and better base sensitivity in spectroscopy). To take advantage of the optical feedback, while maintaining the high finesse of the cavity, and still diverting the direct reflection from the first cavity mirror, this locking effect was later revised for CRDS using a 3-mirror V-cavity by Morville et.al. ${ }^{54}$. An alternate setup which removed 
the strong feedback from a direct reflection by inserting a glass plate inserted inside the cavity at the Brewster angle was demonstrated by the same group ${ }^{41}$. A small amount of power is injected into the cavity off of the plate, and only the significant buildup in intracavity intensity when the frequency matched a cavity mode provided a significant amount of feedback to the laser, but this still significantly lowered the finesse of this system. For our configuration, we implemented a simpler linear 2-mirror cavity design, which is described in Section 3.2.

Examination of the effect of optical feedback is typically accomplished by analyzing the laser output frequency. The relationship between the free-running laser frequency and the frequency with feedback is taken from Laurent et.al. ${ }^{48}$ :

$$
\begin{aligned}
\omega_{\text {free }} & =\omega+K \frac{\sin \left(\frac{2 \omega}{c}\left(L_{d}+L_{c}\right)+\theta\right)-r^{4} \sin \left(\frac{2 \omega}{c}\left(L_{d}-L_{p}\right)+\theta\right)}{1+\frac{4 F_{c}^{2}}{\pi^{2}} \sin ^{2}\left(\frac{2 \omega}{c} L_{p}\right)} \\
K & =\frac{1}{2} \sqrt{\beta\left(1+\alpha^{2}\right)} \frac{c}{2 \eta l_{d}} \frac{F_{c}}{F_{d}}
\end{aligned}
$$

where $\omega_{\text {free }}$ is the laser free-running frequency, $\omega$ is the laser-frequency with feedback, $F_{c}$ is the finesse of the cavity, $F_{d}$ is the finesse of the diode laser, $\alpha$ is the linewidth enhancement factor for the laser, $L_{p}$, and $L_{d}$, are the cavity length and laser-cavity distance, respectively, $r$ is the reflectivity of the cavity mirrors, $c$ is the speed of light, and $\theta=\tan ^{-1}(\alpha)$, and $\beta$ is the proportion of the laser power received as optical feedback (which, for stabilization is typically a constant in the range $10^{-3}$ to $10^{-6}$, and the value of $K$ is then typically $\sim 10^{9}$ to $10^{12}$ ). When the distance between the laser and the cavity is a 
multiple of the cavity length, the feedback will stabilize the laser to the cavity mode frequency. Taking the simple case when $L_{p}=L_{d}$, the equation simplifies to:

$$
\omega_{\text {free }}=\omega+K \frac{\sin \left(\frac{2 \omega}{c} L_{c}\right)}{1+\frac{4 F_{c}^{2}}{\pi^{2}} \sin ^{2}\left(\frac{2 \omega}{c} L_{c}\right)}
$$

To estimate the performance for our setup, we used $F_{\mathrm{c}}=10,000$, and estimated the following: $\beta=1 \times 10^{-5}, F_{\mathrm{d}}=3, \alpha=4, \eta=3.5$, and $l_{\mathrm{d}}=1 \mathrm{~mm}$, which gives $K \approx 1 \mathrm{x}$ $10^{11}$, and a plot relating $\omega_{\text {free }}$ and $\omega$ is shown in Figure 6 . The shallow slope around $\omega_{\text {res }}$ indicates the locking effect of the feedback, as the free-running laser would normally deviate far from the center of the cavity mode, while the coupled output frequency is orders of magnitude lower ${ }^{54}$. Once the locking range is exceeded, there is a jump in the coupled frequency and if the frequency continues to increase, it will approach (and then lock to) the next cavity mode. In the figure, the estimated locking range of our optical feedback is $8 \mathrm{MHz}$, and the slope is $120 \mathrm{rad} / \mathrm{rad}$. 


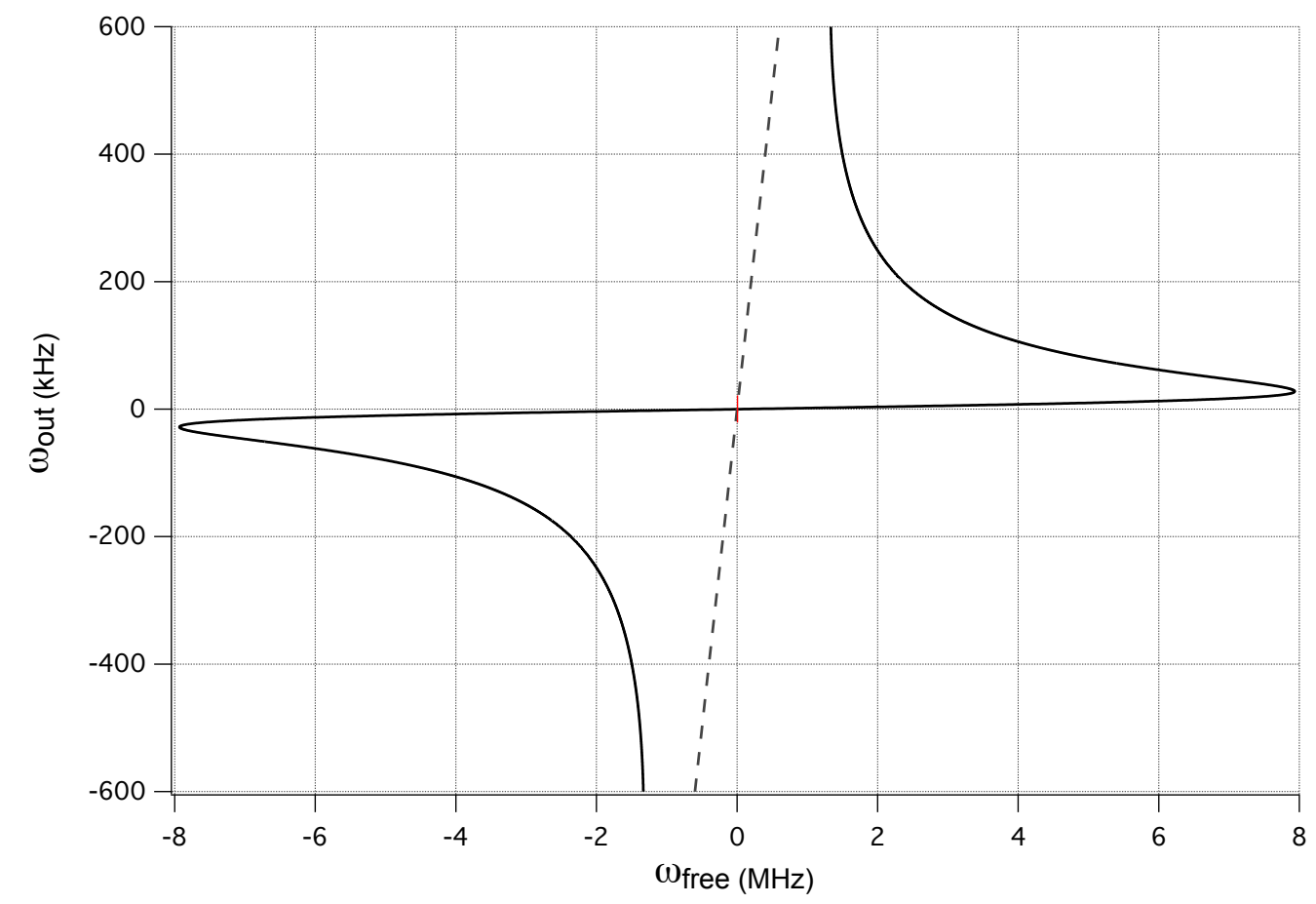

Figure 6 Effect of optical feedback on laser output. The dashed line shows the laser output frequency if there were no optical feedback (i.e., it is a 1:1 line). The solid line shows the output frequency in the presence of feedback, and the shallow slope around 0 (which represents a mode of the cavity) indicates the frequency locking - the laser output does not move far from resonance. In this case, the locking range would be $\pm 8.0 \mathrm{MHz}$ (the extremes of $\omega_{\text {free }}$ ) and the slope is $120 \mathrm{rad} / \mathrm{rad}$.

A drawback of coherent optical feedback is that the locking performance is highly dependent on the phase of the feedback signal when it reaches the lasing medium because the emitted and feedback electric fields add coherently. Therefore the laser-cavity distance must be kept stable, and if sweeping over many cavity modes to obtain a spectrum, the phase change must be compensated for by actively adjusting this distance to optimize the feedback level at the laser for each mode ${ }^{54}$.

Coherent optical feedback techniques enable fast data acquisition since neither the cavity nor the laser needs to be dithered to achieve an overlap of the laser and the cavity 
(buildup and ringdown). Although they did not use CRDS, Morville et.al. reported sweeping 200 modes in $100 \mathrm{~ms}$ with active phase control enabled, to obtain a gas-phase $\mathrm{H}_{2} \mathrm{O}$ spectra $^{54}$. Similarly Motto-Ros et. al. used CRDS and were able to sweep 90 cavity modes in $140 \mathrm{~ms}$, and obtain a 50 point average of each mode in 7 seconds $^{41}$.

\subsection{Linear Optical Feedback Setup}

In our current system, we implemented a simplified approach to laser-cavity locking. Since the requirement for cavity locking using coherent feedback is that the ratio of laser-cavity distance to cavity length be an integer, rather than using a V-cavity or offaxis injection, we chose to use linear cavity feedback design (see Figure 5). In this configuration, both the direct reflection from the first cavity mirror and the output from the cavity contributed to the feedback entering the laser medium. (Note that the cavity output - the ringdown signal — propagates in both directions along the cavity axis, although only the direction toward the photodiode is used to measure the ringdown.) By adjusting the distance from the laser to the cavity to be approximately equal to that of the cavity length, laser-cavity interaction was enhanced at the mode of either, since they approximately overlapped. Moreover the laser-cavity path is not a high-finesse path, so its broad linewidth enabled sufficient overlap that the laser stabilized to the cavity mode even when the two distances are not exactly the same.

To demonstrate the effect of cavity locking in our system, we measured the linewidth of the laser while it was injecting light into the cavity, using the OHD frequency analysis system described in Appendix B. This system was capable of measuring the main laser's frequency relative to a stable reference laser (in this case it is 
the New Focus ECDL used in our previous setup described in Section 1.3). The main drawback was that the difference between the two lasers' frequencies must be within the bandwidth of the photodetector-for the Thorlabs PDA255, the bandwidth is $65 \mathrm{MHz}$. This required tuning of the ECDL until it was close enough to the main laser.

In Figure 7, the OHD frequency spectrum of the main laser is shown for two cases. In the first (in blue), the system was in the typical configuration with the cavity fully aligned for spectroscopic measurements, so both the cavity and the laser-cavity path are providing feedback to the laser. The second (in red) was with the second CRDS cavity mirror misaligned. This means there was no feedback from the cavity itself, only direct reflection from the first mirror, and feedback due only to the laser-cavity path. As the figure shows, there was a significant linewidth narrowing when the cavity was aligned, meaning that the optical feedback was having an effect on the main laser.

To confirm that the main laser was locked to the cavity mode, we looked at the cavity output in the time domain, and noted that the output level built up to a significant voltage and stayed high. Figure 8 shows the output voltage of the cavity photodetector with optical feedback for the laser. The dropouts were triggered by our pulse circuit (see Chapter 4) and the envelope of the output voltage demonstrates the stability of the laser. Without locking, cavity buildup would last less than $100 \mu \mathrm{s}$, and there would be a significant time before cavity buildup restarted. 


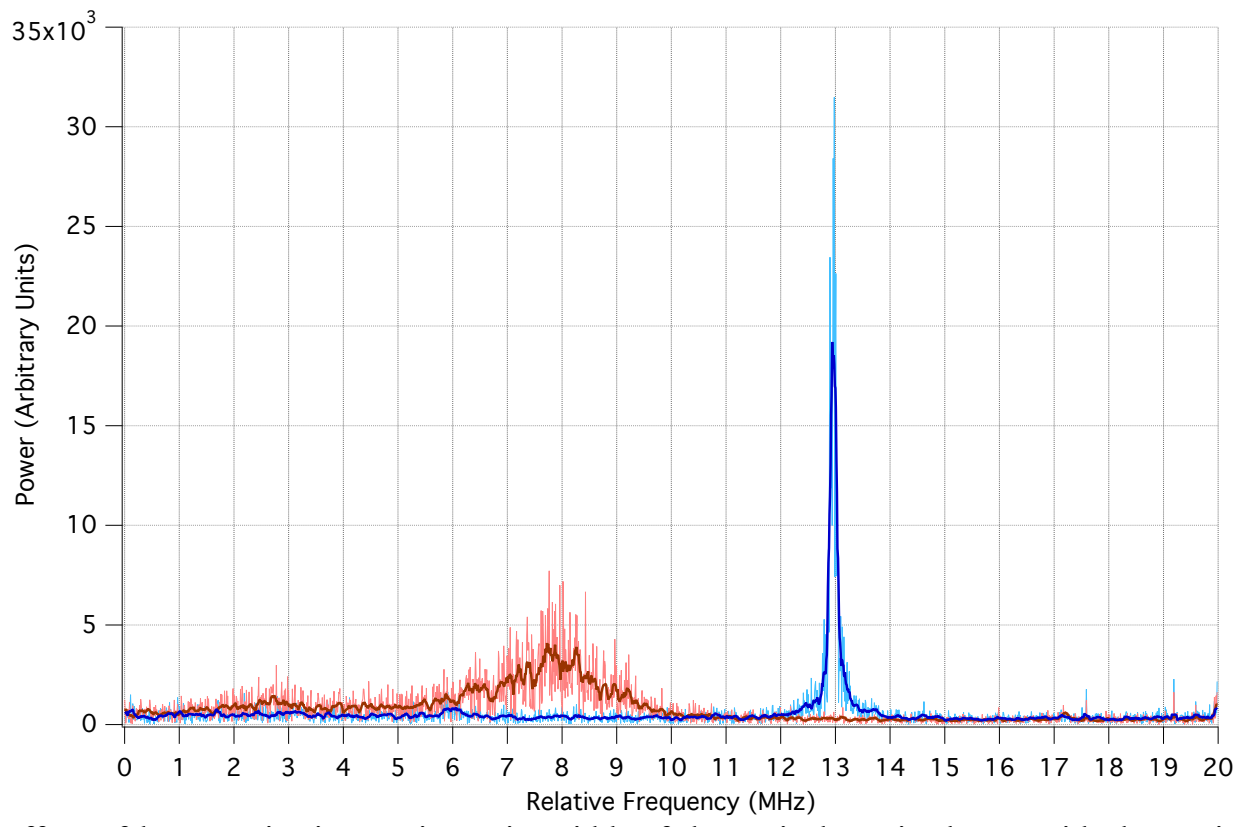

Figure 7 Effect of laser-cavity interaction. Linewidth of the main laser is shown with the cavity aligned (blue) and then when the cavity output mirror is mis-aligned (red). The main laser linewidth is significantly narrowed when the cavity output is included in the feedback. A 10-point moving average is shown on the misaligned cavity to better represent the peak.

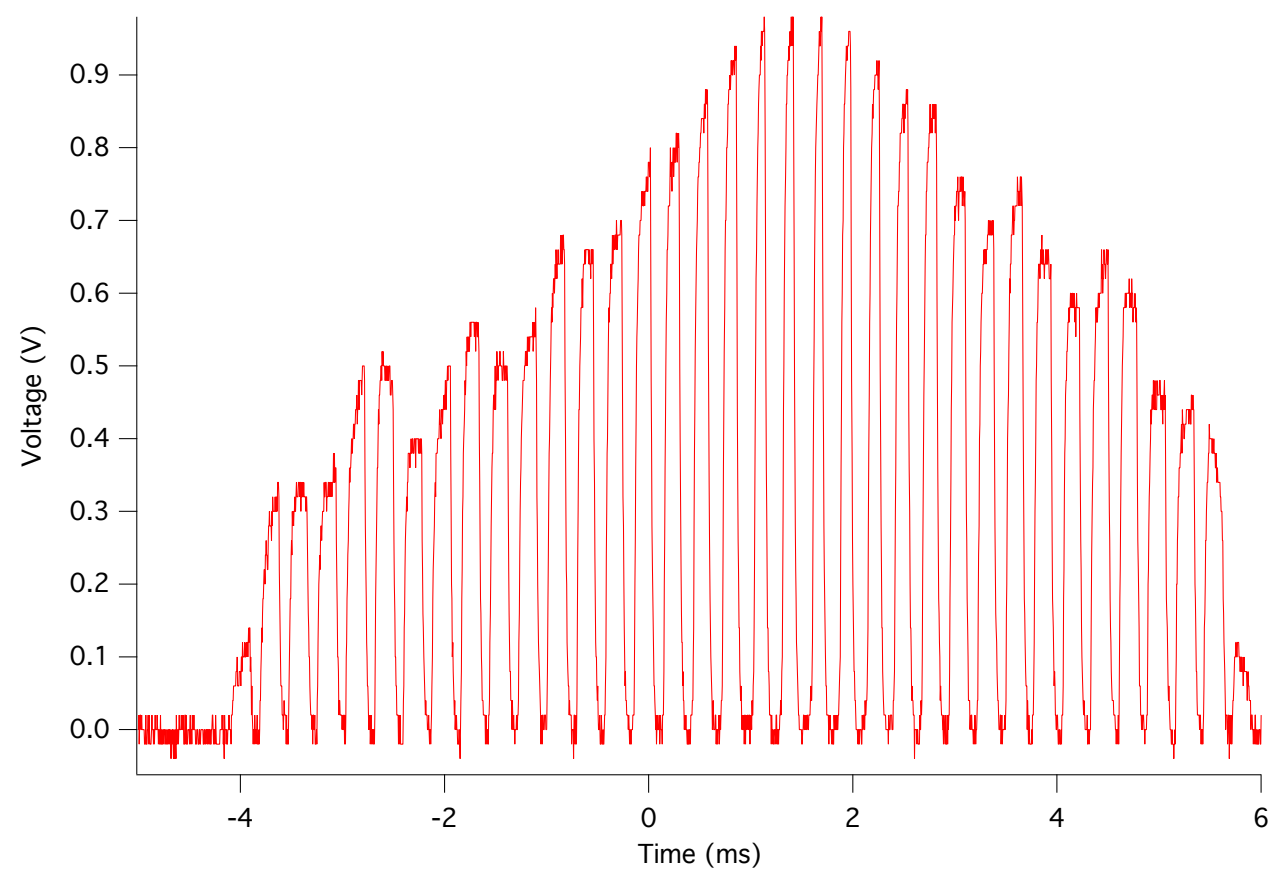

Figure 8 Extended Laser-Cavity interaction with induced ringdowns. The long-term increase (and then decrease) in peak voltage demonstrates that the laser frequency remained close to the cavity mode for extended periods of time (10 ms in this diagram). The drop outs were ringdowns induced by the pulsed laser, as described in Section 2.4. Without locking the build-up would only exist for on the order of $100 \mu \mathrm{s}$. 
The cavity locking enabled light build-ups (and subsequent ringdowns) to occur at a significantly higher rate of up to $3.5 \mathrm{kHz}$, compared to the $10-\mathrm{Hz}$ rate achieved with the cavity length dithering method employed previously. The ringdown portion of the cycle was limited by the length of time data was collected for each event, so it would be possible for the optical part of the system to have a higher ringdown acquisition rate if the decay constant were larger, but obviously would degrade the sensitivity of the spectroscopic measurement. Further discussion of the optical limitations to the ringdown rate are discussed in Section 4.4.

\subsection{Laser Frequency Control}

Having implemented a method to lock the laser to a cavity mode, it was then necessary to adjust the laser frequency from one mode to the next, in order to measure the absorption as a function of frequency (or more typically, wavenumber in units $\mathrm{of}^{-1}$ ) to obtain a spectrum for analysis. For our distributed feedback DFB diode laser, the frequency could be varied in two ways. First, adjusting the operating temperature of the laser coarsely tuned the frequency; and, for this laser we empirically determined the wavenumber as a function of temperature using the OHD technique (See Appendix B). The results for 3 trials are shown in Figure 9, and we determined that the wavenumber changed by $-0.55 \mathrm{~cm}^{-1} /{ }^{\circ} \mathrm{C}$ ( or $+0.093 \mathrm{~nm} /{ }^{\circ} \mathrm{C}$ ), which is the average of the three linear fits shown. 


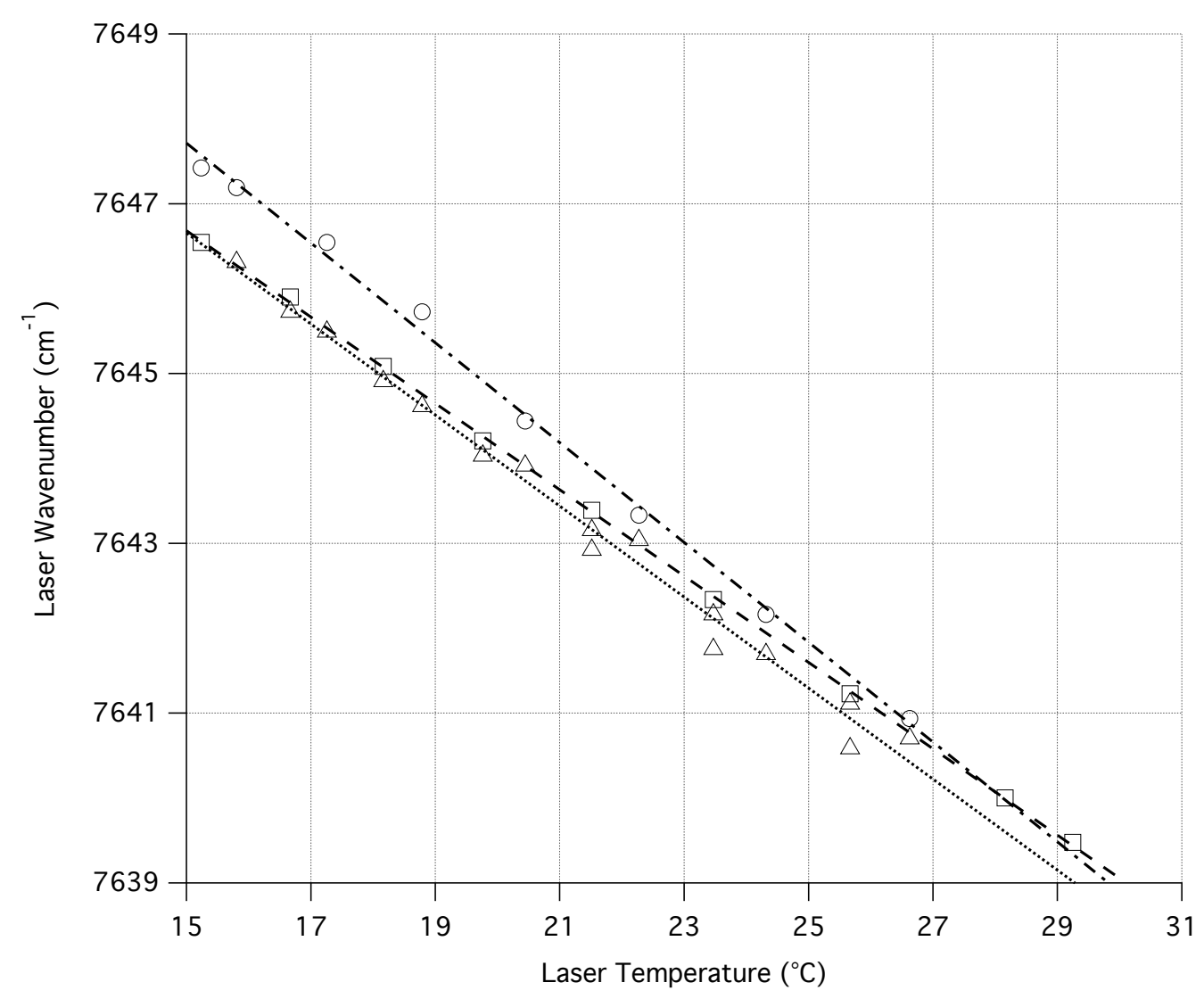

Figure 9 Plot of data taken for wavelength vs. Temperature for the Main Laser. Three datasets were taken on three different days. The average slope for these three days is $-0.55 \mathrm{~cm}^{-1} /{ }^{\circ} \mathrm{C}$.

High-resolution spectroscopy requires scanning across absorption peaks with linewidths on the order of $0.1 \mathrm{~cm}^{-1}$ or less, so that finer tuning of the laser wavenumber is required, and this tuning was accomplished by adjusting the laser current. Again, using the OHD technique (See Appendix B), it was determined that the wavenumber changed at a rate of $0.035 \mathrm{~cm}^{-1} / \mathrm{mA}(1.06 \mathrm{GHz} / \mathrm{mA})$. Unfortunately, the laser current also determines the power, with a typical slope efficiency of $0.5 \mathrm{~mW} / \mathrm{mA}$, so a $1 \mathrm{~cm}^{-1}$ change corresponded to a $14 \mathrm{~mW}$ change in laser output power.

The laser current was adjusted through an applied voltage input (-5 to $5 \mathrm{~V}$ ) on the laser controller (Model ITC102, Thorlabs, Inc., Newton, New Jersey) at a conversion rate 
of $40 \mathrm{~mA} / \mathrm{V}$. The laser controller's current control input has an impedance of $10 \mathrm{k} \Omega$. This translates to $1.4 \mathrm{~cm}^{-1} / \mathrm{V}$ (or $42.4 \mathrm{MHz} / \mathrm{mV}$ ). Achieving $1 \mathrm{MHz}$ resolution (more than sufficient if the locking range is expected to be $8 \mathrm{MHz}$ ) for frequency control requires $\sim 24 \mu \mathrm{V}$ resolution in the applied voltage. Therefore a computer-controlled high-precision DAC voltage supply was necessary. We chose an inexpensive evaluation board (\$49) with a 20-bit DAC (DAC1220EVM, Texas Instruments, Dallas, Texas) that provided a single-ended $0-5 \mathrm{~V}$ output range, with a minimum resolution of $5 \mu \mathrm{V}^{55}$. Since noise in the output voltage causes frequency fluctuations at the laser, a low-ripple, low-noise linear power supply was used for the evaluation board so that the EMI found in typical switching power supplies would not pose an issue. To further eliminate power supply or digital noise on the DAC output, an RC low-pass filter (cutoff frequency of $23 \mathrm{~Hz}$ ) and a voltage divider were added to the output of the evaluation board, as shown in Figure 10.

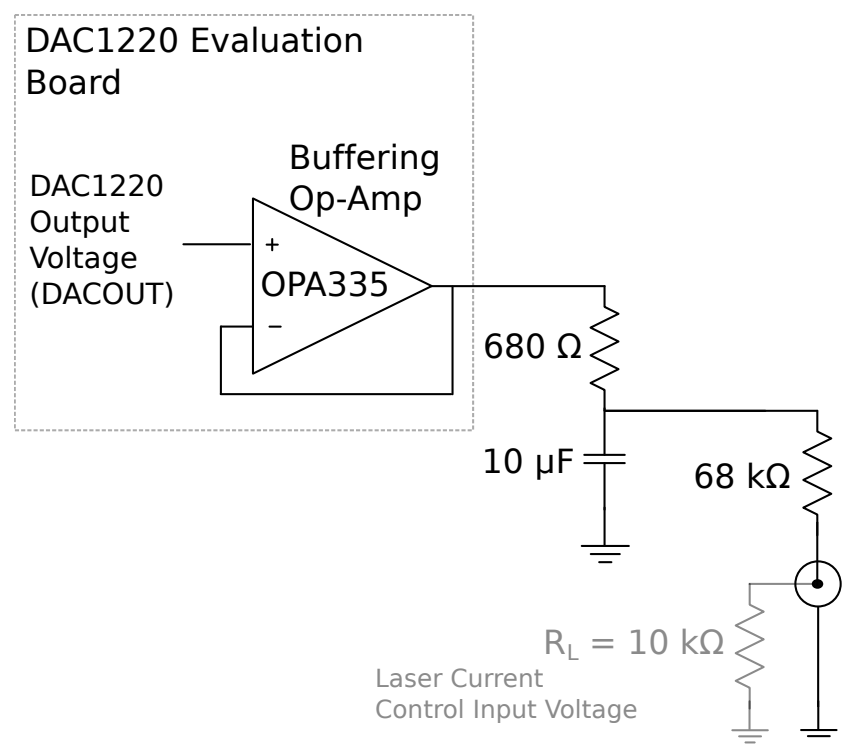

Figure 10 Circuit Diagram for DAC 1220 output for filtering and attenuating the signal. The buffered output of the DAC1220 evaluation board was passed through a RC low-pass filter and a voltage divider. 
With this circuit, a DAC output of 0-3 V corresponded to an input voltage range of 0-0.667 V, a current range of $15 \mathrm{~mA}$, and a wavenumber range of $0.525 \mathrm{~cm}^{-1}(16 \mathrm{GHz})$. Using this relationship between the DAC output voltage and the laser current, combined with the data collected for laser wavenumber as a function of current, we obtained a function relating the laser wavenumber to the DAC output voltage. A plot, including the curve fit, is shown in Figure 11, and the curve fit enabled determination of the absorption spectrum using the set voltage of the DAC and the best-fit curve equation. This function was updated (see Section 6.3) when we compared our obtained spectra with the theoretical spectra at that pressure.

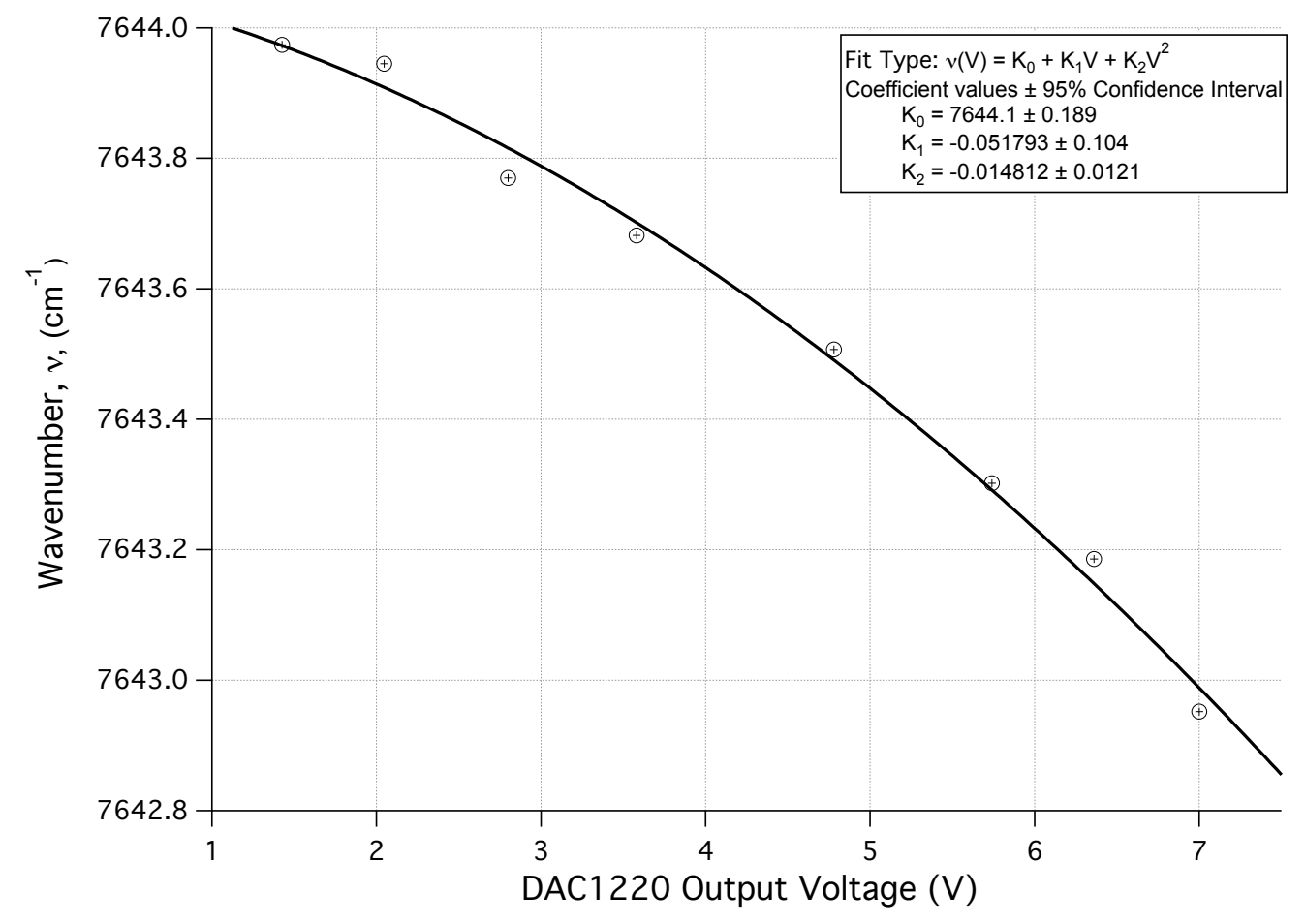

Figure 11 Main Laser Wavenumber as a function of current-control applied voltage. The quadratic fit parameters are shown, along with the $95 \%$ confidence interval for those values. An improved fit for this is given in Section 6.3, based on comparison with a published spectrum. 
The DAC1220 was controlled using the 3-wire Serial Peripheral Interface (SPI) standard ${ }^{55}$, which required an additional interface between the evaluation board and the computer for control. An Arduino Uno microcontroller (Arduino, SA., Turin, Italy), with a built-in SPI interface that communicates with a computer via USB, was programmed to take a command from the computer and output the proper 16-bit command code and the 20-bit binary value (as two additional 16-bit integers) to the DAC1220 evaluation board. The code for the Arduino Uno that controlled the DAC1220 is provided in Appendix E. 


\section{Chapter 4 Optical Frequency Unlocking ${ }^{1}$}

Cavity Ring-down Spectroscopy (CRDS) is a sensitive optical extinction technique that uses the exponential decay of light energy from a high-finesse optical cavity to determine the total losses in the cavity. In order to initiate the decay of light out of the cavity, the injection of light into the cavity must be stopped on short timescales (usually $<1 \mu \mathrm{s})$. Typically this is accomplished by interrupting the laser with an external optical switch, such as an acousto-optic modulator or electro-optic modulator ${ }^{17,39,56}$. These devices are costly $(\$ 2000-\$ 7000)$, especially in the near-IR range. Additionally, the acousto-optic modulator introduces a Doppler frequency shift, and can interfere with optical feedback techniques used to lock the laser to the cavity ${ }^{54}$. Electro-optic modulators use electrically controlled polarization switching which does not shift frequency, and therefore is a common choice for pulsing the laser ${ }^{57}$. The requirement for switching hundreds to thousands of volts over times of 10-100 ns complicates the design of these devices. A mechanical method of shifting the cavity off-resonance by mounting one of the cavity mirrors on a piezoelectric transducer and applying a voltage was demonstrated by Hahn, et.al. ${ }^{58}$, but this method could be problematic for systems that employ optical feedback to lock the laser to the cavity since the cavity length is continuously changing. Another technique to modulate the laser intensity is to simply

\footnotetext{
${ }^{1}$ Most of this chapter is taken from a paper published in Optics Letters entitled "Optical Frequency Unlocking for Cavity Ringdown Spectroscopy"46, and is reproduced here with permission from the OSA. The paper can be found at the following URL on the OSA website: http://dx.doi.org/10.1364/OL.39.004227. Systematic or multiple reproduction or distribution to multiple locations via electronic or other means is prohibited and is subject to penalties under law.
} 
pulse the laser drive current ${ }^{41}$; but, with this technique the laser driver bandwidth limits the time the laser can be kept off, and the current modulation induces thermal frequency shifts. These issues increase the complexity in the fitting algorithm, the current pulsing timing, and the frequency shifting circuit. Using opto-electronic locking to the cavity, such as the Pound-Drever-Hall method ${ }^{47}$, requires additional circuit complexity to turn off the electronic locking during the ringdown, and then re-lock the laser to the cavity ${ }^{58-}$ 60

In this chapter we present a novel technique to move the ring-down laser off resonance in less than a microsecond, while not interfering with cavity locking, by injecting a second, pulsed diode laser into the main laser. This method does not change the current of the locked laser, allowing it to return to cavity resonance immediately after the disrupting pulse is over. It is also inexpensive and requires only a diode laser, a beam splitter, and a simple pulsing circuit. We first present the method and experimental setup used to demonstrate the technique. Second, we analyze the dependence of the frequency shift on both main laser power and wavelength difference between the pulse laser and main laser. Finally, we demonstrate cavity ringdown using this technique.

\subsection{Optical Injection}

A diode laser is susceptible to the introduction of external electromagnetic fields, resulting in a continuum of effects, from chaotic frequency instabilities to frequency stabilization. These effects have been studied fairly extensively ${ }^{51}$. The delayed reinjection of light produced by a laser and spectrally filtered by a cavity has been used to lock the diode laser to the cavity for optical feedback cavity-enhanced spectroscopy ${ }^{48}$. The effect 
of optical injection on a diode laser was included in a review article by Tarjwik and Lenstra $^{61}$. The typical application of optical injection is to stabilize and lock a slave laser to a master laser, by influencing the refractive index and the electric field inside the slave laser's medium. The change in refractive index directly causes a shift in the lasing frequency (since the frequency is determined by the speed of light in the medium and the cavity length). The locking of the slave laser requires that the master and slave laser be close (typically within a few $\mathrm{GHz}$ ). However, the refractive index changes (which in turn alter the resonance frequency of the laser cavity) occur over a much wider range, as long as the energy of the photon induces changes in carrier density. The effect of changes in carrier density on the laser materials has been studied by others: see Figure 3 in Bennett et.al. ${ }^{62}$, equation 4 in Kowalsky and Ebeling ${ }^{63}$, and Ishida et.al. ${ }^{64}$ It is this effect that we exploit to shift the frequency in this work.

\subsection{System Configuration}

The experimental setup is shown in Figure 12. A distributed feedback laser (Mitsubishi, ML725B11F, with an approximate wavelength of $1308 \mathrm{~nm}$ ) to be used for spectroscopy (as the main ring-down laser) is directed into a near-confocal high-finesse optical cavity comprised of two plano-concave mirrors (Newport, \#10CV00SR.60F, Reflectivity $>99.97 \%$, yielding a cavity finesse of $>10000$ ). A $92 \% / 8 \%$ beamsplitter directs a small fraction of a pulsed Fabry-Perot laser (Mitsubishi, ML725B8F, with an approximate wavelength of $1309 \mathrm{~nm}$ ) into the main laser, while allowing a large fraction of the main laser to pass to the cavity. A second beamsplitter samples the main laser and sends it into an OHD frequency analyzer (see Appendix B). This analyzer uses a stable 
tunable diode laser (New Focus, Model 6324, with a linewidth of $300 \mathrm{kHz}$ in a $50 \mathrm{~ms}$ integration time, and a drift of $<5 \mathrm{MHz}$ over $1 \mathrm{~s}$ ) as a reference that mixes with a laser under test on the photodetector (Thorlabs, Model PDA255). The mixing produces a beat frequency that indicates the frequency of the laser under test relative to the reference laser. The photodetector signal output is connected to a digital oscilloscope (Tektronix Model TDS-3052) to collect the voltage versus time for frequency analysis using Fast Fourier Transform (FFT). As long as the beat frequency is within the bandwidth of the photodetector (approximately $100 \mathrm{MHz}$ ), this accurately measures the frequency shift induced by the pulsed laser. The OHD system is only used for experimental characterization and would not be part of an eventual implementation of this technique. When the main laser is unlocked from the cavity, a second photodetector (New Focus, Model 1811) at the output of the cavity allows measurement of the exponential decay of the cavity energy (the ringdown) using the digital oscilloscope triggered by the pulsing circuitry. 


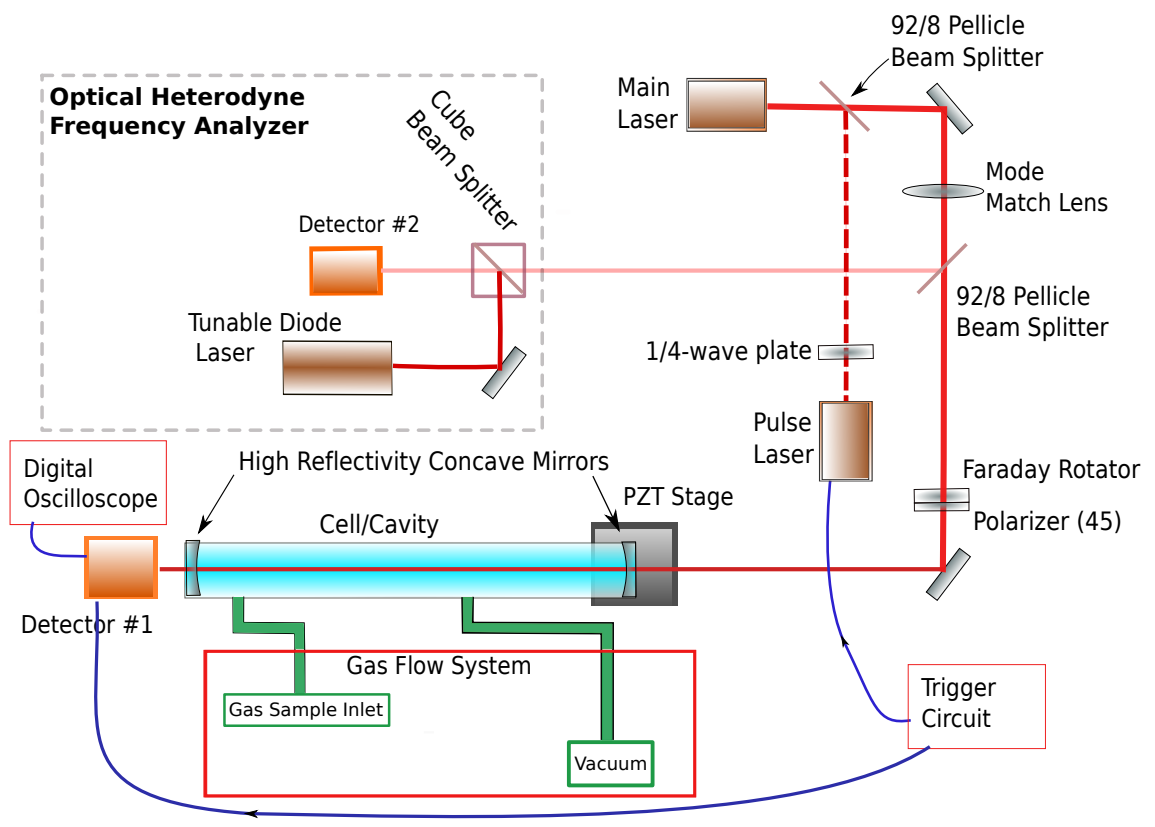

Figure 12 Diagram of the system. The main laser is injected into the optical cavity for the ring-down measurement. A pulsed laser is aligned to inject light back into the main laser. A simple pulsing/triggering circuit is used to activate the pulsed laser. A tunable diode laser and a secondary detector are used to detect frequency shifts in the main laser using OHD for initial characterization (dash-boxed region is not a permanent part of the system). A digital oscilloscope measures the cavity output and optical heterodyne signals. The faraday rotator and polarizer control optical feedback to the main laser to enable locking to the cavity. The quarter-wave plate reduces coherent reflections from the pulsed laser.

The pulsed laser is controlled by a simple multivibrator (timing) circuit based on the standard $555 \mathrm{IC}^{65,66}$. The circuit can be configured as either astable (continuously pulses the laser) or monostable (an external TTL signal triggers a single laser pulse). For testing and measurement of the frequency shift, we configured the circuit in astable mode. For cavity ring-down measurements, the circuit was configured in monostable mode, triggered by a threshold circuit when the cavity output photodetector exceeded a preset voltage. A circuit diagram is shown in Figure 13. Two resistors and a capacitor set 
the nominal $90 \mu$ s pulse width (the length of time the main laser was moved off resonance to observe the ring-down decay). The output voltage from the 555 typically was between $3.5 \mathrm{~V}$ and $3.75 \mathrm{~V}$, which drove the pulsed laser at an estimated power of 2.5 $\mathrm{mW}$ (barely above the lasing threshold current of $5 \mathrm{~mA}$ ). Alignment and wavelength measurements required higher power, so the laser power was increased to approximately $17 \mathrm{~mW}$ (at a drive current of $35 \mathrm{~mA}$ ).

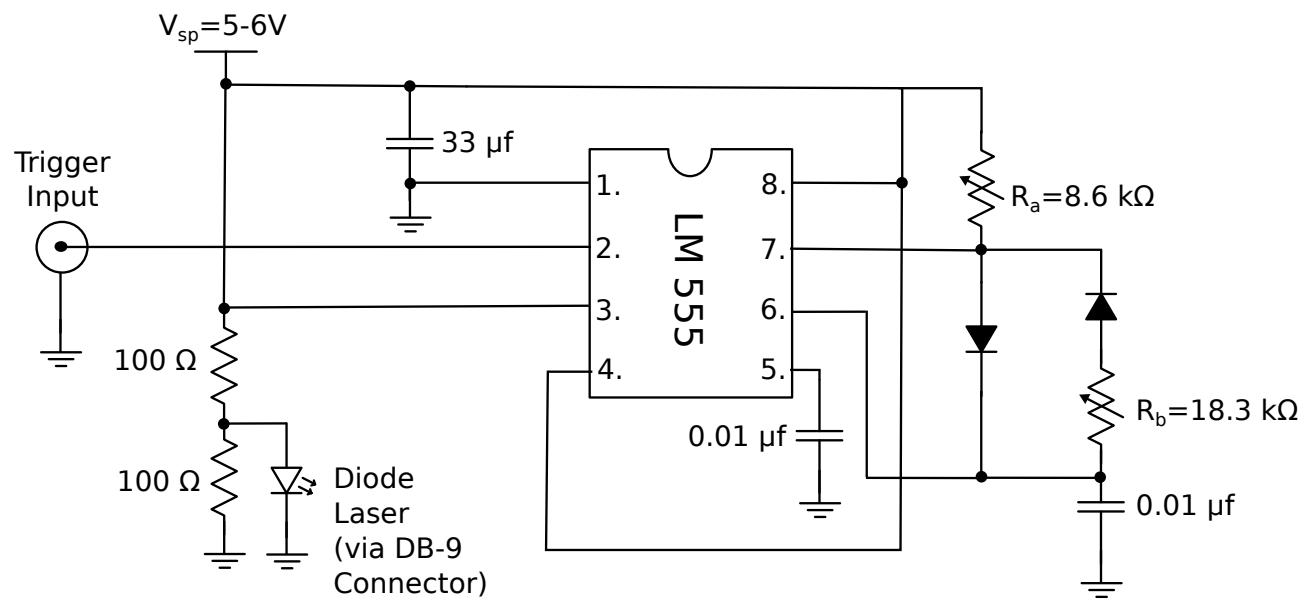

Figure 13 Circuit diagram for pulsed laser. The circuit is an astable multivibrator circuit using a 555 timer IC. Pulse width and duty cycle (in monostable mode) are controlled by adjusting $R a$ and $R_{b}$. The values in the diagram correspond to $90 \mu \mathrm{s}$.

FFT analysis of a typical OHD signal (Figure 14) showed the main laser's peak frequency shifted approximately $20 \mathrm{MHz}$, and returned to the original frequency after the pulse. Time domain analysis showed that this frequency shift occurred within 200-500 ns. 


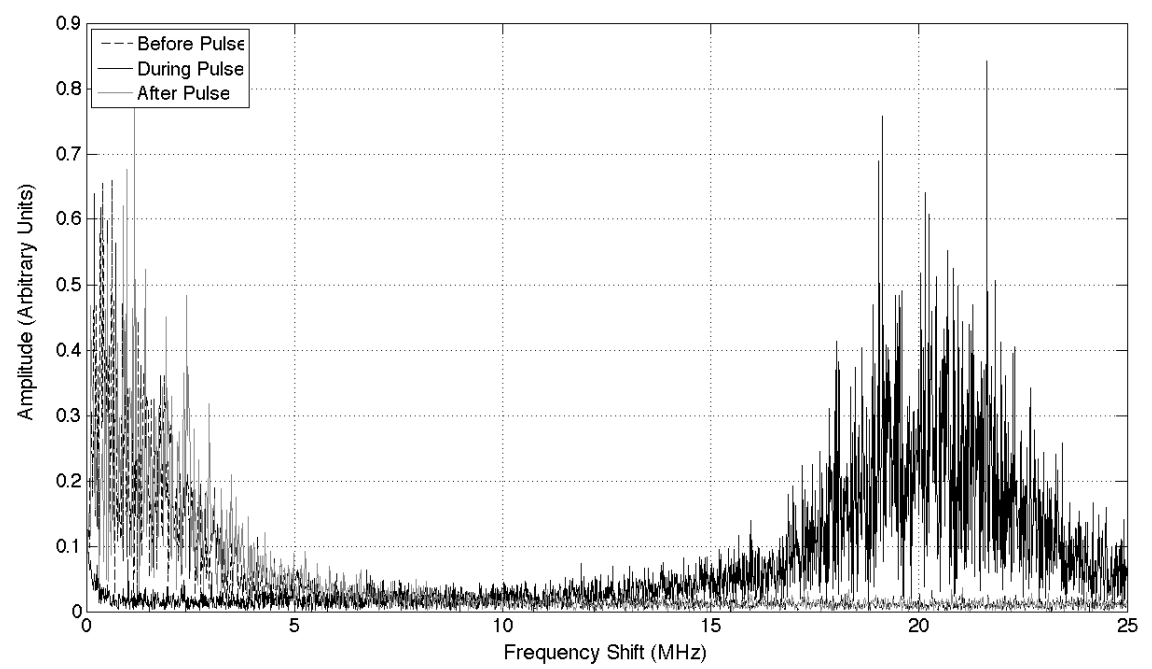

Figure 14 Frequency shift from optical injection unlocking. Three datasets are shown. The FFT of the OHD signal of the laser before (dashed), during (solid black), and after (solid gray). The peak signal before and after overlap, when the pulsed laser is on, the frequency is shifted $20 \mathrm{MHz}$.

\subsection{Frequency Shift Performance (Frequency Domain)}

Measuring a spectrum would require tuning the frequency of the main ring-down laser, presumably without changing the pulsed laser's output, resulting in changes to the frequency difference between the lasers. To examine the frequency shift of the main laser as a function of the frequency difference between the main and pulsed laser, we tuned the main laser over a 1-nm range by varying its operating temperature between approximately $15^{\circ} \mathrm{C}$ and $27^{\circ} \mathrm{C}$. The main laser current was fixed at $10.1 \mathrm{~mA}$, about twice the threshold current. Figure 15 shows the results: a variation in frequency shift between 20 and $100 \mathrm{MHz}$ when the pulsed laser is $>0.1 \mathrm{~nm}$ from the main laser. When the pulsed laser is $<0.1 \mathrm{~nm}$ from the main laser, injection-locking effects dominate, rather than changes in refractive index, and the frequency shift was only about $5 \mathrm{MHz}$. Notably the peak frequency shift occurs when the lasers differ by $0.4 \mathrm{~nm}(70 \mathrm{GHz})$. The explanation 
for this may be related to the peak refractive index changes described in Van Tartwijk et. al. ${ }^{67}$ and Bennett et.al. ${ }^{62}$

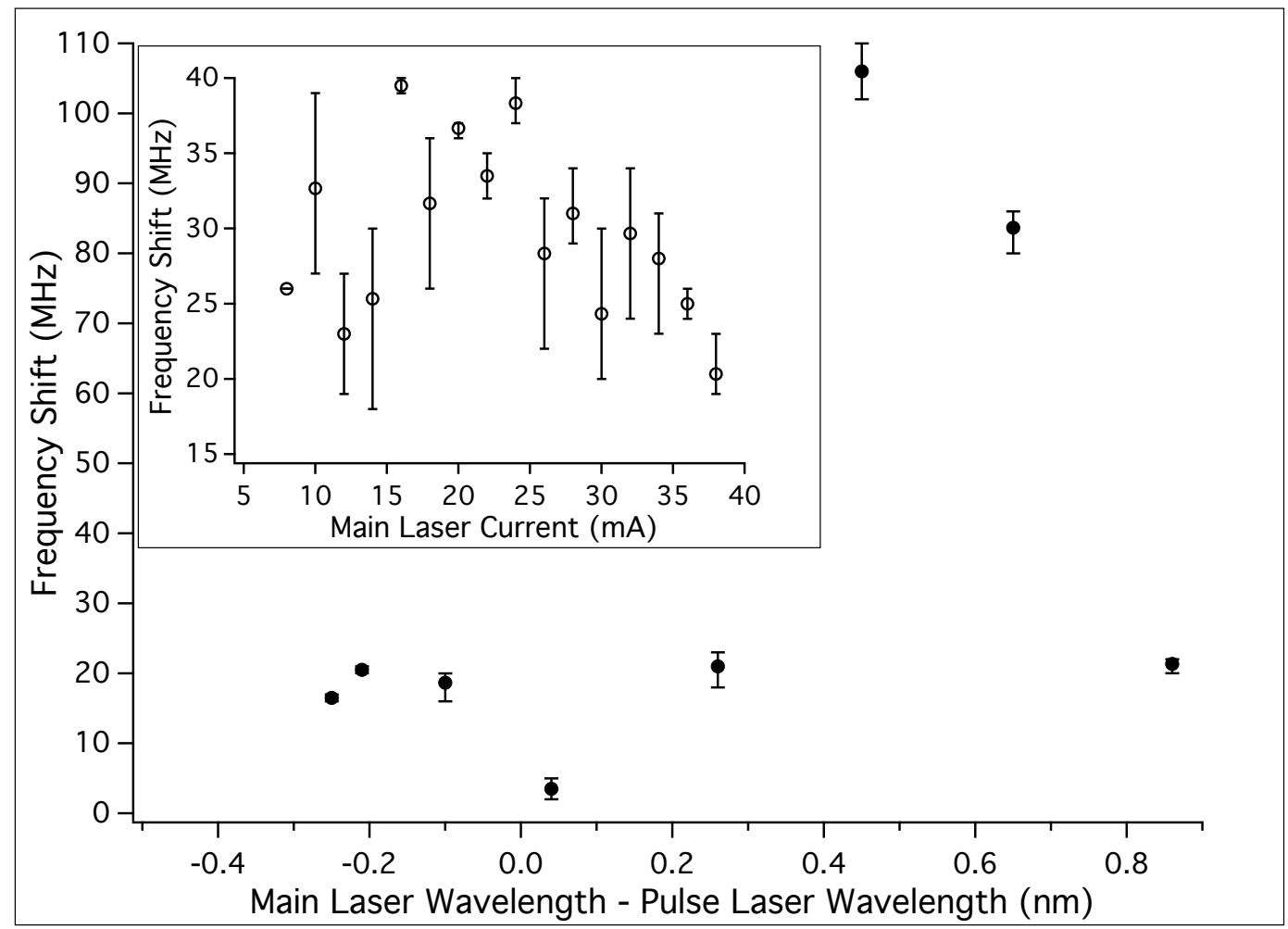

Figure 15 Induced frequency shift as a function of the wavelength difference between the Main Laser and the Pulse Laser. In the main figure, the Main Laser wavelength is adjusted by changing its temperature. The average of 3 different measurements taken at each wavelength is shown, with error bars indicating the max and min. Inset: The frequency shift as a function of Main Laser current, with laser temperature fixed. The range covers approximately $34 \mathrm{GHz}(0.2 \mathrm{~nm})$. Again, average is shown for 3 different measurements at each current level, error bars indicate max and min.

For high-resolution spectroscopy, frequency tuning of the main laser is best accomplished by changing the drive current. For the main laser, the frequency change with current was $1.06 \mathrm{GHz} / \mathrm{mA}$. In the inset of Figure 15, we show the main laser frequency shift as a function of drive current. The main laser was initially tuned to $1308.27 \mathrm{~nm}$; the pulsed laser had a measured wavelength of $1309.45 \mathrm{~nm}$. The $32-\mathrm{mA}$ current range in the inset of Figure 15 represents a frequency range of approximately 34 
GHz. The average shift induced by the pulsed laser was $30 \mathrm{MHz}$ (range 15-40 MHz). For a high-finesse cavity used for cavity ring-down experiments, with a several $\mathrm{kHz}$ linewidth, this is sufficient to shift the laser off resonance without shifting to another cavity mode (172 MHz free spectral range for an $87 \mathrm{~cm}$ cavity). The 30-MHz shift for an index of refraction of 3.5 corresponds to a change in refractive index of $5 \times 10^{-7}$. Using the results from Manning et.al. ${ }^{68}$ the injected power to main laser power ratio is on the order of $10^{-5}$. This is reasonable given optical losses along the pulsed laser path, and uncertainty in the spot size of the pulse beam on the main laser facet.

\subsection{Ringdown Performance (Time-Domain)}

A critical aspect of this approach is that the frequency shift from optical injection of the pulsed laser is sufficient to shift the laser off-resonance, such that an exponential decay is observed. To estimate the extinction ratio (the ratio of the laser power entering the cavity when shifted off-resonance to the power when on-resonance), we estimated a $<1-\mathrm{MHz}$ linewidth of the main laser using the OHD system. Assuming a Lorentzian shape, this results in a minimum extinction ratio of $30 \mathrm{~dB}$ for a $20-\mathrm{MHz}$ shift, and $45 \mathrm{~dB}$ for a $100-\mathrm{MHz}$ shift. Figure 16 shows a typical ring-down triggered after a buildup of energy in the cavity, and then induced by the pulsed laser. The single exponential curve fit is also shown. An additional requirement for CRDS is that the laser-cavity interaction be broken with sufficient rapidity (here 200-500ns) so that a single decay is observed, as demonstrated here by the residuals of the fit (see inset). In subsequent experiments we measured the individual time constants for 17 ring-downs and obtained an average of $12.11 \pm 0.38 \mu \mathrm{s}(1 \sigma)(3.25 \%$ relative standard deviation). Due to the limitations of our 
current data acquisition system, each ring-down was taken approximately 30 seconds apart, suggesting that the variation in time constants are a result of thermal-induced drifting of the cavity length.

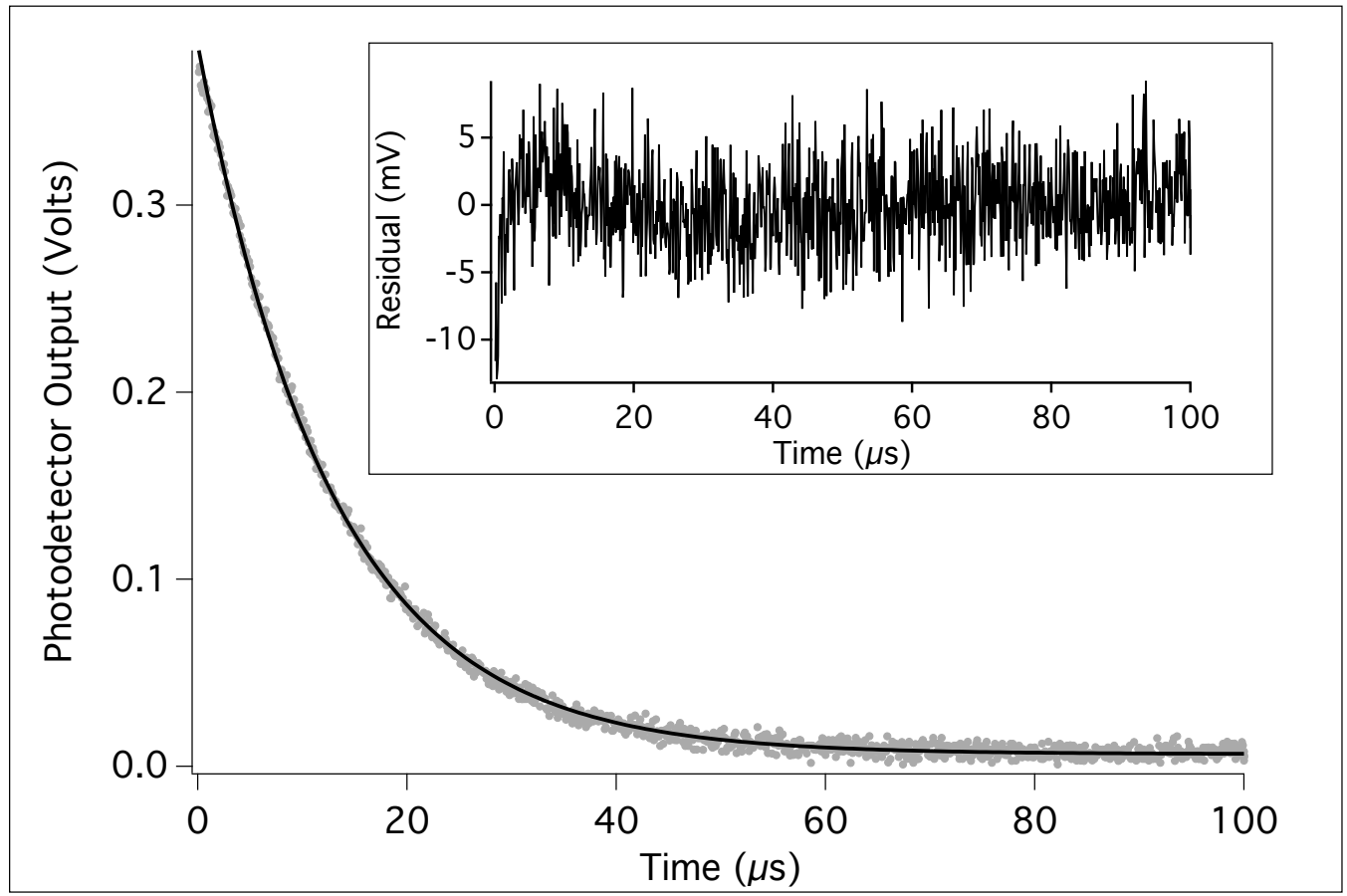

Figure 16 A single ring-down event induced by the pulsed laser frequency shifting the main laser captured on an 8-bit digital oscilloscope (dots). To reduce quantization error, the data was resampled at a rate of 1:5. The exponential fit using a Levenberg-Marquardt algorithm is shown (solid line), as well as the residual to the fit (inset).

Finally, we tested the ability of the technique to repeatedly return the main laser to the resonance frequency of the cavity. We configured the pulsed laser circuit as an astable circuit, producing ring-downs at regular intervals and achieved ring-down decays at a rate of $3.5 \mathrm{kHz}$. Oscilloscope data collected for two consecutive ringdowns is shown in Figure 17. The dashed line indicates the trigger pulse for the pulsed laser. This again demonstrates that the pulsing technique does not adversely affect the main laser frequency, which returns to its initial frequency as soon as the pulsed laser shuts off, 
allowing energy buildup inside the cavity. Further, applied to CRDS, the high data acquisition rates can dramatically improve sampling statistics and precision of measurement (e.g., theoretical improvement of a factor of $\sim 60$ over a $1 \mathrm{~Hz}$ acquisition rate).

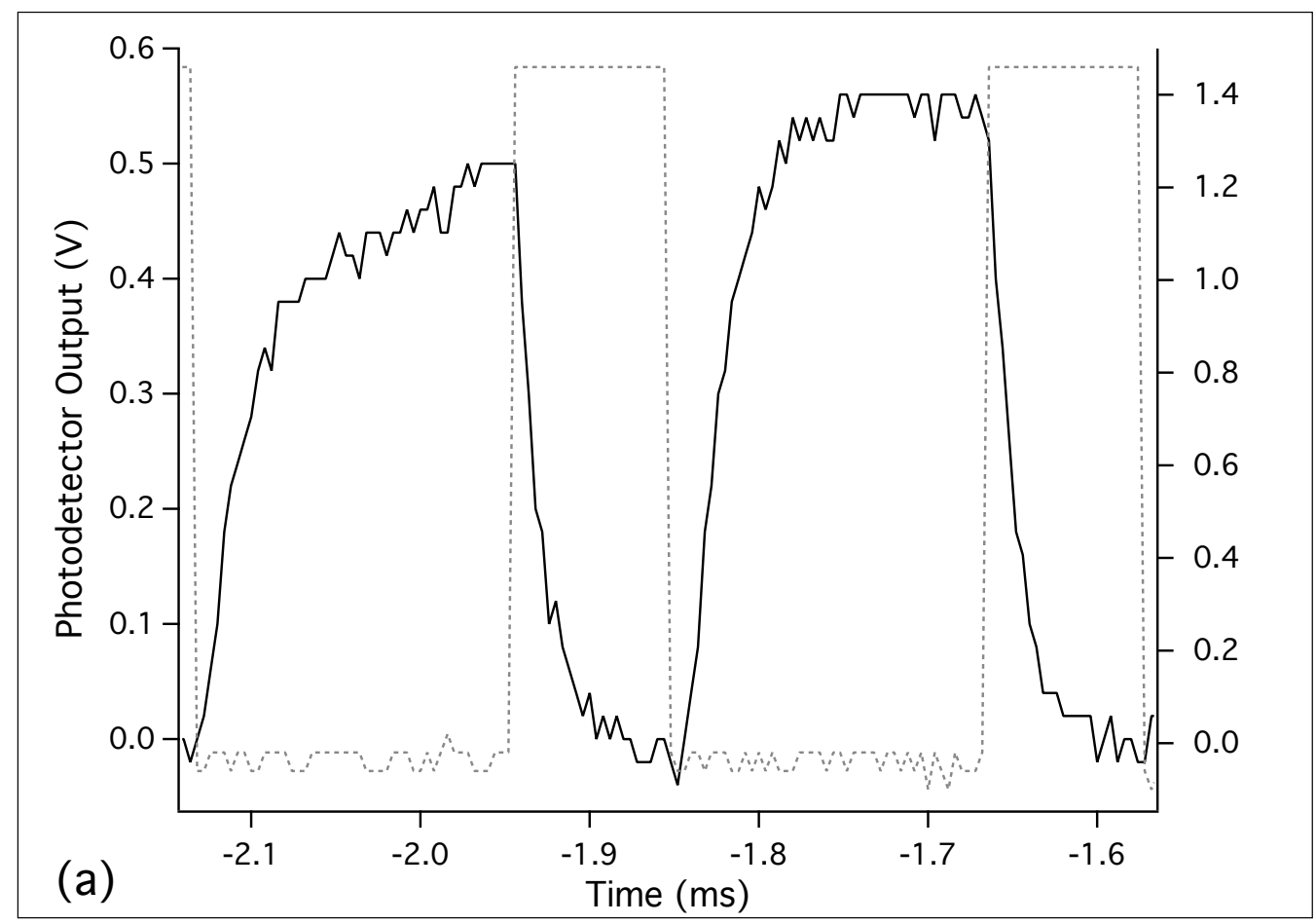

Figure 17 Using the frequency shift for ringdowns. Two consecutive ringdowns are shown, indicating that the main laser locks back to the cavity after the pulse laser is turned off. The dashed line is the digital pulse sent to the laser. 


\section{Chapter 5 FPGA Processing System ${ }^{2}$}

\subsection{Decay Constant Processing}

Cavity ringdown spectroscopy offers improved sensitivity over other absorption techniques $^{45,2}$, but significant data processing is typically required to determine the decay constant. Since sensitivity can be improved by averaging many measurements, the speed at which data can be processed can limit the performance of CRDS systems. Several algorithms exist for decay constant determination, such as the traditional, but computationally intensive, Levenberg-Marquardt non-linear fit. Alternative algorithms such as corrected successive integration (CSI), the linear regression of the sum (LRS), and Discrete Fourier Transform (DFT) algorithms ${ }^{69,70}$ are more efficient, typically orders of magnitude faster than the non-linear fitting.

With fast algorithms, the data-throughput bottleneck becomes getting the signal from the digitizer to the processor. Some applications have avoided the conversion stage altogether by using an analog method of determining the decay constant ${ }^{71}$. Others slow down the transfer by determining the decay constant of an average of many ringdowns, rather than averaging decay constants of many individual ringdowns. However, if the latter has an assumed normal distribution, then the former would have a lognormal distribution, with a mean that depends on both the average $\beta$ and its standard

\footnotetext{
${ }^{2}$ Most of this chapter is taken from a manuscript entitled "The discrete Fourier transform algorithm for determining decay constants-implementation using a field programmable gate array," which has been accepted for publication in Review of Scientific Instruments (RSI) and has been reproduced with permission from RSI.
} 
deviation $^{72,73}$, introducing additional error that can be avoided if individual ringdowns could be processed in real-time. Furthermore, the precision becomes limited by the number of bits of the high-speed data acquisition system, and averaging ringdowns has been shown to enhance low-frequency noise on the ringdown signal, requiring additional processing ${ }^{40}$. Finally, processing individual ringdowns enables removal of outliers that can significantly affect the averaged ringdowns ${ }^{74}$.

Improvements in speed, and therefore sensitivity, can be obtained by "moving" the processing stage closer to the analog-to-digital converter (ADC) stage. High-speed Field-Programmable Gate Arrays (FPGA) provide the possibility of putting a customized, highly efficient processor module immediately after the $\mathrm{ADC}^{59}$ for faster processing of decay constants. Few practitioners of CRDS have applied this approach despite the fact that the ADC and FPGA integrated circuits (IC) can be relatively inexpensive; presumably because of a perception that building the proper support circuitry around them can be time-intensive and require a high level of skill in circuit design. Purchasing commercial high-speed data acquisition modules with (or even without) onboard FPGAs can be costly (several thousand dollars). In this work, we pursued a middle ground between designing our own data acquisition and processing (DAP) system from the ground up and buying a commercially available system. Adapting commercially available hardware designed for high-speed communication applications to make it compatible with the exponentially decaying signals encountered in CRDS provides a cost-effective solution for high-speed DAP to acquire decay constants, which we demonstrate at rates up to $4.4 \mathrm{kHz}$. 
The central components of our system are commercially available evaluation boards from Analog Devices, Inc. (Norwood, MA) designed for the evaluation of their high-speed ADC IC. Additional components used to interface with these boards, as well as the modifications we made to the evaluation boards are discussed in the following sections. To our knowledge, there is only one report of using an FPGA for processing CRDS data ${ }^{59}$, in which an Altera FPGA evaluation board with an ADC daughter card is used to compute the decay constant. In that work they used a modification of the discrete sum/frequency component algorithm, and used a lookup table to determine the time constant from the ratio of two frequency components. Their system was implemented on the FPGA, and they demonstrated its performance at $20 \mathrm{~Hz}$. In addition to a different FPGA and ADC evaluation boards, our approach is different in two fundamental ways: (1) we implement the DFT algorithm described by Everest and Atkinson ${ }^{70}$ using an FFT core to determine the decay constant without use of a lookup table and (2) we have modified the input path to the ADC to pass low frequencies, avoiding the signal distortion caused by the transformer seen in the ringdowns of Spence et. al., which may have contributed to that system's lack of agreement with the spectrophotometer data ${ }^{59}$.

\subsection{The FFT algorithm}

The DFT algorithm for determining CRDS decay constants has been described previously $^{20,70}$. The basis of the algorithm comes from the Fourier Transform of an exponential decay function with decay constant $\beta$, given by (for $t>0$ ):

$$
f(t)=e^{-\beta t}
$$

The complex Fourier transform is found to $\mathrm{be}^{75}$ : 


$$
\begin{aligned}
F(\omega) & =\frac{1}{\beta+i \omega} \\
& =\frac{\beta}{\beta^{2}+\omega^{2}}-i \frac{\omega}{\beta^{2}+\omega^{2}}
\end{aligned}
$$

Here, $\omega$ is the angular frequency $(\mathrm{rad} / \mathrm{s})$, and the transform has been broken into its real and imaginary parts. Taking the ratio of the real and imaginary parts gives a simple expression for the decay constant:

$$
\beta=-\omega \frac{\operatorname{Re}(F(\omega))}{\operatorname{Im}(F(\omega))}
$$

Thus the decay constant for an exponential decay can be found using any frequency component of the Fourier transform. The first non-zero frequency component is typically chosen to maximize the signal-to-noise ratio (SNR), since the real and imaginary parts have their maximum at $\omega=0$, and $\omega=\beta / 2 \pi$, respectively, and drop to $10 \%$ of their peak value in less than $\omega=5 \beta / 2 \pi$ and $\omega=20 \beta / 2 \pi$, respectively. This means that for a $10 \mu$ s decay time $\left(\beta=10^{5} \mathrm{~s}^{-1}\right)$, the SNR for each part is an order of magnitude lower at $80 \mathrm{kHz}$, and $320 \mathrm{kHz}$, respectively. Note that low frequency distortion of the signal can have a significant impact on the time constant (and therefore the analyte concentration) determined by this method.

In practice, a Discrete Fourier Transform (or an FFT) is applied to digitized data, which results in a small error when using the simple relationship in Equation (13), but can be corrected by using the ratio in a slightly more complicated equation ${ }^{70}$ :

$$
\beta=\frac{1}{\Delta t} \ln \left[\frac{\operatorname{Re}(F(k))}{\operatorname{Im}(F(k))} \sin \left(\frac{2 \pi}{N} k\right)+\cos \left(\frac{2 \pi}{N} k\right)\right]
$$


where $k$ is the frequency index, $N$ is the total number of time series samples, $\Delta t$ is the sampling interval (10 ns for a $100 \mathrm{MHz} \mathrm{ADC}$ clock), and the frequency $f($ in $\mathrm{Hz}$ ) for any $k$ is given by $f=k /(N \Delta t)$. In this work, in order to conserve FPGA resources and increase the processing speed, we only computed the ratio of the real and imaginary parts on the FPGA. This ratio can then be used in either Equation (13) or (14) to calculate the time constant on the computer. For this work we used Equation (13), resulting in less than a $0.1 \%$ difference from Equation (14), when using the first frequency component $(k=1)$.

\subsection{Hardware configuration}

A block diagram of the data acquisition system is shown in Figure 18. The signal from the photodetector that measures the decay of the light from the cavity enters a circuit that level-shifts and attenuates the signal to levels appropriate for the ADC. The signal is then passed to an AD9255 Evaluation Board (AD9255-105EBZ, Analog Devices, Inc.) containing a 14-bit pipelined, switched-capacitor ADC that can run at sampling rates up to 125 MSPS. The AD9255 evaluation board is designed with a highspeed interface to FPGA-based buffered memory boards (Analog Devices, Inc.). We chose the HSC-EVALCZ, using a Virtex-4 FPGA (Xilinx, Inc. San Jose, CA) that can read up to 16 bits from the upstream evaluation board (of which our ADC supplies only the upper 14 bits). The ADC continuously supplies data to the FPGA (i.e., it is not triggered to start the ADC process) with a synchronous clock to signal the FPGA to read in the data. The FPGA board is triggered by a digital signal from the same threshold circuit that induces the ringdown event; in our system, it triggers a pulsed diode laser to

shift the main laser off-resonance from the cavity ${ }^{46}$. After acquiring data for a fixed time 
period (corresponding to $\sim 51 / \mathrm{e}$ times for the ringdown decay) the FPGA performs a discrete FFT on the ringdown data and divides the first real and imaginary components (i.e., Eq. 13). The FPGA then sends the 16 most significant bits of the result through a digital level converter to an Arduino Due microcontroller (Arduino, SA., Turin, Italy) for further processing. The Arduino Due stores the values in memory and then transmits them via USB to the computer.

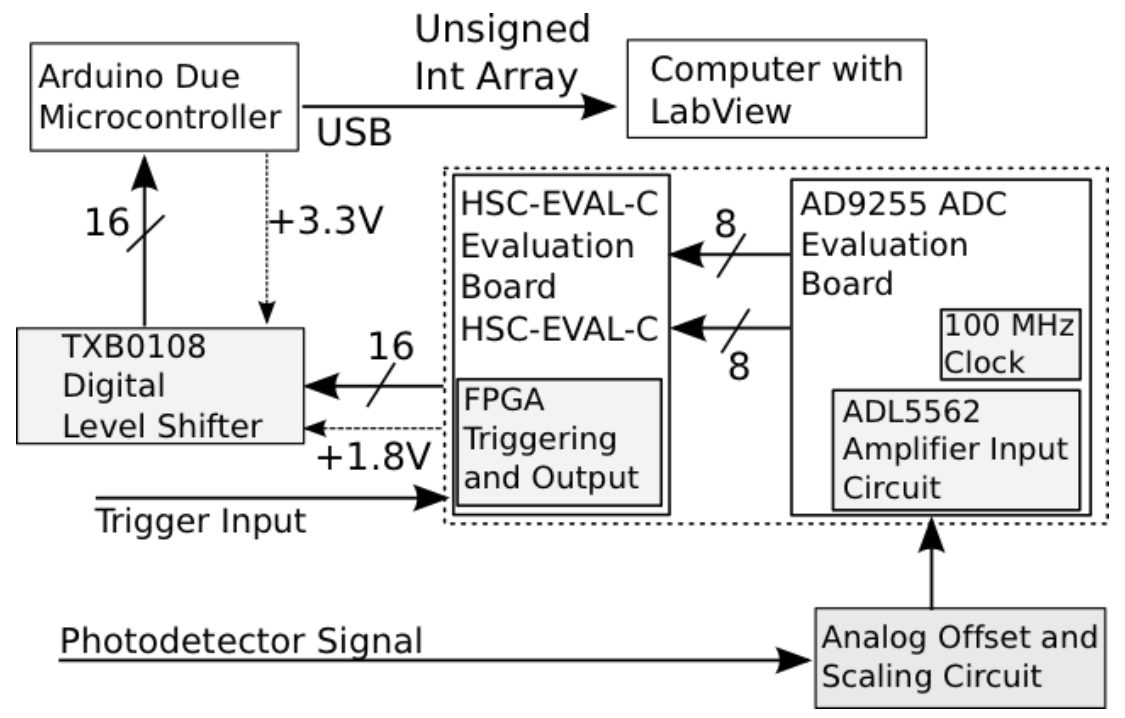

Figure 18 Block Diagram of DAQ system, whose main components are the AD9255 ADC evaluation board and the co-requisite HSC-EVALC board containing the FPGA. The blocks in gray are the additional circuits or evaluation board modifications discussed in the text.

The required modifications to the ADC and FPGA evaluation boards are discussed in Sections 5.4 and 5.5, respectively, with circuit details deferred to Section 5.9. The description of the digital level shifter is included in Section 5.4. The analog scaling and offset circuit is described in Section 5.6 (with circuit details also in Section $5.9)$. 


\subsection{AD9255 evaluation board modifications}

Most high-speed ADCs require differential inputs (paired signals with equal magnitude and opposite sign). Typically photo-detectors used for CRDS are single-ended outputs (i.e., referenced to ground). Therefore, in order to make best use of the ADC, a differential signal must be generated from this single-ended signal. The majority of highspeed ADC/FPGA applications are related to communications or signal processing of sinusoidal waves at $\mathrm{MHz}$ frequencies and above. We found that as a consequence the evaluation boards are designed for AC-coupled, high frequency input signals where the key features of a ringdown signal are a relatively low frequency decay followed by a DC level between excitations. The evaluation board's standard input path includes conversion of a single-ended signal to a differential signal using a radio frequency (RF) transformer that is designed to work with alternating signals that, as stated in the AD9255 data sheet, "can saturate at frequencies below a few megahertz."76 The effect of this is attenuation of low frequencies, or high-pass filtering, and our initial attempts to use the standard input path resulted in ringdown signal distortion similar to that reported by Spence et.al. ${ }^{59}$ Fortunately, the evaluation board contained a secondary input path to the AD9255 with an operational amplifier (ADL5562, Analog Devices, Inc.) capable of converting DCcoupled, low frequency, single-ended signals to the requisite differential signals. Unfortunately, the majority of the alternate input path is unpopulated, and the components that were installed assumed an AC-coupled signal. The modifications needed to make this path functional for ringdown signals are detailed in the 5.9.1, and follow the recommendations given in the data sheets for the AD9255 ${ }^{76}$ and the ADL5562 ${ }^{77}$. 


\subsection{FPGA evaluation board configuration}

In our design the AD9255 continuously digitizes the input signal, regardless of whether a ringdown has been initiated, and it is the FPGA that is triggered to start storing the digitized data and begin the FFT processing. A threshold-triggering circuit enables both the laser unlocking ${ }^{46}$ (to initiate the ringdown) and the FPGA data processing. For the FPGA, a solid-state switch and a 1.5-V input created from a simple voltage divider was used since 5-V TTL levels used for the other parts of our system are incompatible with the 1.8-V logic on the FPGA evaluation board. The FPGA evaluation board has an 18-pin 0.1"x0.1" header designated as debug pins which allows access to FPGA inputs. In this case the trigger signal is connected to debug pin 14 (maps to FPGA pin AB7) which is registered as a digital input buffer in the FPGA logic.

The FPGA evaluation board outputs the 16 most significant bits of the ratio of the real and imaginary parts of the first component of the FFT as described in Section 5.7.1. Although the FPGA evaluation board has an onboard USB transceiver for communication with a host computer, a significant number of gates are needed to communicate with the transceiver. Because we opted for a number of quality control/debugging capability in this version of the system those gates were not available, we chose to use an Arduino Due microcontroller as an interface between the FPGA and the computer. The bottom side of the FPGA board has soldering pads for 3 90-pin $0.05 " x 0.05 "$ dual-line headers, designed to connect to a Digital Signal Processing daughterboard. Several of these pads are routed to FPGA pins so we used these to transmit the 16-bit representation of the calculated ratio off the FPGA evaluation board. Additional pins are available on the evaluation board, so it is possible to expand the 
output by another 8 bits to increase the precision of the ratio, if this approach is adopted. Alternatively, some of the debugging capabilities could be sacrificed to allow for direct connection to a computer via the USB.

The FPGA board uses $1.8 \mathrm{~V}$ digital logic while the Arduino Due uses 3.3V digital logic, so we used two 8-Channel Bi-Directional Digital Level Converters (Adafruit Industries, New York, NY) to provide the Arduino Due with the proper digital levels. These are small daughter boards designed to be mounted in a larger module, so we set them up external to the FPGA board, as shown in Figure 18. The Arduino and the FPGA evaluation board each supply power for their respective side of the level converter, while the ground is tied to the Arduino. Details of the mapping of the 16 bits of the FPGA output through to the Arduino digital input buffers are given in Table 1 of Section 5.9.3.

\subsection{Analog offset and scaling circuit}

Common mode voltage incompatibility required additional circuitry to avoid distortion of the output signal. Specifically, for "best distortion performance" in DC applications, the input common mode voltage - the average of the positive (noninverting) and negative (inverting) inputs-should be in the range $1.0 \mathrm{~V}-2.3 \mathrm{~V}^{77}$. The ringdown is a DC-coupled, ground-referenced signal; and, for our system, the maximum voltages were in the range $0.2 \mathrm{~V}-1.0 \mathrm{~V}$, making the common mode voltage significantly below the recommended range. In addition, as shown in Figure 21 of Section 5.9.1, the output common mode of the ADL5562 amplifier is set to the AD9255 input common mode voltage of $0.9 \mathrm{~V}$, making it also below the recommended range of $1.25 \mathrm{~V}-1.85 \mathrm{~V}^{77}$. Due to these mismatches in input and output common mode voltages, the ringdown 
signal measured by the ADC exhibited distortion, as seen in the ringdown in Figure 19(a). The distortion, most likely attributed to phase reversal ${ }^{78}$ of the larger voltages at the beginning of the ringdown, makes a significant portion of the decay signal unusable. To more properly condition the signal, we first used a simple potentiometer-based voltage divider to reduce the amplitude of our ringdowns to be within the undistorted range (approximately $70 \mathrm{mV}$ or less). We then increased the undistorted range using a subtraction circuit ${ }^{79}$ to add a negative offset to the signal, matching it to the input signal range of the ADL5562 amplifier. The offset and scaling circuit is described in more detail in Section 5.9.1, and can be adjusted to maximize the signal levels and minimize the distortion. A ringdown taken using the circuit is shown in Figure 19(b), and comparison with Figure 19(a) shows the improvement in amplification and digitization fidelity. The resulting ringdown is undistorted, improving the fit (the residual is shown in the inset of each figure), increasing the signal-to-noise ratio (since points with larger voltage are available), and increasing the total number of data points used in the fit.
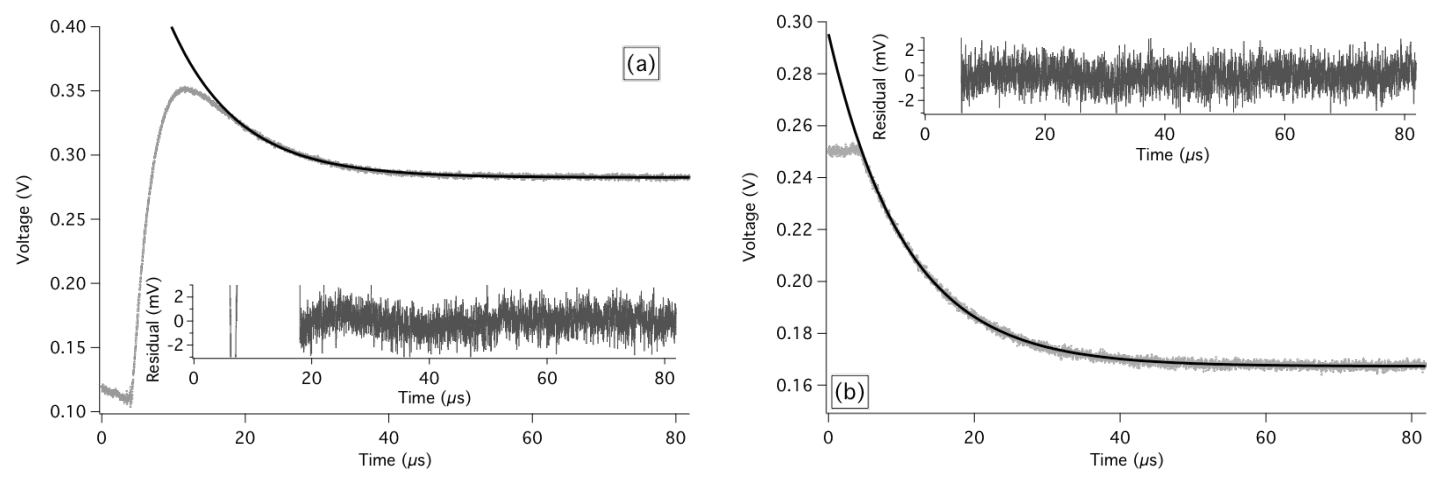

Figure 19 Comparison of FPGA captured ringdown with and without the ADC front end circuit. (a) The gray line shows the photodetector output (triggered at $440 \mathrm{mV}$ ) passed directly to ADC evaluation board, which exhibits distortion due to the low common mode voltage set by the AD9255 amplifier. (b) This shows a signal at the same trigger level passed through the scaling and offset circuit, which eliminates the phase reversal distortion. The front end expands the range of valid data in both voltage and in the number of points available for determining the decay constant. Exponential curve fits (black lines) are shown in both (a) and (b) to highlight the distortion, and the residual to the fit is shown in the inset. 


\subsection{Software configuration}

This section discusses the software components used for processing and control of both the FPGA and the Arduino Due, and the code for them are provided in Appendix C and $\mathrm{D}$, respectively.

\subsubsection{FPGA coding description}

In order to program the FPGA we used the Xilinx ISE® Design Suite, an integrated development suite for coding, pin assignments, debugging, and loading the FPGA with design. Its use enabled access to the Xilinx LogiCORE ${ }^{\mathrm{TM}}$ IP Fast Fourier Transform core ${ }^{80}$ and the LogiCORE ${ }^{\mathrm{TM}}$ IP Divider Generator core $^{81}$. Since the evaluation board had all of the FPGA input/output pins routed, we were constrained by its initial design as to which pins we could use for processing and output. The FPGA code timing was set to the $100 \mathrm{MHz}$ synchronous clock onboard the ADC evaluation board. After the external trigger input buffer is set high (see Section 5.5), the code includes a delay from the rising edge of the trigger before starting the data processing. This delay is configured to avoid any transients in the laser switching that may be present in the ringdown signal. In our configuration, an acceptable delay was $1.6 \mu$ s. The ADC samples are then loaded directly into the FFT core, and processing begins as soon as the last sample of the ringdown is taken. The FFT core was configured for an 8192-point 16-bit integer transform. At $100 \mathrm{MHz}$, the 8192 samples captures $81.92 \mu$ s of ringdown signal—a collection time long enough to ensure optimal performance of the DFT algorithm (4-5 time constants) $)^{70}$. To provide maximum transform fidelity the algorithm was configured as unscaled, meaning that the number of bits at the output expands to 28 bits (due to the 
multiplications involved) and the least significant bits are not dropped as the information progresses through the algorithm.

Upon completion of the FFT, a DONE flag and a DATA VALID flag are set high, and on each clock cycle the real and imaginary parts of each frequency component in the transform are output in order. Once the desired component is output ( $k=1$ in this work), the values are latched, and the integer division core is enabled. The division core was configured as a 28 -bit by 28 -bit division, with the result having a 28 -bit integer part and a 14-bit fractional part. The division core required 32 clock cycles to complete, after which the integer and fractional part are latched at FPGA output pins. Given that typical decay constants are on the order of $10^{5}$, we find that the ratio generated should be on the order of 1 (see Equation (13)), meaning that most of the 28 bits of the integer part will be zero. We chose to keep 3 bits of the integer part, and 13 bits of the fractional part, providing a maximum time constant of $6.1 \times 10^{5} \mathrm{~s}^{-1}$ with a resolution of approximately $9 \mathrm{~s}^{-1}(0.01 \%$ for $\beta=10^{5} \mathrm{~s}^{-1}$ ). For our CRDS instrument this is equivalent to a maximum absorption of 0.016 (a maximum absorption coefficient of $1.9 \times 10^{-4} \mathrm{~cm}^{-1}$ ), and an absorption sensitivity of $2.6 \times 10^{-8}$ (or a minimum absorption coefficient of $3 \times 10^{-10} \mathrm{~cm}^{-1}$ ) which, based on the results in Chapter 6, impacts neither the peak absorption nor the sensitivity of the rest of the system. Once the division core completes, the FFT core is reset, and the FPGA is ready to capture the next ringdown. The ratio result is latched at the output until it is reset by the next processed ringdown, so that the result can be read at a slower rate. The total processing time on the FPGA was measured to be $145 \mu \mathrm{s}$. Combining that with the ringdown time of $81.92 \mu \mathrm{s}$, the FPGA should be able to process ringdowns at a maximum rate of $4.4 \mathrm{kHz}$. 


\subsubsection{Arduino Due storage and post-processing}

The Arduino Due is an open-source microcontroller with a 32-bit processor, an 84 MHz clock, 54 digital input/output pins, and a USB interface for programming and communication with a computer. We used 16 of the digital input buffers, mapped to the output bits of the FPGA division calculation (See Table 1 of the Section 5.9.3) and conditioned as described in Section 5.7.1. The Arduino reads digital input as a 16-bit unsigned integer and stores it in memory in approximately $22 \mu \mathrm{s}$. This is less than the time to load and process the next ringdown and thus does not form a bottleneck in the processing. The memory limitations on the Arduino restrict the number of stored 16-bit values to approximately 150 . The Arduino storage and transmit functions are controlled by commands from the computer via USB. One command initiates the storage of ratio values from the FPGA, and a second command stops the storage, and transmits all stored values to the computer (as a series of strings). For this work, we were interested in recording each individual decay constant, but it would be straightforward to have the Arduino Due compute the average, and only pass that to the computer. The 16-bit unsigned integer value, $\beta_{\text {raw }}$, becomes the ratio required in Equations (13) and (14) by dividing by $2^{13}$ to move the decimal place, and then converting to the decay constant $\beta$ (in $\mathrm{s}^{-1}$ ) by multiplying by $\omega_{1}=2 \pi /(N \Delta t)$. In our system $\beta=2 \pi \beta_{\text {raw }} /\left(81.92 \times 10^{-6} \times 2^{13}\right)$, which simplifies to $\beta=9.3626 \beta_{\text {raw }}$.

\subsection{Performance of FPGA Processing}

The FPGA processing was incorporated into a CRDS system described in Chapter 4 and Bostrom et.al. ${ }^{46}$. The cavity was $85 \mathrm{~cm}$ long with a finesse in excess of 10,000 . A 
computer running LabView stepped the main laser current, shifting the laser frequency by $1.06 \mathrm{GHz} / \mathrm{mA}$ to the predicted cavity mode and then used a dithering algorithm to find the cavity modes. The LabView code is described in Section 6.1. An absorption spectrum of humid air, at a cell pressure of 500 torr and temperature of $22^{\circ} \mathrm{C}$, was obtained by collecting up to 150 samples per frequency point/collection time as shown in Figure 20. The two peaks shown are absorption peaks of $\mathrm{H}_{2} \mathrm{O}$ in the near infrared, with the largest centered near $7640.8 \mathrm{~cm}^{-1} .{ }^{1}$ A Voigt line-shape function was fitted to the two Figure 20 peaks, and the residual is shown. The median relative standard deviation for the fit of the data points was $1.9 \%$ (mean RSD was $3.1 \%$ ). In our system the instability of the lasercavity locking resulted in different data collection rates at different cavity modes/frequency positions, but the median number of samples per mode was 150 (mean $=115$ ), meaning that the overall system collected decay constants at a rate of at least 1.5 kHz. This is within a factor of 3 of the maximum capability of the FPGA, although the number of samples collected at each collection time/frequency point was limited to 150 by the Arduino memory. 


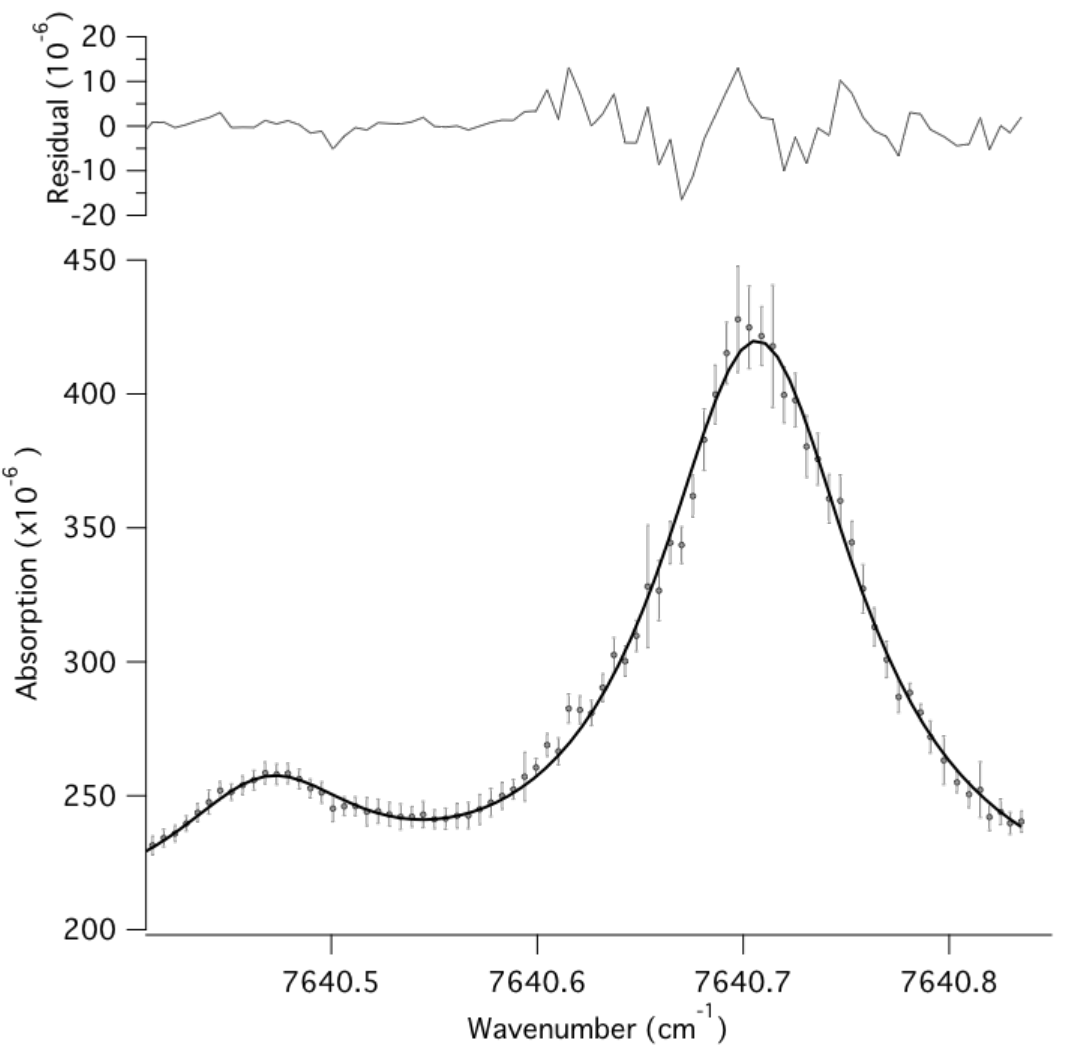

Figure 20 Near infrared spectrum of $\mathrm{H}_{2} \mathrm{O}$ obtained using decay constants calculated on the FPGA. The average of the samples taken at each wavenumber are shown as dots, with error bars indicating \pm one standard deviation for that point. Voigt lineshapes were applied to each of the two peaks, and the solid line indicates the fit, while the residuals are shown above the spectrum.

In total the method presents an optimized cost-effective data acquisition system for CRDS that is capable of determining time constants at a maximum rate of $4.4 \mathrm{kHz}$ by modifying a commercial ADC-FPGA evaluation board, and programming it to apply the DFT algorithm for determining decay constants. We have provided the details of the modifications with generally available hardware, so that the system could be incorporated into existing CRDS instruments to enable faster data collection and processing.

In this initial version, significant FPGA resources were used for debugging and evaluating/documenting performance, and so only one FFT component was used for the decay constant. Future implementations would need fewer resources for debugging, 
which could then be used for improvements to the system. Straightforward modification of the FPGA code to use the average of ratios for the first 5 components would improve the precision, which is designated as the DFT-5 algorithm in Everest and Atkinson ${ }^{70}$. Averaging, pipelining of the FFT core to increase the processing rate, and communication via the on-board USB transceiver could also be implemented with additional FPGA resources.

\subsection{Technical Details: Hardware Modification}

\subsubsection{ADC evaluation board modifications}

A circuit diagram highlighting the ADC board modifications is shown in Figure 21. The rectangles indicate resistors and capacitors that were already included on the board. All others were added based on recommended values for frequencies between 0 and $100 \mathrm{MHz}^{76}$ and assume a source impedance of $50 \Omega^{77}$. The names for specific resistors, capacitors, inductors, and jumpers in the following discussion follow the identifications provided in the User's Guide for the ADC evaluation board ${ }^{82}$. The resistors and capacitors installed are surface mount devices (SMD), size 0402 (1 mm x $0.5 \mathrm{~mm}$ ), and the soldering and rework was done using a hot air rework station. The following pre-installed capacitors were removed: $\mathrm{C} 2, \mathrm{C} 4, \mathrm{C} 12, \mathrm{C} 14, \mathrm{C} 71, \mathrm{C} 96$, and $0 \Omega$ resistors were installed at the following capacitor locations: C12, C14, C49, C50, C51. Capacitor C15 was replaced with an 18 pF capacitor. Resistors R3, R8, R22, and R23 were replaced with $15 \Omega$ resistors. R15 and R16 were removed, and R13 was replaced with a $26 \Omega$ resistor. R54 (0 $\Omega), \mathrm{R} 20$ and R21 (1k $\Omega)$ were installed, and locations R18, 
R19, L8, and L9 were shorted. The $33 \Omega$ resistors in Figure 21 were installed at locations L5 and L6. The board is factory-configured for an external clock input, but comes with circuitry for an onboard clock. We installed a compatible 100-MHz clock (OCA3H-C3A100.000M, Ascend Frequency Devices, Lake Oswego, OR), replaced C70 with a $0 \Omega$ resistor, and removed R25, following the instructions in the user's guide ${ }^{82}$.

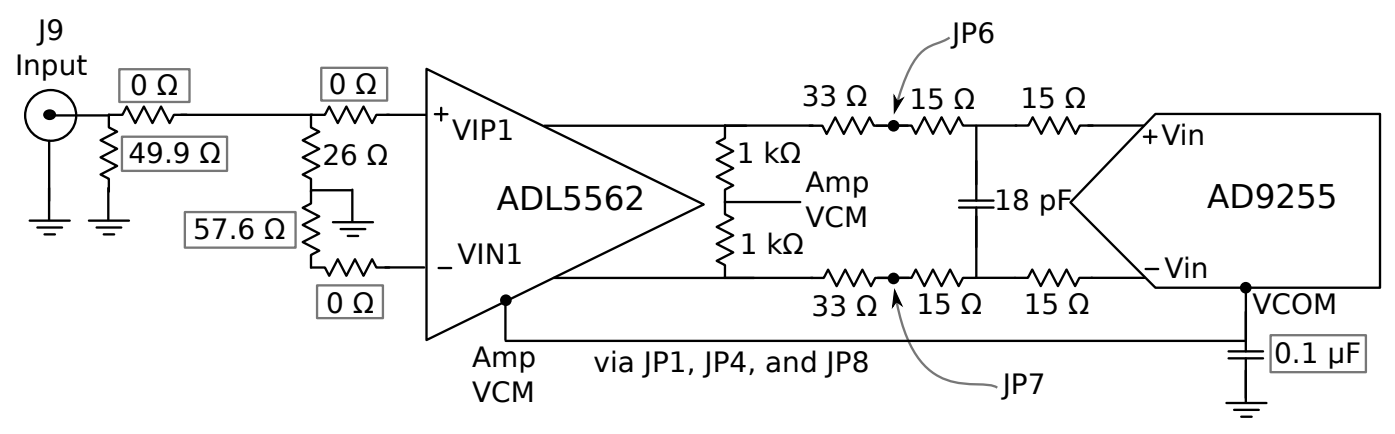

Figure 21 Circuit Diagram of the amplifier input path to the ADC. The rectangles around the labels indicate components that were already on the circuit board, and were not modified. The other resistors and the 18 $\mathrm{pF}$ capacitors need to be installed on the board.

On the evaluation board, the solder jumpers (JP1, JP4, JP8) are shorted to connect the common mode voltage output of the AD9255 to the common mode input of the ADL5562, and the midpoint of its output. JP6 and JP7 were shorted to connect the ADL5562 to the AD9255 input path. Additional header pin jumper settings on the ADC board are as follows: P19 left open enables the ADL5562; P5 (SENSE) 1 $\rightarrow$ 2, sets ADC voltage reference to $0.5 \mathrm{~V}$; installing P6 enables the onboard clock; P4 $2 \rightarrow 3$ enables duty cycle stabilizer (DCS); P4 5 $\rightarrow 6$ enables two's complement; P14 open enables Low Voltage CMOS digital output signals to the HSC-EVALC board; P13 open disables dithering. 


\subsubsection{Analog offset and scaling circuit}

The circuit used to scale and provide an offset to the photodetector output (to eliminate the signal distortion due to the common mode voltage phase reversal) is shown in Figure 22. We used a simple subtraction circuit $^{79}$, based on a LF356N operational amplifier. A potentiometer between $+5 \mathrm{~V}$ and ground creates a voltage divider allowing us to vary the subtracted DC voltage. The scaling is accomplished with another voltage divider, which outputs a fraction of the offset photodetector signal to the ADC evaluation board. The potentiometers are adjusted to ensure the ADC input is in the proper range to maximize fidelity of conversion while preventing signal distortion.

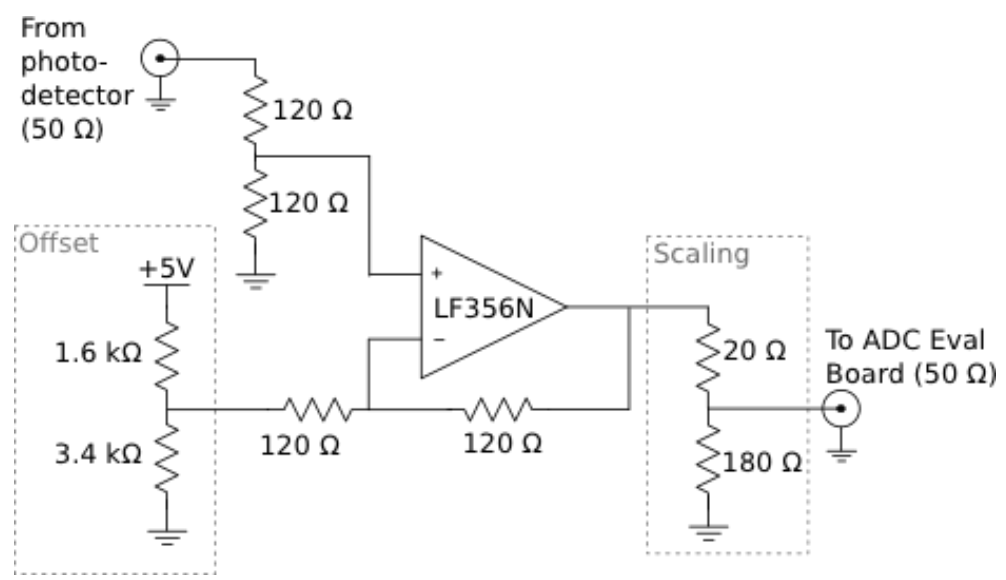

Figure 22 Diagram of the analog offset and scaling diagram to the AD9255 Evaluation Board. An adjustable offset is subtracted from the output of the photodetector, and the result is scaled using a voltage divider so that the ringdown is within the voltage limits of the ADC. The resistors in the offset and scaling dashed rectangles are made using the center tap of a $5 \mathrm{k} \Omega$ and $200 \Omega$ variable resistor, respectively.

\subsubsection{FPGA board modifications and pin mapping}

To transmit the result computed by the FPGA, we used one of three solder pads designed for connecting a Digital Signal Processing (DSP) daughterboard through 90-pin $0.05 " x 0.05 "$ dual-line headers. We soldered a 28-position header (FTSH-114-01-L-DV, 
Samtec, Inc., New Albany, IN) to pins 3 through 32 of the P1 pad ${ }^{82}$. A cable assembly (SFSD-15-28-H-10.00-SR, Samtec, Inc.) with a corresponding 30-position socket on one end and bare wires on the other was then used to connect the FPGA output pins to the 0.1"x0.1" headers on the Digital Level Shifter (See Figure 18). Table 1 maps the data lines corresponding to the bits of the FPGA ratio calculation to the pins of the FPGA board and the Arduino microcontroller. These mappings correspond to our code written for the FPGA and Arduino.

TABLE 1 Arduino Due to FPGA Translation. The names in Columns 2-5 correspond to those given in the Evaluation board User's Guide ${ }^{82}$. The Arduino Due GPIO pins correspond to the labeling on its circuit board.

\begin{tabular}{llllll}
\hline \hline $\begin{array}{l}\text { Re/Im } \\
\text { Output Bit }\end{array}$ & Eval & Board FPGA Pin & \multicolumn{2}{l}{ Eval Board Level } & Arduino \\
ScheadicName & & Header & $\begin{array}{l}\text { Pin } \\
\text { Shifter } \\
\text { Pin }\end{array}$ & $\begin{array}{l}\text { Due } \\
\text { GPIO Pin }\end{array}$ \\
\hline \hline 0 (LSB) & DSP_A0 & AF15 & 5 & A-A0 & 2 \\
1 & DSP_A2 & AF14 & 7 & A-A1 & 3 \\
2 & DSP_A4 & Y8 & 9 & A-A2 & 4 \\
3 & DSP_A6 & AA13 & 11 & A-A3 & 5 \\
4 & DSP_A8 & AA12 & 13 & A-A4 & 6 \\
5 & DSP_A10 & AB14 & 15 & A-A5 & 7 \\
6 & DSP_A12 & AA10 & 17 & A-A6 & 8 \\
7 & DSP_A14 & AB12 & 19 & A-A7 & 9 \\
8 & DSP_A1 & AD15 & 6 & B-A0 & 10 \\
9 & DSP_A3 & AE15 & 8 & B-A1 & 11 \\
10 & DSP_A5 & Y7 & 10 & B-A2 & 12 \\
11 & DSP_A7 & AA9 & 12 & B-A3 & 13 \\
12 & DSP_A9 & AA14 & 14 & B-A4 & 22 \\
13 & DSP_A11 & AC14 & 16 & B-A5 & 24 \\
14 & DSP_A13 & AD14 & 18 & B-A6 & 26 \\
15 & DSP_A15 & AC13 & 20 & B-A7 & 28 \\
\hline
\end{tabular}




\section{Chapter 6 Results of System}

The motivation of this work was the development of a cavity ringdown system with high precision while using novel techniques to significantly reduce the cost of the system. Such a system would be well suited to in-situ gas measurement applications, where air sampling becomes impractical. The ability to quickly acquire spectra with high precision makes it more practical in experiments where the gas concentration changes quickly, such as flux measurements, and kinetic isotope effect reactions.

There are a number of advantages inherent in the approach used in this work. Since uncertainty in the mean decreases with the number of measurements, precision (for the same time interval) is improved with faster collection. We have demonstrated an acquisition rate of $1.5 \mathrm{kHz}$, a dramatic improvement of most spectroscopic techniques employed in atmospheric science and among the fastest. Another advantage of our design is that it determines the decay constant of in individual ringdown in near real-time, allowing a larger number of decay constants to be averaged at the same mode. This is superior to averaging ringdowns in that the signal-to-noise ratio is not limited by the quantization of the acquisition system. Our system's ability to acquire ringdowns at $\mathrm{kHz}$ rates is also an advantage over swept-cavity-mode methods since drifting of cavity mode frequencies due to temperature, pressure, and mechanical oscillations limits the number of averages they can use to improve their precision. Finally, high-speed acquisition improves signal to noise ratio and so sensitivity is enhanced in this approach. 
Having assembled and tested the components of the system described in Chapter 5 , we were then ready to evaluate the performance of the entire system in terms of its ability to measure absorption coefficients and gas concentrations. In this chapter, we look at the overall performance of the CRDS system composed of the component modules described in Chapter 2. Before we discuss the results of the system, we first describe the operation and control of the overall system. Then, the acquisition rate, accuracy, precision, limit of detection (sensitivity), repeatability, and linearity are presented in the following sections.

\subsection{System operation and control}

Once the system components were assembled, in order to obtain an absorption spectrum, the main laser current control and communication with the FPGA acquisition system required software integration. A desktop computer using a custom LabView program controlled the overall system. The user interface, displaying actual data, is shown in Appendix F. This section provides a brief description of this code. The basic process flow was:

(1) The laser was set to the desired initial current using the DAC1220, and the "Initiate Collection Signal" was sent to the Arduino Due connected to the FPGA (See Section 5.7)

(2) After a specified delay $(\sim 10 \mathrm{~ms})$, the code checked for any recorded ringdown time constants processed by the FPGA by sending the "Stop and Transmit Signal" to the Arduino Due. 
(3)

(a) If the number of time constants was above a specified threshold, then it waited a specified longer period $(\sim 100 \mathrm{~ms})$ to fill up the Arduino memory. If the number was not sufficient (indicating the laser is likely not staying on the cavity resonance mode), the program moves to step (3b).

(b) If there are insufficient recorded time constants at the laser current, the program began to dither the applied voltage until it obtains sufficient ringdowns to go to step (3a). The dither was a sinusoidal oscillation around the current voltage setting, with exponentially increasing amplitude. The number of oscillations, number of points, and maximum amplitude of the dither was controlled by the user. If the specified number of points had been reached without sufficient time constants collected, the dither timed out, and went to Step (5).

(4) The program read the time constants, and calculated the mean, standard deviation, and the number of ringdowns at each point, and plotted each of them on a graph in real-time. The array of raw time constants at that particular frequency were saved in a text file for additional post-processing.

(5) After collecting the ringdowns, or if the dither timed out, the program stepped the voltage to the next expected cavity mode. Since the cavity modes were equally spaced by approximately $172 \mathrm{MHz}$, The shift in applied voltage, also controlled by the user, was set to $\pm 0.031 \mathrm{~V}$. 
(6) After setting the new voltage, the program returned to step (2), to search for a ringdown at the new voltage. This loop was repeated until the stop voltage was reached.

(7) Once the stop voltage was reached, the plotted data was saved to a timestamped file for post-processing.

The entire program could be run repeatedly unattended, once it had been configured. The main program allowed the user to specify the start and stop voltage of the laser's applied voltage to set the frequency range for the desired spectrum. The user also set the steps per volt (which set the precision) for the applied voltage. These settings controlled the DAC1220 DAC discussed in Section 3.3, and a LabView program module translated the users value to the required SPI command structure.

The trigger threshold level was also controlled by the user, since the output power of the cavity is proportional to the laser power. Laser power increases linearly with current, so we allowed the trigger level to vary linearly with the applied voltage, with the slope and intercept set by the user. The settings were adjusted to balance the ringdown acquisition rate and the laser-cavity locking. With too low of a trigger, and the output level was met before the laser was on resonance with the cavity, and with too high of a setting, the threshold was not always met when the laser was locked to the cavity.

\subsection{Acquisition rate}

In our system, the combination of the cavity locking and the pulsed-laser unlocking, brought the laser back into lock with the cavity immediately after the pulse. The rate at which ringdowns occurred was limited by the time constant of the decay and 
the duration of the collection time-in the current system this limit was $3.5 \mathrm{kHz}$. Our FFT-FPGA acquisition rate was limited by the processing time of the FPGA, which had a maximum rate of $4.4 \mathrm{kHz}$. However, the limiting factor in our prototype design was the memory storage in the Arduino Due, and in the current configuration set our operational limit at $1.5 \mathrm{kHz}$. The acquisition of an entire spectra using the LabView code described in Section 6.1 took approximately 1-2 minutes, a time that depended mainly on the speed that successive cavity modes were located.

\subsection{Comparison with theoretical absorption spectra}

In order to determine the accuracy of our system, we converted our decay constant to an absorption coefficient. The conversion from the time constant, $\beta$, to the fractional absorption is $A=\beta L / c$, where $L$ is the cavity length, and $c$ is the speed of light. From the fit parameters we obtain a value that is proportional to the concentration. Following the HITRAN documentation ${ }^{83}$ :

$$
\begin{aligned}
& A_{e}(v)=A_{e, p k} \frac{\gamma^{2}}{\gamma^{2}+\left(v-v_{0}\right)^{2}}=A_{e, p k} \pi \gamma \cdot f\left(v, v_{0}\right) \\
& f\left(v, v_{0}\right)=\frac{1}{\pi} \frac{\gamma}{\gamma^{2}+\left(v-v_{0}\right)^{2}}
\end{aligned}
$$

where $A_{e, p k}$ is the peak absorption for the specified peak, $v_{0}$ is the wavenumber of the peak center, $A_{e}(v)$ is the absorption at wavenumber $v, \gamma$ is the linewidth (half-width at half-maximum, in $\left.\mathrm{cm}^{-1}\right)$, and $f\left(v, v_{0}\right)$ is the normalized Lorentz lineshape in $\mathrm{cm}^{-1}$.

Equation (15) is used in a nonlinear fit routine to find the parameters that minimize the root-mean-square error (RMSE). Because of potential errors in our 
frequency scale, we calculate the error in both the frequency and the absorbance axes, $\mathrm{as}^{30}$ :

$$
R M S E=\sqrt{\frac{1}{N} \sum_{i=1}^{N}\left[\frac{1}{\sigma_{A}^{2}}\left(A_{e, i}-A_{e, i}^{\prime}\right)^{2}+\frac{1}{\sigma_{v}^{2}}\left(v_{e, i}-v_{e, i}^{\prime}\right)^{2}\right]}
$$

where the data for the peak are the $i=1 . . N$ points $\left(v_{e, i}, A_{e, i}\right), A_{e, i}^{\prime}$ and $v_{e, i}^{\prime}$ are the estimated absorption of the Lorentizian function, that are closest to each of the datapoints. $v_{e, I}^{\prime}$, and $\sigma_{\mathrm{A}}{ }^{2}$ and $\sigma_{v}{ }^{2}$ are the variances of the absorbance and the frequency, respectively, which normalize the error for each dimension. The fit parameters are the peak height $\left(A_{e, p k}\right)$, the half-width at half-maximum linewidth $(\gamma)$, and the center frequency $\left(v_{0}\right)$. The spectral line intensity, $S\left(\mathrm{~cm}^{-1} /\left(\operatorname{molec} / \mathrm{cm}^{2}\right)\right)$, scaled by the concentration, $C\left(\mathrm{molec} / \mathrm{cm}^{3}\right)$, and the path length, $L(\mathrm{~cm})$, is equal to the integral of the Lorentzian, so that:

$$
C=\left(\frac{\pi}{L S}\right) A_{e, p k} \gamma
$$

Therefore, it is possible to determine the concentration using the spectral line intensities from the HITRAN database, once the peak height and linewidth are determined. However, since the Lorentzian linewidth parameter, $\gamma$, depends on the pressure and temperature, if they are constant then the peak height is proportional to the concentration.

In practice, an absorption spectrum is presented as plot of absorption coefficient as a function of wavenumber. The absorption coefficient, $\alpha$, is related to the absorption 
by $\alpha=\mathrm{A} / \mathrm{L}$, and so we scaled the above results be the length of the cavity. A typical dataset and the corresponding Lorentzian fit is shown in Figure 23, where the data has been fit as described above, and the frequency scale has been determined using the quadratic fit to the applied voltage, as described in Chapter 3. The coefficients are modified slightly from the best fit discussed in 3.3 to provide the best fit to the theoretical Lorentzian. The final fit parameters are within the confidence interval of the best. The theoretical absorption coefficient spectrum was obtained using spectral line intensities, wavenumbers, and linewidths from the HITRAN database ${ }^{1}$, which are then translated to absorption coefficients based on the cell pressure, temperature, and known gas concentration. The peaks shown are $\mathrm{H}_{2} \mathrm{O}$ in ambient lab whole air samples at a measured pressure of 500 torr, a temperature of $22{ }^{\circ} \mathrm{C}$, and an $\mathrm{H}_{2} \mathrm{O}$ concentration of 13.0 parts-perthousand, as measured using a LI-840 non-dispersive infrared (NDIR) $\mathrm{CO}_{2} / \mathrm{H}_{2} \mathrm{O}$ analyzer (LI-COR, Inc., see Section 6.5). The larger and smaller peak are centered at $7640.764 \mathrm{~cm}^{-}$ ${ }^{1}$ and $7640.565 \mathrm{~cm}^{-1}$, respectively ${ }^{1}$. These $\mathrm{H}_{2} \mathrm{O}$ peaks (which are part of the $2 v_{1}$ and $v_{1}+2 v_{2}$ vibrational overtone bands, respectively) were modeled following the procedure outlined in the Rothman et.al. ${ }^{83}$ The data matches well for both confirming that these are the peaks measured by the CRDS, and that the CRDS is functioning properly. The error between the peak heights for the Lorentzian fit to the data $\left(2.58 \times 10^{-6} \mathrm{~cm}^{-1}\right)$ and the theoretical peak height $\left(2.67 \times 10^{-6} \mathrm{~cm}^{-1}\right)$ is $9 \times 10^{-8} \mathrm{~cm}^{-1}$. Using this peak height to estimate the concentration, the CRDS-measured $\mathrm{H}_{2} \mathrm{O}$ concentration would be 12.5 partsper-thousand, which is a 3.3\% error. Since the LI- 840 has a specified accuracy of $1.5 \%$, this suggests a comparable accuracy for the CRDS system. Calibration of $\mathrm{H}_{2} \mathrm{O}$ 
concentration using a dewpoint generator would improve accuracy of measurements made using this system.

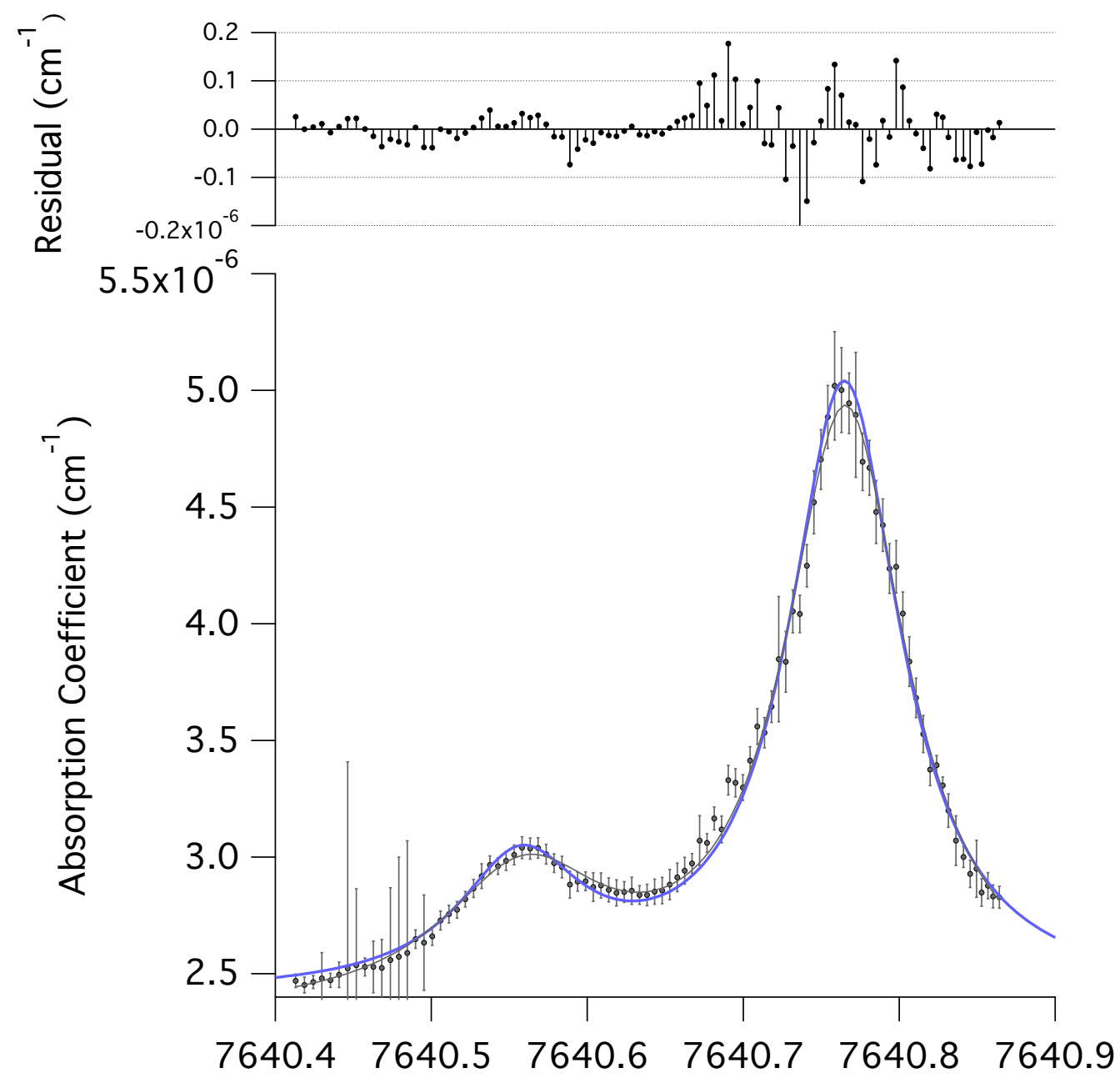

Figure 23 Plot of the v CRDS output (black dots), the Lorentzian fit (gray line), and the expected spectrum using the HITRAN database (blue line). To get the CRDS output wavenumber, the output voltage was scaled by following: $v(\mathrm{~V})=-0.009 \mathrm{~V}^{2}-0.127 \mathrm{~V}+7640.887$, where these coefficients are well within the error limits of the $v(V)$ curve fit in Section 2.3. The residuals from the Lorentzian fit (gray line) are shown in the inset.

It can be seen in Figure 23 that ringdowns were not recorded for certain modes, so there are gaps in the data points across the spectrum. This was most likely a result of issues with the optical feedback scheme, so that the laser never successfully locked onto 
that cavity mode. Small differences between the laser-cavity distance and the cavity length may be the cause. This is discussed further in Section 6.7.

\subsection{Precision and Limit of Detection}

To consider the performance of the system, we look first at the precision. The most base-level precision we can evaluate is the precision of a single absorption coefficient measurement, $\alpha$, which is the fractional absorption loss per unit length, in units of $\mathrm{cm}^{-1}$. These are the typical statistics generated during the measurements at a single cavity mode and frequency over a $100 \mathrm{~ms}$. We base our precision on the $95 \%$ confidence interval, which indicates the range around the measured $\alpha$ within which the true value will be $95 \%$ of the time. If $\bar{\alpha}$ is the average, then the range is given by $\left(\bar{\alpha}-\delta_{\alpha, 95}, \bar{\alpha}+\delta_{\alpha, 95}\right)$ where $\delta_{\alpha, 95}$, is what we call our precision, and is proportional to the standard error of the mean $\left(\sigma_{\alpha} / \sqrt{N}\right)^{73}$ :

$$
\delta_{\alpha, 95}=1.96 * \frac{\sigma_{\alpha}}{\sqrt{N}}
$$

Here $\sigma_{\alpha}$ is the standard deviation associated with $\bar{\alpha}$, and $N$ is the number of decay constants in the average, and for a normal distribution, $95 \%$ of the samples will be within \pm 1.96 standard deviations. In our system, looking at the average precision for a typical spectra, we had an average standard deviation of $7 \times 10^{-8} \mathrm{~cm}^{-1}$, yielding an average $\delta_{\alpha, 95}$ $=1.2 \times 10^{-9} \mathrm{~cm}^{-1}$. If we restrict ourselves to the baseline, in which the standard deviation is lower, $\delta_{\alpha, 95}$ becomes $9 \times 10^{-10} \mathrm{~cm}^{-1}$. The precision at the peak is slightly larger, with 
$\delta_{\alpha, 95}=3.27 \times 10^{-9} \mathrm{~cm}^{-1}$, and is related to the larger variation in each measurement (average standard deviation of $1.2 \times 10^{-7} \mathrm{~cm}^{-1}$ ). These measures are of the system's current optimal performance.

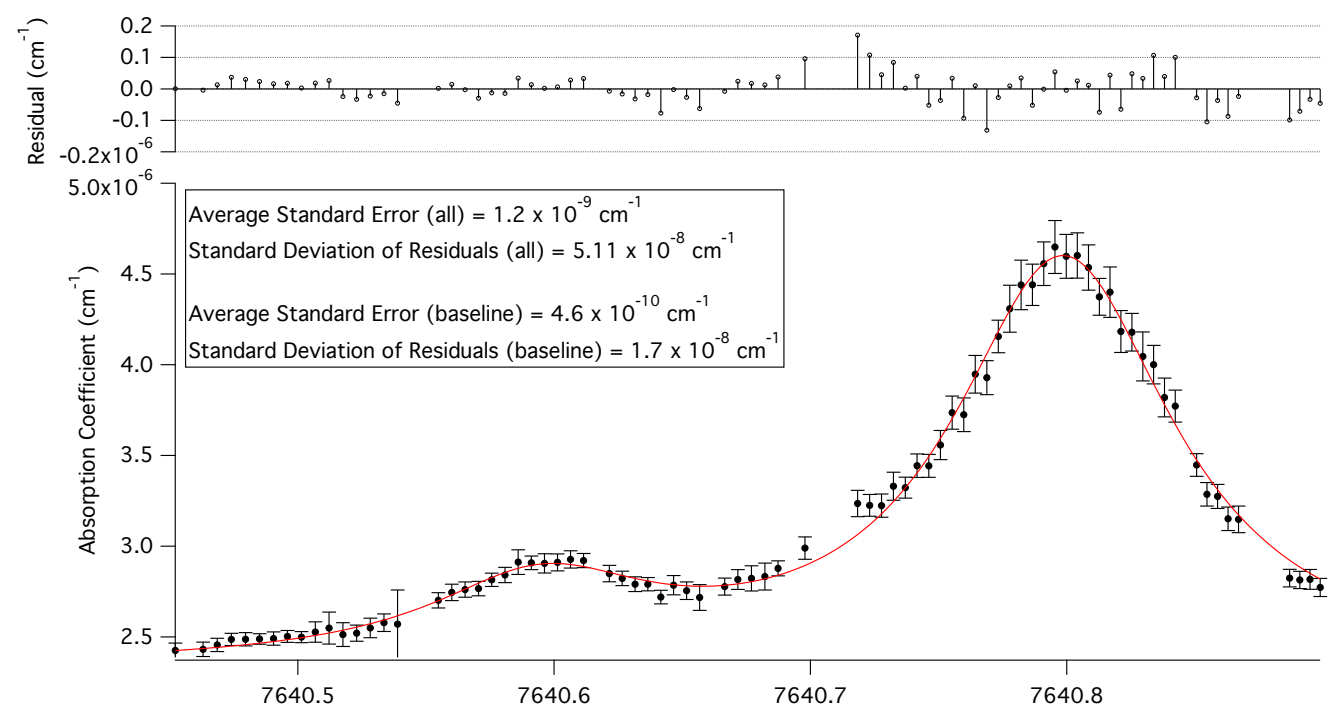

Figure $24 \mathrm{H}_{2} \mathrm{O}$ spectra including the Lorentzian fit and the residual. We can estimate precision $\delta_{\alpha .95}$, by multiplying the standard error by 1.96 .

We can also look at the statistics of multiple absorption measurements taken consecutively at the same frequency, to provide more information about the precision of the system over longer periods ( $\sim \mathrm{min})$. We collected a series of data at a constant laser current/frequency-i.e., the laser's applied voltage was constant while the system was run. Therefore the data was collected in 100 ms groups, after a ringdown was collected, as described in the code description. Figure 25 shows the average absorption coefficients for each block, with error bars indicating the one standard deviation. Note that typical variation between blocks is on the order of the standard deviation of the data points. 
Figure 26 shows a histogram of the 5682 ringdowns with a nearly Gaussian distribution, but a slight skew towards lower absorption. If we look at the precision of these measurements as a whole, we get $\delta_{\alpha .95}=1.6 \times 10^{-8} \mathrm{~cm}^{-1}$, which is an order of magnitude larger than that of the baseline in Figure 24 and the average precision of the individual measurements of $2.2 \times 10^{-8} \mathrm{~cm}^{-1}$. This suggests that over minute timescales, the variation of the absorption coefficient varies, and the negative skew can be attributed to the fact that a linear fit showed that over the entire sample set their was a net drift towards lower absorption of $-7 \times 10^{-8} \mathrm{~cm}^{-1}$.

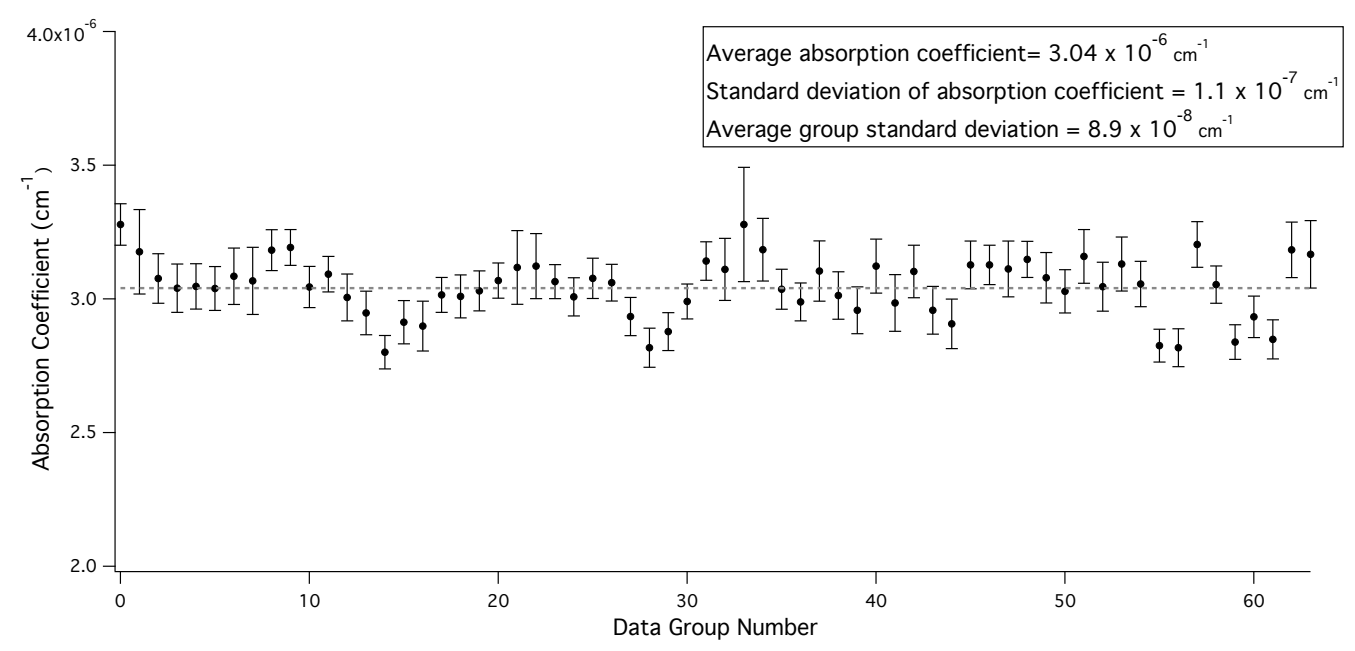

Figure 25 Repeated measurements of absorption coefficient at a constant laser current. The program collected data repeatedly in $100 \mathrm{~ms}$ blocks. The graph shows the average for each $100 \mathrm{~ms}$ period, with the error bars indicating the $95 \%$ confidence interval. The dashed line shows the overall average absorption coefficient for the 64 periods. 


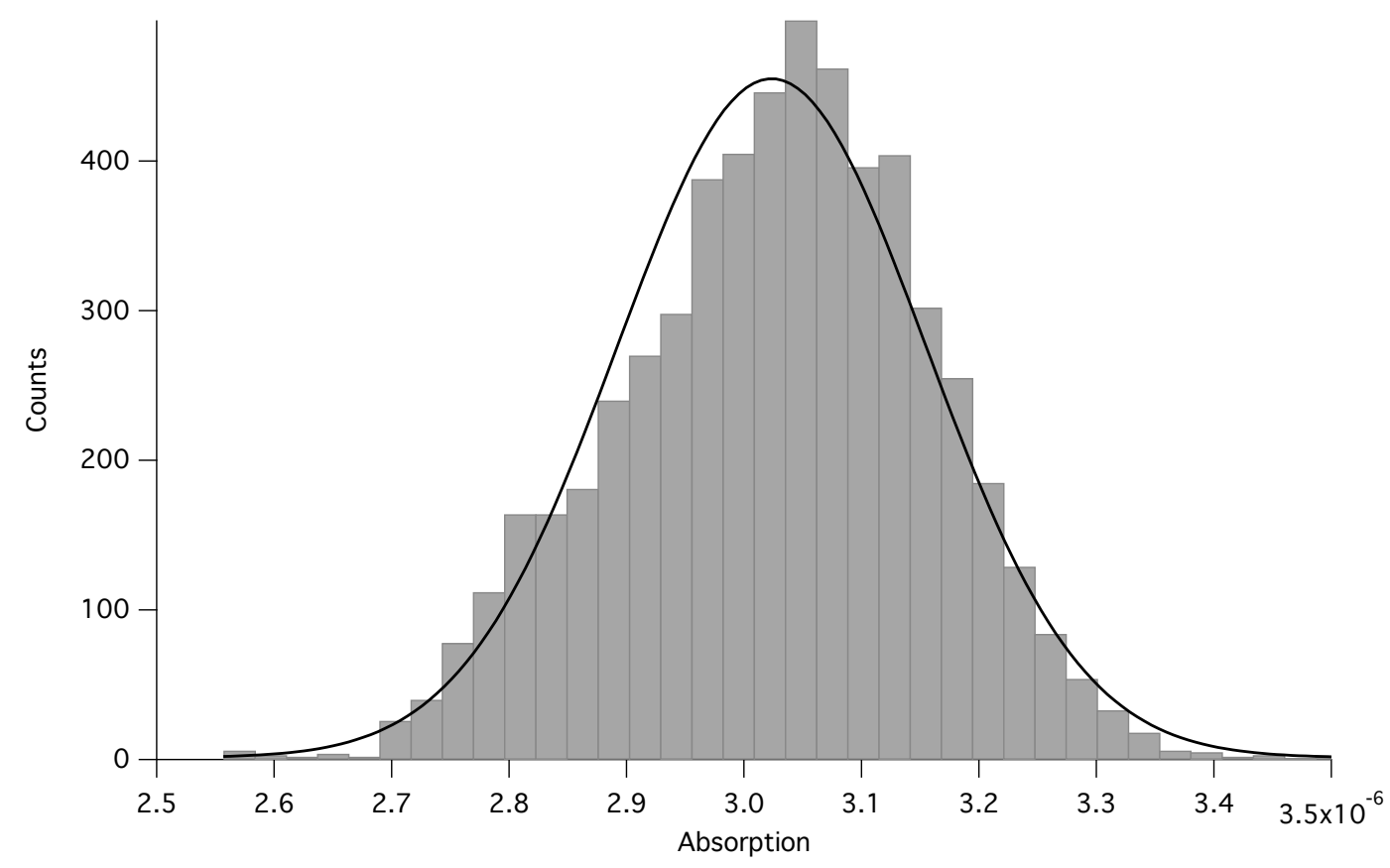

Figure 26 Histogram of the 5682 absorption measurements taken at a constant laser current. Also shown is a fit for a Gaussian distribution. Statistics indicate that in this case, the relative standard deviation is $4.5 \%$, and the precision is $1.6 \times 10^{-8} \mathrm{~cm}^{-1}$.

To address this issue, we looked at a plot of the Allan Variance ${ }^{84}$ for the data set, which looks at the average two-point variance $\left(\sigma^{2}\right)$ between two non-adjacent data points, with increasing spacing between the points It provides an indication of both the stability of the system and the optimal number of averages to use for a measurement (to minimize the uncertainty). The Allan Variance plot for the dataset is shown in Figure 27, and the Allan Variance decreases up to $\sim \mathrm{N}=100$ but increases after about 200 samples, suggesting longer timescale drift in the decay constant. Since these measurements were taken in 100 ms blocks, with a time lag in between, it also suggests that there is significant variation between averaged data points. The minimum Allan Variance of $2 \mathrm{x}$ $10^{-12}\left(\mathrm{~cm}^{-1}\right)^{2}$ at $\mathrm{N}=200$, has a corresponding $\delta_{\alpha, 95}$ of $4.2 \times 10^{-10} \mathrm{~cm}^{-1}$, consistent with our baseline precision determined above. 


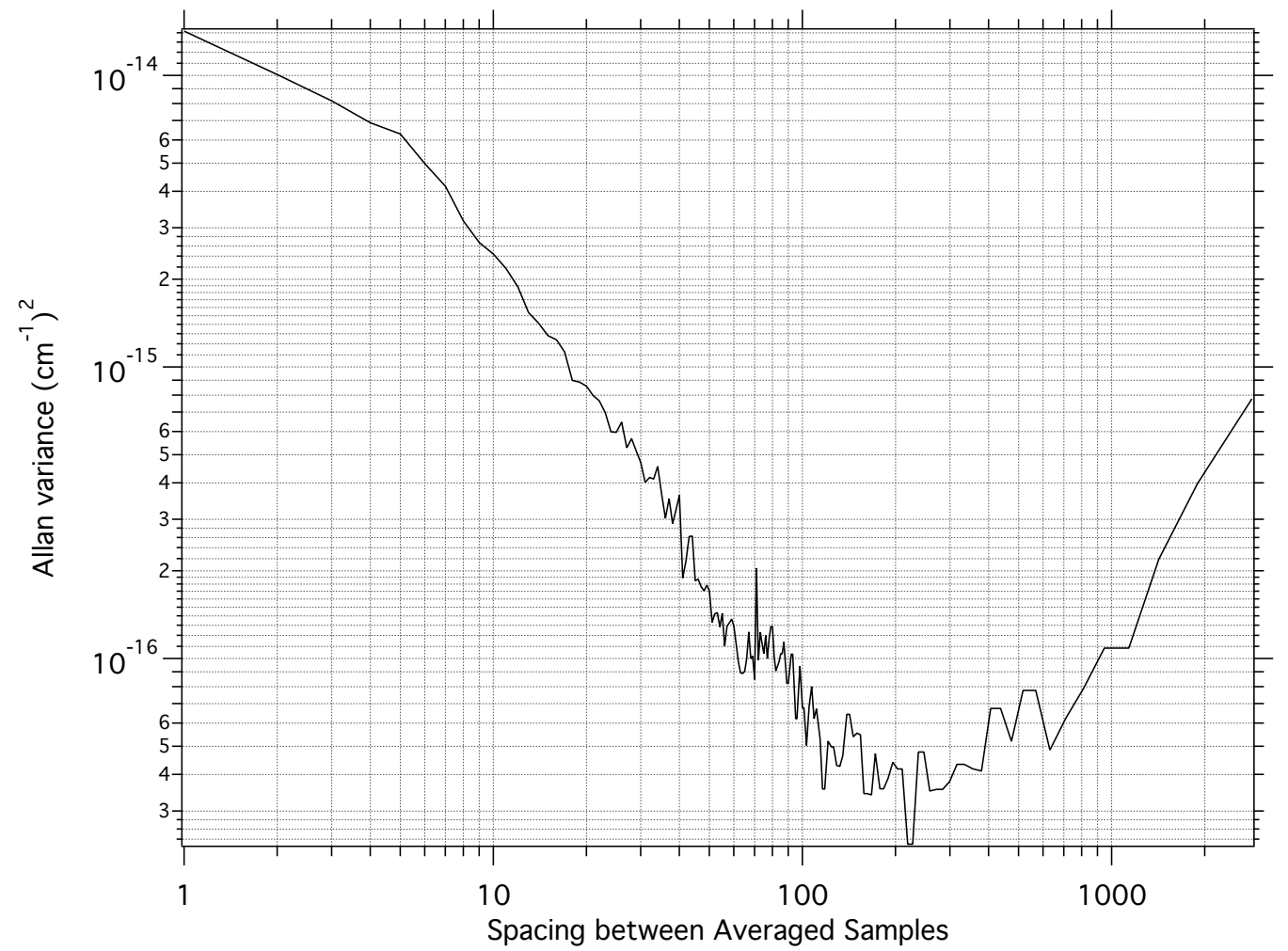

Figure 27 Allan Variance of repeatability data, which suggests that the sensitivity of the overall system is best when the number of averages is 200-300 data points, or approximately $300 \mathrm{~ms}$. This could be a result of the maximum number of averages stored being 150, such that over time the variation increases.

Despite a reasonable precision of $3.3 \times 10^{-9} \mathrm{~cm}^{-1}$ for an individual data point, our system appears to exhibit instabilities in repeated measurements that significantly reduce the overall precision by an order of magnitude when considered across multiple points. We would therefore expect that this would affect concentration measurements based on a Lorentzian fit of multiple points and in numerous repeated scans.

We next considered the sensitivity, or minimum detectable absorption level (MDAL), by looking at the average standard deviation of the residuals of the baseline, similar to that done by Motto-Ros ${ }^{41}$, and others mentioned in Section 1.4. Figure 28 shows another typical spectrum, where in this case we have restricted our view to the smaller $\mathrm{H}_{2} \mathrm{O}$ peak. The Lorentzian fit to the data is also shown. We approximated our 
baseline to be to the left of the smaller peak (lower in wavenumber), and the residuals for the fit are shown in the inset, with an rms value of $2.1 \times 10^{-8} \mathrm{~cm}^{-1}$. This result is consistent with our expectation based on the variation of multiple measurements to be 1.6 $\mathrm{x} 10^{-8} \mathrm{~cm}^{-1}$.

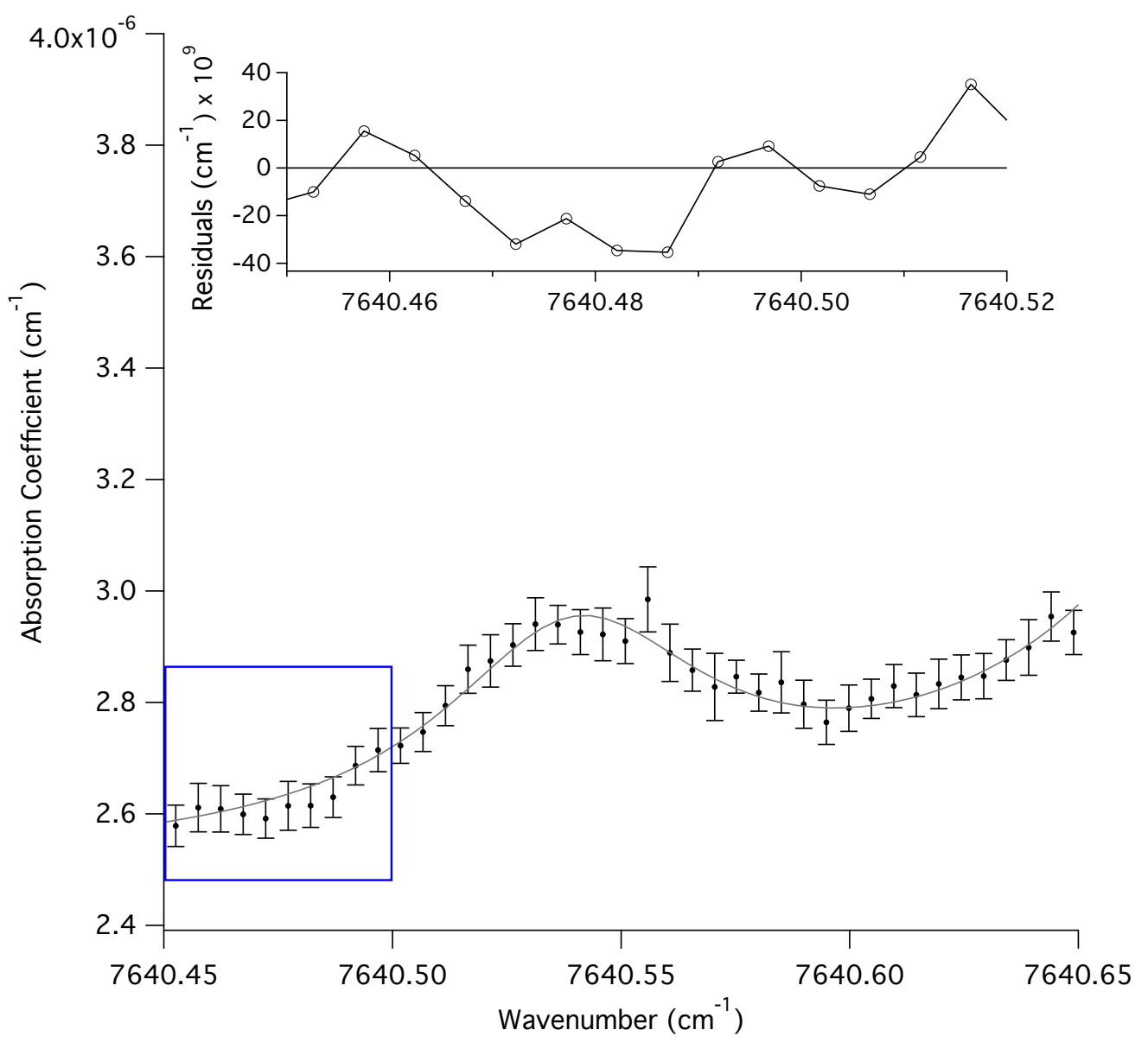

Figure 28 Estimating the limit of detection of the system. Using the standard deviation of the residuals at the baseline (shown in the inset). In this case, we chose the region to the left of the smaller peak (enclosed in the blue box) to represent the baseline.

Also of note is the fact that at the larger peak, the mean standard deviation is noticeably larger than the baseline (as in Figure 23 and Figure 24), and we might expect this to have an impact on the residuals. It can also be seen that the data does not follow 
the Lorenztian line fit as closely as it does on the lower peak or at the baseline. In fact the average rms of the residuals at the peak is $9 \times 10^{-8} \mathrm{~cm}^{-1}$.

\subsection{Concentration measurement repeatability and linearity}

Now, having looked at the precision and detection limits based on a single and multiple data points, we then examined the precision of the system in its ability to measure gas concentration, specifically $\mathrm{H}_{2} \mathrm{O}$. Since the peak height is proportional to the concentration, to determine our system's response, we simply looked at the peak height for the largest peak of the Lorentzian fit to our data (like those shown in Figure 23 and Figure 24). To study this, we needed an independent measure of $\mathrm{H}_{2} \mathrm{O}$ concentrations. Using our vacuum system we drew in lab air into the CRDS, while simultaneously drawing air into an LI-840 $\mathrm{CO}_{2} / \mathrm{H}_{2} \mathrm{O}$ gas analyzer (LI-COR, Inc., Lincoln, Nebraska). The LI-840 is an NDIR gas analyzer, which uses a $14-\mathrm{cm}$ cavity for single-pass broadband infrared absorption measurement, in the $4000 \mathrm{~nm}$ infrared region. For ambient $\mathrm{H}_{2} \mathrm{O}$, it has a range of 0-80 parts-per-thousand, an accuracy of $1.5 \%$, and an RMS noise of 0.07 parts-per-thousand. In our setup, it was configured for 20-second signal averaging, and recorded the $\mathrm{H}_{2} \mathrm{O}$ concentration every 2 minutes. Prior to the measurements used here, it was zeroed using a Hydro-Purge II moisture trap (W. R. Grace \& Co., Columbia, Maryland). The LI-840 $\mathrm{H}_{2} \mathrm{O}$ instrument was calibrated previously using a LI-COR (Model LI-610) dew point generator (with an accuracy of $\pm 0.2 \%)$

With this configuration, we compared the response of our system to the $\mathrm{H}_{2} \mathrm{O}$ concentration as measured on the LI-840. Figure 29 shows repeated peak height 
measurements at a nearly constant $\mathrm{H}_{2} \mathrm{O}$ concentration $(9.5 \pm 0.2$ parts-per-thousand, as measured by the LI-840). The average absorption measured by the CRDS system was $2.89 \times 10^{-6} \mathrm{~cm}^{-1}(\mathrm{~N}=50)$, with a relative standard deviation of $2.2 \%$. This is consistent with the variation in the LI-840 measurements, and the corresponding LI-840 data exhibited a small negative slope, also consistent with the CRDS measurements. The precision for the overall peak absorption coefficient was $\pm 1.8 \times 10^{-8} \mathrm{~cm}^{-1}(0.6 \%$ relative precision)

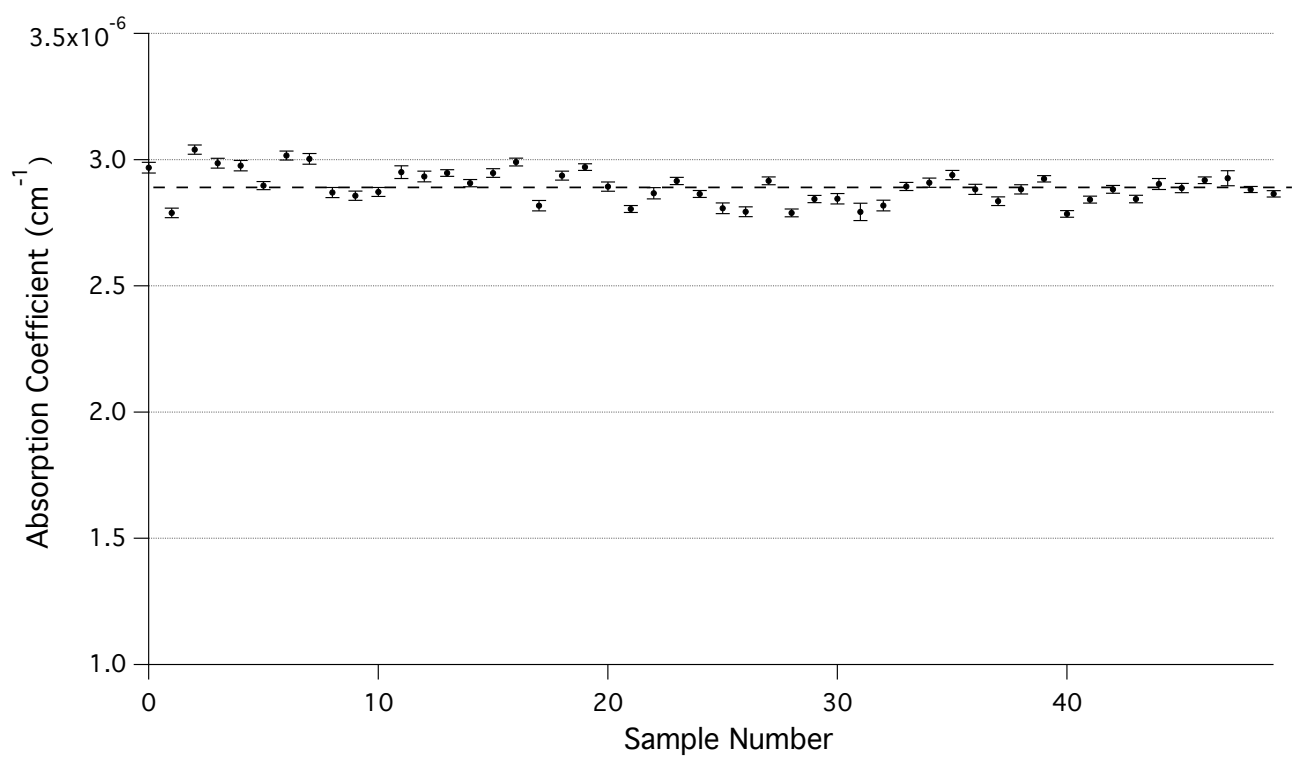

Figure 29 Repeatability of the Lorentzian fit peak height (which is proportional to the concentration). The data shows 50 trials at a nearly constant $\mathrm{H}_{2} \mathrm{O}$ concentration. The statistics show an average absorption of $2.9 \times 10^{-6} \mathrm{~cm}^{-1}$ (shown as the dashed line) and standard deviation of $6.4 \times 10^{-8} \mathrm{~cm}^{-1}$, yielding a relative standard deviation of $2.2 \%$. The error bars indicate the RMSE of the Lorentzian fit for each data concentration measurement.

Finally, we are interested in the linearity of the system, by looking at its response to a varying $\mathrm{H}_{2} \mathrm{O}$ concentration. We set up a system so that both the LI-840 and our CRDS system were sampling the same air, and ran them both continuously overnight. We were fortunate in that the ambient $\mathrm{H}_{2} \mathrm{O}$ concentration changed by almost $50 \%$, from 12 
parts-per-thousand to 7 parts-per-thousand in a 5-hour period. We then compared the peak absorption coefficient of the Lorentzian fit for the CRDS system to the $\mathrm{H}_{2} \mathrm{O}$ concentration reported by the LI-840. A plot showing that comparison is shown in Figure 30. While there was significant variation around the linear fit, the relative standard error in the slope was $0.2 \%$, and the residuals (shown in the inset of Figure 30), had an rms value of $9.4 \times 10^{-8} \mathrm{~cm}^{-1}$, which is approximately a $4 \%$ relative variation.
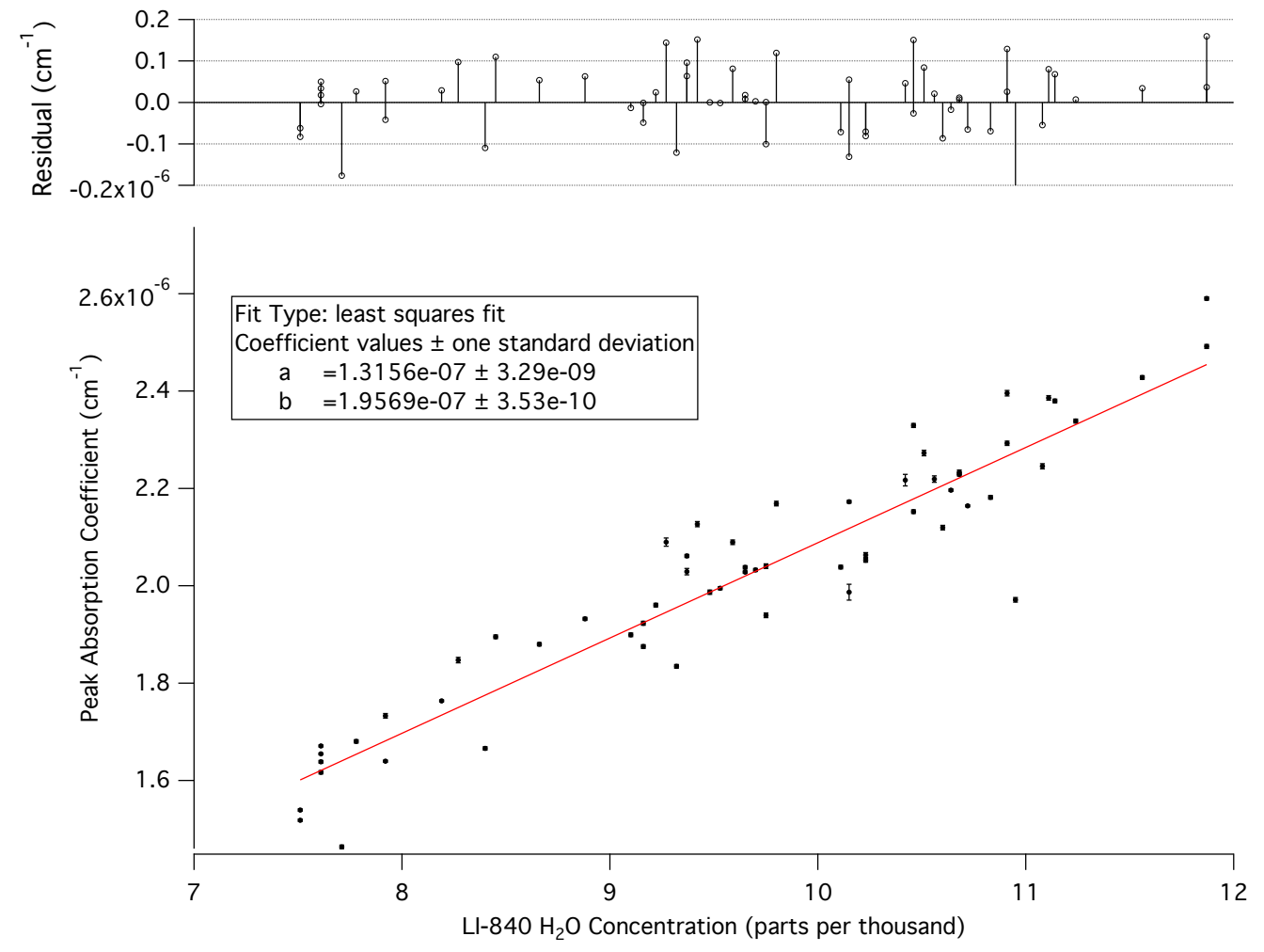

Figure 30 Correlation of CRDS system with LI-840 NDIR measurements. During this trial the LI-840 sampled the same air as the CRDS cavity. A linear fit (red line) between the two shows the CRDS responds to changes in $\mathrm{H}_{2} \mathrm{O}$ concentration linearly. The data was taken over approximately a 5 hour period.

To determine whether the residuals to the linear fit are distributed normally, we used the Q-Q plot in Figure 31, which plots the normalized residuals versus a Gaussian 
distribution. The closer the points follow the 1:1 line, the closer the distribution is to Gaussian. Since the residuals exhibit a near normal variation (except for several outliers at the low end), we can see that the CRDS system's response to $\mathrm{H}_{2} \mathrm{O}$ concentration was linear over this range. To be more rigorous, we also applied the Kolmogorov-Smirnoff test for normality, and found that it is linear within a $95 \%$ confidence interval $(D=0.08<$ $\left.D_{95 \%}=0.23\right)$.

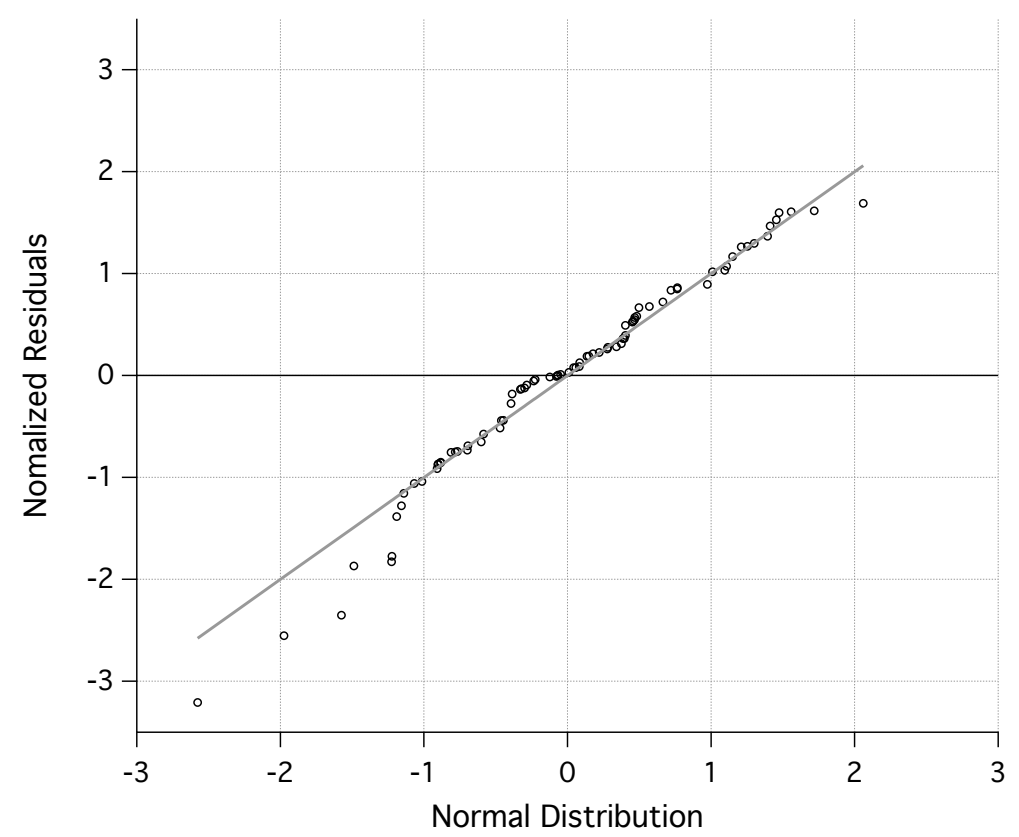

Figure 31 Q-Q plot of residuals for the line fit. The residuals were normalized and plotted against a Gaussian probability distribution (circles). The solid line is a 1:1 line, and deviations of the residuals from this line indicate non-normal distribution. Significantly on the low end there are several outliers that deviate significantly from a Gaussian distribution.

\subsection{Comparison with previous works}

The laser-cavity locking combined with the optical injection unlocking enabled us to achieve ringdown rates of $3.5 \mathrm{kHz}$, for ringdowns with a time constant of $12 \mu \mathrm{s}$. We demonstrated our data acquisition and processing system was capable of processing 
ringdowns to at a rate of $4.4 \mathrm{kHz}$, with a current operational limit of $1.5 \mathrm{kHz}$ transfer rate to the computer.

Comparing these rates to the previous work described in Chapter 1, we see that it exceeds the rate of our previous system, and has the fastest demonstrated ringdown processing rate. It should be reiterated that our rate included individual processing of each ringdown. As mentioned in 1.4, many systems either off-load time-series data for later decay constant determination, or average multiple ringdowns before determining the decay constant. For example, Orr and $\mathrm{He}^{42}$ averaged ringdowns prior to processing and demonstrated the highest acquisition rate of $5 \mathrm{kHz}$. Spence et.al. ${ }^{59}$ claimed their processing system could operate at $1 \mathrm{MHz}$, but it was only demonstrated at a rate of 20 Hz. Motto-Ros et. al. ${ }^{41}$, also demonstrated an effective ringdown capture rate of $640 \mathrm{~Hz}$.

The advantage of our design is that we are able to average significantly more ringdowns per mode before mechanical instabilities introduce significant error, whereas they were limited to 50 averages per mode because their system is designed to sweep across all modes, collecting 1 ringdown per mode, per sweep.

The precision, limit of detection, and variation in the individual decay constants (and subsequent absorption coefficient) of the system was worse than many of the other systems reviewed in Section 1.4. Our demonstrated MDAL of $1.6 \times 10^{-8} \mathrm{~cm}^{-1}$ is $1-2$ orders of magnitude worse than typical systems reviewed. Because of the high acquisition rate, our precision for absorption coefficient at a single mode is respectable, at $3 \times 10^{-9} \mathrm{~cm}^{-1}$. As described in Berden et.al., ${ }^{85}$ the theoretical minimum detectable absorption coefficient is based on the reflectivity of the cavity mirrors, and the accuracy of the ringdown time constant. Based on this our theoretical minimum detectable level is 
$(1-R) \delta_{\alpha .95}(\alpha L)^{-1}$, or $4.2 \times 10^{-9} \mathrm{~cm}^{-1}$ —an order of magnitude lower than our measured detection limit.

\subsection{System improvements}

Despite the 2 order of magnitude increase in ringdown acquisition rate resulting from our novel advances in laser switching and ringdown data processing, the overall CRDS system still lacks precision. The most likely cause is the performance of the linear optical feedback design, resulting from the interaction between the laser-cavity path resonance and the cavity resonance. This also caused some missed resonance modes of the cavity and difficulty in laser locking. It is also suspected for causing a variation in the cavity output that did not vary linearly with laser current, which made finding an optimal trigger level for the system problematic. Finally, optical feedback performance may also be responsible for reduced numbers of ringdowns as the absorption of the analyte increased in some spectra. Further evidence is the variation of decay constants being on the order of $1 \%$, which is significantly larger than that measured on other systems that use laser-cavity locking ${ }^{2,41}$.

While it is expected that maintaining the linear optical feedback design and optimizing the laser-cavity distance to better match the cavity length could reduce these effects and improve the stability, converting the cavity to a V-cavity design like that of Morville et.al. ${ }^{54}$, could dramatically improve the laser-cavity locking, and in so doing dramatically improve the precision of the system. Furthermore, it likely that due to the non-Gaussian beamshape of the laser that during the frequency sweep additional cavity modes are being excited (causing energy to be built up at a non-TEM $\mathrm{To}_{00}$ mode. If the 
buildup is sufficient to trigger the pulsed laser, then the background decay constant could differ enough to affect the precision. Therefore, including additional optics to improve beam quality may also have an impact on the precision of the system.

Additional improvements could be made in the FPGA acquisition system by (1) expanding the number of bits recorded to improve the resolution and dynamic range of the system, (2) adding the ability to average several components of the FFT on the FPGA to reduce the variation of individual decay constant estimates ${ }^{70}$, (3) use the FPGA to average the collected time constants, so that the ringdown acquisition rate is no longer limited by the memory on the Arduino Due, since only the average decay constant would need to be sent to the computer. This would also aid in improving the precision as more decay constants could be averaged, and (4) use the USB interface on the FPGA to store and transmit the values to the computer, avoiding the Arduino Due altogether. These last two would also enable a more compact acquisition and control system, as it would further reduce the computational power required on the computer. It would also increase data acquisition rates close to the theoretical maximum of $3.5 \mathrm{kHz}$. One possibility is to use an inexpensive single-board computer like the Raspberry Pi, which easily interfaces with Arduino microcontrollers, and provides a user interface.

Once the optical stability has been improved, addressing mechanical factors can make improvements in the precision of CRDS systems. Tight control of the cavity temperature and pressure are important for the repeatability of gas concentration measurements because they both affect shape of the spectral lines. The precision and repeatability of the Picarro, Inc. CRDS systems, for example, was achieved by maintaining small tolerances on the cavity pressure and temperature. This ensures the 
lineshape remains the same, allowing them to determine gas concentrations by only measuring the peak height and a baseline ${ }^{35}$.

Finally, the methods developed here are applicable to any wavelength. The line intensities of both $\mathrm{H}_{2} \mathrm{O}$ and $\mathrm{CH}_{4}$ are significantly lower in the $1309 \mathrm{~nm}$ region of the near IR because the absorption are due to third overtones, and the lines measured here had line intensities on the order of $10^{-24}\left(\mathrm{~cm}^{-2}\right.$ molecules $\left.{ }^{-1}\right) \mathrm{cm}^{-1}$. Having now demonstrated the techniques in the near-IR, shifting to other regions would significantly increase the sensitivity of the design to gas concentrations. Indeed, staying in the near-IR and shifting near $1650 \mathrm{~nm}$ to a second overtone of $\mathrm{CH}_{4}$ would increase the line intensities by 3 orders of magnitude ${ }^{17}$. Converting the system to this range would, however, require new lasers, detectors, cavity mirrors, and polarization optics. 


\section{Chapter 7 Conclusion}

We have demonstrated a CRDS system with a ringdown acquisition rate of 1.5 $\mathrm{kHz}$, with a maximum capability of $3.5 \mathrm{kHz}$, using new techniques that significantly change the way in which the ringdowns are initiated and processed. We implemented a linear optical feedback scheme allowing the laser to stay on-resonance with the cavity for up to hundreds of milliseconds rather than tenths of a millisecond. We combined this with a novel optical technique for switching the laser off-resonance so that a ringdown could be initiated, but brought the laser back to resonance to allow for another ringdown on the same mode. This allowed us to generate ringdowns on a single cavity mode at a maximum rate of $3.5 \mathrm{kHz}$. Another significant modification was the development of an FGPA-based data acquisition and processing system which determines the time constant at a maximum rate of $4.4 \mathrm{kHz}$, but the current implementation was limited to $1.5 \mathrm{kHz}$ by the Arduino Due that stored and transmitted the time constants to the computer.

The entire system showed promise as we demonstrated the ability to determine gas concentrations using $\mathrm{H}_{2} \mathrm{O}$, and the system measured the concentration with an accuracy of $3.3 \%$. Our system achieved an absorption coefficient precision of $0.1 \%(95 \%$ confidence interval). It also exhibited a linear response for varying $\mathrm{H}_{2} \mathrm{O}$ concentrations, and a $2.2 \%$ variation $(1 \sigma)$ for repeated measurements at the same $\mathrm{H}_{2} \mathrm{O}$ concentration, a corresponding precision of $0.6 \%$, and a limit of detection of $1.6 \times 10^{-8} \mathrm{~cm}^{-1}$. 


\section{References}

${ }^{1}$ L.S. Rothman, D. Jacquemart, A. Barbe, D. Chris Benner, M. Birk, L.R. Brown, M.R. Carleer, C. Chackerian, K. Chance, L.H. Coudert, and others, Journal of Quantitative Spectroscopy and Radiative Transfer 96, 139 (2005).

2 B.A. Paldus and A.A. Kachanov, Canadian Journal of Physics 83, 975 (2005).

${ }^{3}$ A. O'Keefe and D.A.G. Deacon, Review of Scientific Instruments 59, 2544 (1988).

${ }^{4}$ R. Engeln, G. Berden, R. Peeters, and G. Meijer, Review of Scientific Instruments 69, 3763 (1998).

${ }^{5}$ A. O’Keefe, J.J. Scherer, and J.B. Paul, Chemical Physics Letters 307, 343 (1999).

${ }^{6}$ D. Herriott, H. Kogelnik, and R. Kompfner, Appl. Opt. 3, 523 (1964).

${ }^{7}$ J.U. White, J. Opt. Soc. Am. 66, 411 (1976).

${ }^{8}$ A.C. Stanton and J.A. Silver, Appl. Opt. 27, 5009 (1988).

9 J.T. Hodges, H.P. Layer, W.W. Miller, and G.E. Scace, Review of Scientific Instruments 75, 849 (2004).

${ }^{10}$ D. Romanini and K.K. Lehmann, The Journal of Chemical Physics 99, 6287 (1993).

${ }^{11}$ H. Dahnke, D. Kleine, P. Hering, and M. Mürtz, Appl Phys B 72, 971 (2001).

${ }^{12}$ D.B. Atkinson and J.L. Spillman, J. Phys. Chem. A 106, 8891 (2002).

${ }^{13}$ J. Pearson, A.J. Orr-Ewing, M.N.R. Ashfold, and R.N. Dixon, The Journal of Chemical Physics 106, 5850 (1997).

${ }^{14}$ L. Zhu, D. Kellis, and C.-F. Ding, Chemical Physics Letters 257, 487 (1996).

${ }^{15}$ D.B. Atkinson and J.W. Hudgens, J. Phys. Chem. A 103, 4242 (1999).

${ }^{16}$ C.S. Moreau, E. Therssen, X. Mercier, J.F. Pauwels, and P. Desgroux, Appl Phys B 78, 485 (2004).

${ }^{17}$ B.L. Fawcett, A.M. Parkes, D.E. Shallcross, and A.J. Orr-Ewing, Physical Chemistry Chemical Physics 4, 5960 (2002).

18 J.C. Turnbull, A. Karion, M.L. Fischer, I. Faloona, T. Guilderson, S.J. Lehman, B.R. Miller, J.B. Miller, S. Montzka, T. Sherwood, S. Saripalli, C. Sweeney, and P.P. Tans, Atmos. Chem. Phys. 11, 705 (2011).

19 O.J. Maselli, D. Fritzsche, L. Layman, J.R. McConnell, and H. Meyer, Isotopes in Environmental and Health Studies 49, 387 (2013).

${ }^{20}$ M. Mazurenka, R. Wada, A.J.L. Shillings, T.J.A. Butler, J.M. Beames, and A.J. OrrEwing, Applied Physics B: Lasers and Optics 81, 135 (2005).

${ }^{21}$ M. Bitter, S.M. Ball, I.M. Povey, and R.L. Jones, Atmos. Chem. Phys. 5, 2547 (2005).

${ }^{22}$ D.B. Atkinson, J.G. Radney, and J.W. Harworth, AGU Fall Meeting Abstracts -1, 06 (2010).

${ }^{23}$ T.J.A. Butler, J.L. Miller, and A.J. Orr-Ewing, The Journal of Chemical Physics 126, 174302 (2007).

${ }^{24}$ D.B. Atkinson, J.G. Radney, and M.E. Wright, AGU Fall Meeting Abstracts -1, 1426 (2007).

${ }^{25}$ M. Ueyama, K. Hamotani, W. Nishimura, Y. Takahashi, N. Saigusa, and R. Ide, Theor Appl Climatol 109, 461 (2012). 
26 P. Bergamaschi, M. Brläunlich, T. Marik, and C.A.. Brenninkmeijer, Journal of Geophysical Research-Atmospheres 105, (n.d.).

${ }^{27}$ G.W. Santoni, B.C. Daube, E.A. Kort, R. Jiménez, S. Park, J.V. Pittman, E. Gottlieb, B. Xiang, M.S. Zahniser, D.D. Nelson, J.B. McManus, J. Peischl, T.B. Ryerson, J.S. Holloway, A.E. Andrews, C. Sweeney, B. Hall, E.J. Hintsa, F.L. Moore, J.W. Elkins, D.F. Hurst, B.B. Stephens, J. Bent, and S.C. Wofsy, Atmos. Meas. Tech. 7, 1509 (2014).

${ }^{28}$ H. Dahnke, D. Kleine, W. Urban, P. Hering, and M. M\ürtz, Applied Physics B: Lasers and Optics 72, 121 (2001).

${ }^{29}$ Y. Chen, K.K. Lehmann, J. Kessler, B.S. Lollar, G.L. Couloume, and T.C. Onstott, Anal. Chem. 85, 11250 (2013).

${ }^{30}$ G. Bostrom, Development of a Cavity Ring-Down Spectroscopic Technique for Measuring Stable Isotopes in Atmospheric Methane, Portland State University, 2010.

${ }^{31}$ M. Schoell, Geochimica et Cosmochimica Acta 44, 649 (1980).

${ }^{32}$ B.J. Peterson and B. Fry, Annual Review of Ecology and Systematics 18, 293 (1987).

33 A.L. Rice, A.A. Gotoh, H.O. Ajie, and S.C. Tyler, Anal. Chem 73, 4104 (2001).

${ }^{34}$ A.L. Rice and P.D. Quay, Anal. Chem 78, 6320 (2006).

${ }^{35}$ E.R. Crosson, Appl. Phys. B 92, 403 (2008).

${ }^{36}$ H. Chen, J. Winderlich, C. Gerbig, A. Hoefer, C.W. Rella, E.R. Crosson, A.D. Van Pelt, J. Steinbach, O. Kolle, V. Beck, B.C. Daube, E.W. Gottlieb, V.Y. Chow, G.W. Santoni, and S.C. Wofsy, Atmos. Meas. Tech. 3, 375 (2010).

37 T.B. Coplen, Reference and Intercomparison Materials for Stable Isotopes of Light Elements 825, 31 (1995).

38 R. Gonfiantini, W. Stichler, and K. Rozanski, Reference Materials and Intercomparison Materials Distributed by the International Atomic Energy Agency for Stable Isotope Measurements In, Reference and Intercomparison Materials for Stable Isotopes of Light Elements: Vienna, International Atomic Energy Agency (IAEA-TECDOC-825, 1995).

${ }^{39}$ D. Romanini, A.A. Kachanov, N. Sadeghi, and F. Stoeckel, Chemical Physics Letters 264, 316 (1997).

${ }^{40}$ B. Paldus, C. Harb, T. Spence, B. Wilke, J. Xie, J. Harris, and R. Zare, Journal of Applied Physics 83, 3991 (1998).

${ }^{41}$ V. Motto-Ros, J. Morville, and P. Rairoux, Applied Physics B: Lasers and Optics 87, 531 (2007).

42 B.J. Orr and Y. He, Chemical Physics Letters 512, 1 (2011).

${ }^{43}$ Y. He and B.J. Orr, Appl Phys B 75, 267 (2002).

${ }^{44}$ D.A. Long, A.J. Fleisher, S. Wójtewicz, and J.T. Hodges, Appl. Phys. B 115, 149 (2014).

${ }^{45}$ D.B. Atkinson, The Analyst 128, 117 (2003).

${ }^{46}$ G. Bostrom, A. Rice, and D. Atkinson, Opt. Lett. 39, 4227 (2014).

${ }^{47}$ R. Drever, J. Hall, F. Kowalski, J. Hough, G. Ford, A. Munley, and H. Ward, Applied Physics B: Lasers and Optics 31, 97 (1983).

${ }^{48}$ P. Laurent, A. Clairon, and C. Breant, IEEE Journal of Quantum Electronics 25, 1131 (1989).

${ }^{49}$ B. Dahmani, L. Hollberg, and R. Drullinger, Optics Letters 12, 876 (1987). 
${ }^{50}$ D.M. Kane and K.A. Shore, Unlocking Dynamical Diversity: Optical Feedback Effects on Semiconductor Lasers (John Wiley \& Sons Inc, 2005).

${ }^{51}$ J. Ohtsubo, Semiconductor Lasers: Stability, Instability and Chaos (Springer, 2008).

${ }^{52}$ R. Lang and K. Kobayashi, IEEE Journal of Quantum Electronics 16, 347 (1980).

${ }^{53}$ M.G. Littman and H.J. Metcalf, Applied Optics 17, 2224 (1978).

${ }^{54}$ J. Morville, S. Kassi, M. Chenevier, and D. Romanini, Applied Physics B: Lasers and Optics 80, 1027 (2005).

${ }^{55}$ 20-Bit, Low-Power Digital-to-Analog Converter, SBASO82G (Texas Instruments Inc., 2009).

${ }^{56}$ B. Paldus, J. Harris Jr, J. Martin, J. Xie, and R. Zare, Journal of Applied Physics 82, 3199 (1997).

${ }^{57}$ N.J. van Leeuwen, J.C. Diettrich, and A.C. Wilson, Applied Optics 42, 3670 (2003).

${ }^{58}$ J.W. Hahn, Y.S. Yoo, J.Y. Lee, J.W. Kim, and H.-W. Lee, Appl. Opt. 38, 1859 (1999).

59 T.G. Spence, M.E. Calzada, H.M. Gardner, E. Leefe, H.B. Fontenot, L. Gilevicius, R.W. Hartsock, T.K. Boyson, and C.C. Harb, Optics Express 20, 8804 (2012).

${ }^{60}$ L.W.H.R.. Fox, C.W. Oates, and L.W. Hollberg, Cavity-Enhanced Spectroscopies: Experimental Methods in the Physical Sciences, Chapter Stabilizing Diode Lasers to High-Finesse Cavities (Academic Press, 2002).

${ }^{61}$ G.H.M. Van Tartwijk and D. Lenstra, Quantum and Semiclassical Optics: Journal of the European Optical Society Part B 7, 87 (1995).

${ }^{62}$ B.R. Bennett, R.A. Soref, and J.A. del Alamo, IEEE Journal of Quantum Electronics 26, 113 (1990).

${ }^{63}$ W. Kowalsky and K.J. Ebeling, Opt. Lett. 12, 1053 (1987).

${ }^{64}$ K. Ishida, H. Nakamura, H. Matsumura, T. Kadoi, and H. Inoue, Applied Physics Letters 50, 141 (1987).

${ }^{65}$ LM 555 Datasheet (Texas Instruments, Inc., 2000).

${ }^{66}$ M. Gallant, Pulse Circuits for Infrared LEDs and Visible Diode Lasers (2009).

${ }^{67}$ G.H.M. Van Tartwijk, G. Muijres, D. Lenstra, M.P. Van Exter, and J.P. Woerdman, Electronics Letters 29, 137 (1993).

${ }^{68}$ J. Manning, R. Olshansky, and C.B. Su, IEEE Journal of Quantum Electronics 19, 1525 (1983).

${ }^{69}$ M. Mazurenka, R. Wada, A. Shillings, T. Butler, J. Beames, and A. Orr-Ewing, Applied Physics B: Lasers and Optics 81, 135 (2005).

${ }^{70}$ M.A. Everest and D.B. Atkinson, Review of Scientific Instruments 79, 023108 (2008).

${ }^{71}$ T.G. Spence, C.C. Harb, B.A. Paldus, R.N. Zare, B. Willke, and R.L. Byer, Review of Scientific Instruments 71, 347 (2000).

72 R.E. Ziemer, Principles of Communication: Systems, Modulation, and Noise, 5th ed (Wiley, New York, 2002).

${ }^{73}$ A.M. Mood, in 1st ed. (McGraw-Hill, New York, 1950).

${ }^{74}$ K.K. Lehmann and H. Huang, in Frontiers of Molecular Spectroscopy, edited by J. Laane (Elsevier, Amsterdam, 2009), pp. 623-658.

75 R.N. Bracewell, The Fourier Transform and Its Applications, 2nd edition, revised (McGraw Hill, New York, 1986).

${ }^{76} 14-B i t, 125 \mathrm{MSPS} / 105 \mathrm{MSPS} / 80 \mathrm{MSPS}, 1.8 \mathrm{~V}$ Analog-to-Digital Converter, AD9255 Data Sheet, Revision C (Analog Devices, Inc., 2013). 
${ }^{77} 3.3 \mathrm{GHz}$ Ultralow Distortion RF/IF Differential Amplifier, ADL5562, Rev. E (Analog Devices, Inc., 2014).

${ }^{78}$ A.D.I. Engineering, Linear Circuit Design Handbook (Newnes, 2011).

79 J.W. Nilsson and S. Riedel, Electric Circuits, 9 edition (Prentice Hall, Boston, 2010).

${ }^{80}$ LogiCORE IP Fast Fourier Transform v7.1 Product Specification, DS260 (Xilinx, Inc., 2011).

${ }^{81}$ LogiCORE IP Divider Generator v3.0 Product Specification, DS530 (Xilinx, Inc., 2011).

${ }^{82}$ Evaluation Board User Guide: Evaluating the AD9265/AD9255 Analog-to-Digital Converters, UG-074 (Analog Devices, Inc., 2011).

${ }^{83}$ L. Rothman, C. Rinsland, A. Goldman, S. Massie, D. Edwards, J. Flaud, A. Perrin, C. Camy-Peyret, V. Dana, J. Mandin, and others, J. Quant. Spectrosc. Radiat. Transfer 60, 665 (1996).

${ }^{84}$ IEEE Std 1139-1999 0_1 (1999).

${ }^{85}$ G. Berden, R. Peeters, and G. Meijer, International Reviews in Physical Chemistry 19, 565 (2000).

${ }^{86}$ P.D. Kirchner, W.J. Schaff, G.N. Maracas, L.F. Eastman, T.I. Chappell, and C.M. Ransom, Journal of Applied Physics 52, 6462 (1981).

${ }^{87}$ K. Ikeda and H. Takaoka, Jpn. J. Appl. Phys. 21, 462 (1982).

${ }^{88}$ C.D. Wang and H.C. Lin, Journal of Applied Physics 52, 546 (1981).

${ }^{89}$ W. Mayeda, Digital Signal Processing (Prentice Hall, Englewood Cliffs, NJ, 1993).

${ }^{90}$ H.H. Telle, Laser Chemistry: Spectroscopy, Dynamics and Applications (John Wiley \& Sons, Chichester, West Sussex, England ; Hoboken, NJ, 2007). 


\section{Appendix A: FFT algorithm for decay constant calculation}

An efficient method for determining the exponential decay constant in sampled data was implemented in CRDS by Mazurenka et.al. ${ }^{20}$ The method was adopted from Kirchner et.al. ${ }^{86}$ (for more information see also Ikeda et.al. ${ }^{87}$, and Wang et.al. ${ }^{88}$ ) The method is based on the result that for a continuous exponentially decaying function, $\beta / \omega$ is given by:

$$
\frac{\beta}{\omega}=-\frac{\operatorname{Re}(X(\omega))}{\operatorname{Im}(X(\omega))}
$$

where $X(\omega)$ is the discrete Fourier transform of the time series data, which is assumed to be a decaying exponential (i.e., $x(t)=A e^{-\beta t}+B$ ) with decay constant $\beta$. Thus given any $\omega_{i}$ and $X\left(\omega_{i}\right), \beta$ can be obtained. This Appendix presents the proof of equation (20), and looks at the effect of noise on the estimation of $\beta$. This is similar to that given in Everest and Atkinson ${ }^{70}$ The Discrete Fourier Transformation (DFT) of a discrete function $x(n)$ with period $N$ is given by ${ }^{89}$ :

$$
\begin{aligned}
X(k) & =\sum_{n=0}^{N-1} x(n) e^{-j\left(\frac{2 \pi}{N}\right) n k} \\
\mathrm{k} & =0,1, \ldots \mathrm{N}-1
\end{aligned}
$$

and $x(n)$ can be obtained using the Inverse DFT of $X(k)$ :

$$
x(n)=\frac{1}{N} \sum_{k=0}^{N-1} X(k) e^{j\left(\frac{2 \pi}{N}\right) n k}
$$


If $x(n)$ is the sampled data time series of the general exponentially decaying function, $x(t)=x(t)=A e^{-\beta t}+B$, using a sampling time $t_{s}$, (sampling frequency $\mathrm{f}_{\mathrm{s}}=1 / \mathrm{t}_{\mathrm{s}}$ ) then $x(n)=A \exp \left(-\beta t_{s} n\right)+B$. Then, using eq. (21), the DFT becomes:

$$
\begin{aligned}
& X(k)=\sum_{n=0}^{N-1}\left(A e^{-\beta t_{s} n}+B\right) e^{-j\left(\frac{2 \pi}{N}\right) n k} \\
& =A \sum_{n=0}^{N-1} e^{-\left[j\left(\frac{2 \pi}{N}\right) k+\beta t_{s}\right] n}+B \sum_{n=0}^{N-1} e^{-j\left(\frac{2 \pi}{N}\right) n k}
\end{aligned}
$$

Evaluating the sums:

$$
\begin{aligned}
\sum_{n=0}^{N-1} e^{-a n} & =\frac{1-e^{-a N}}{1-e^{-a}} \\
\sum_{n=0}^{N-1} e^{-\left[j\left(\frac{2 \pi}{N}\right) k+\beta t_{s}\right] n} & =\frac{1-e^{-\left[j\left(\frac{2 \pi}{N}\right) k+\beta t_{s}\right] N}}{1-e^{\left.-j\left(\frac{2 \pi}{N}\right) k+\beta t_{s}\right]}} \\
& =\frac{1-e^{-\left[j\left(\frac{2 \pi}{N}\right) k N+\beta t_{s} N\right]}}{1-e^{-\left[j\left(\frac{2 \pi}{N}\right) k+\beta t_{s}\right]}} \\
& =\frac{1-e^{-[j 2 \pi k+\beta T]}}{1-e^{-\left[j\left(\frac{2 \pi}{N}\right) k+\beta t_{s}\right]}} \\
& =\frac{1-e^{-j 2 \pi k} e^{-\beta T}}{1-e^{-\left[j\left(\frac{2 \pi}{N}\right) k+\beta t_{s}\right]}}
\end{aligned}
$$

So that we have

$$
\sum_{n=0}^{N-1} e^{-\left[j\left(\frac{2 \pi}{N}\right) k+\beta t_{s}\right] n}=\frac{1-e^{-\beta T}}{1-e^{-\left[j\left(\frac{2 \pi}{N}\right) k+\beta t_{s}\right]}}
$$

For the first sum, and for the second sum, 


$$
\begin{aligned}
& \sum_{n=0}^{N-1} e^{-j\left(\frac{2 \pi}{N}\right) n k}=\frac{1-e^{e^{-j\left(\frac{2 \pi}{N}\right) N k}}}{1-e^{-j\left(\frac{2 \pi}{N}\right) k}}=\frac{1-e^{-j 2 \pi k}}{1-e^{-j\left(\frac{2 \pi}{N}\right) k}} \\
& =\frac{1-1}{1-e^{-j\left(\frac{2 \pi}{N}\right) k}} \text { for } k>0 \\
& \sum_{n=0}^{N-1} e^{-j\left(\frac{2 \pi}{N}\right) n k}=\sum_{n=0}^{N-1} e^{0}=N \\
& \text { or } \\
& \sum_{n=0}^{N-1} e^{-j\left(\frac{2 \pi}{N}\right) n k}=N \delta_{k}= \begin{cases}N & k=0 \\
0 & k=1,2 . . N-1\end{cases}
\end{aligned}
$$

So, the DFT becomes:

$$
X(k)=A\left(1-e^{-\beta T}\right)\left(\frac{1}{1-e^{-\beta t_{s}} e^{-j\left(\frac{2 \pi}{N}\right) k}}\right)+B N \delta_{k}
$$

Or

$$
X(k)=A\left(1-e^{-\beta T}\right)\left(\frac{1}{1-e^{-\beta t_{s}} \cos \left(2 \pi \frac{k}{N}\right)+j e^{-\beta t_{s}} \sin \left(2 \pi \frac{k}{N}\right)}\right)+B N \delta_{k}
$$

Multiplying the numerator and denominator by conjugate of denominator:

$$
X(k)=A\left(1-e^{-\beta T}\right)\left(\frac{1-e^{-\beta t_{s}} \cos \left(2 \pi \frac{k}{N}\right)-j e^{-\beta t_{s}} \sin \left(2 \pi \frac{k}{N}\right)}{\left(1-e^{-\beta t_{s}} \cos \left(2 \pi \frac{k}{N}\right)\right)^{2}+e^{-2 \beta t_{s}} \sin ^{2}\left(2 \pi \frac{k}{N}\right)}\right)+B N \delta_{k}
$$

And simplifying: 


$$
X(k)=A\left(1-e^{-\beta T}\right)\left(\frac{1-e^{-\beta t_{s}} \cos \left(2 \pi \frac{k}{N}\right)-j e^{-\beta t_{s}} \sin \left(2 \pi \frac{k}{N}\right)}{1-2 e^{-\beta t_{s}} \cos \left(2 \pi \frac{k}{N}\right)+e^{-2 \beta t_{s}}}\right)+B N \delta_{k}
$$

Taking $\operatorname{Re}\{\mathrm{X}(\mathrm{k}\} / \operatorname{Im}\{\mathrm{X}(\mathrm{k})\}$ for $\mathrm{k}>0$ :

$$
\frac{\operatorname{Re}\{X(k)\}}{\operatorname{Im}\{X(k)\}}=\left(\frac{1-e^{-\beta t_{s}} \cos \left(2 \pi \frac{k}{N}\right)}{-e^{-\beta t_{s}} \sin \left(2 \pi \frac{k}{N}\right)}\right)
$$

For $\mathrm{k} / \mathrm{N}<<1(\mathrm{k}<<\mathrm{N})$,

$$
\begin{aligned}
\frac{\operatorname{Re}\{X(k)\}}{\operatorname{Im}\{X(k)\}} & =\left(\frac{e^{-\beta t_{s}}\left(1-\frac{1}{2}\left(2 \pi \frac{k}{N}\right)^{2}+\cdots\right)-1}{e^{-\beta t_{s}}\left(\left(2 \pi \frac{k}{N}\right)-\frac{1}{3}\left(2 \pi \frac{k}{N}\right)^{3}+\cdots\right)}\right) \approx \frac{e^{-\beta t_{s}}-1}{e^{-\beta t_{s}}\left(2 \pi \frac{k}{N}\right)} \\
& \approx\left(1-e^{\beta t_{s}}\right)\left(\frac{N}{2 \pi k}\right)
\end{aligned}
$$

Defining the decay time constant $\tau=1 / \beta$, as long as $\tau>>t_{s}$ (i.e., decay lasts much longer than a single sample time, then:

$$
\begin{aligned}
\frac{\operatorname{Re}\{X(k)\}}{\operatorname{Im}\{X(k)\}} & =\left(1-e^{\beta t_{s}}\right)\left(\frac{N}{2 \pi k}\right) \\
& \approx\left(1-1-\beta t_{s}-\frac{1}{2}\left(\beta t_{s}\right)^{2}-\cdots\right)\left(\frac{N}{2 \pi k}\right) \\
\frac{\operatorname{Re}\{X(k)\}}{\operatorname{Im}\{X(k)\}} & =-\left(\frac{N}{2 \pi k}\right) \beta t_{s}
\end{aligned}
$$

So that, once $X(k)$ is calculated, we can choose $k=1$, which gives ( 


$$
\beta=-\left(\frac{2 \pi}{T}\right) \frac{\operatorname{Re}\{X(1)\}}{\operatorname{Im}\{X(1)\}}
$$

And, since $\omega_{1}=2 \pi / T$, this is the same as equation (20).

The error in the assumption that $\tau>>t_{s}$ can be easily calculated using equation (30). In fact, we can calculate $\beta$ exactly by solving equation (30) for $\beta$.

$$
\begin{aligned}
& \frac{\operatorname{Re}\{X(1)\}}{\operatorname{Im}\{X(1)\}}=\left(1-e^{\beta t_{s}}\right)\left(\frac{N}{2 \pi}\right) \\
& \beta=\frac{N}{T} \ln \left(1-\left(\frac{2 \pi}{N}\right) \frac{\operatorname{Re}\{X(1)\}}{\operatorname{Im}\{X(1)\}}\right)
\end{aligned}
$$

$$
\begin{aligned}
\beta & =\frac{N}{T} \ln \left(1-\left(\frac{2 \pi}{N}\right) \frac{\operatorname{Re}\{X(1)\}}{\operatorname{Im}\{X(1)\}}\right) \\
& =\left(-\left(\frac{2 \pi}{T}\right) \frac{\operatorname{Re}\{X(1)\}}{\operatorname{Im}\{X(1)\}}+\frac{1}{2} \frac{T}{N}\left(\frac{4 \pi^{2}}{T^{2}}\right)\left(\frac{\operatorname{Re}\{X(1)\}}{\operatorname{Im}\{X(1)\}}\right)^{2}-\frac{1}{3} \frac{T^{2}}{N^{2}}\left(\frac{8 \pi^{3}}{T^{3}}\right)\left(\frac{\operatorname{Re}\{X(1)\}}{\operatorname{Im}\{X(1)\}}\right)^{3}+\cdots\right) \\
& =\hat{\beta}+\frac{1}{2} \frac{T}{N} \hat{\beta}^{2}-\frac{1}{3} \frac{T^{2}}{N^{2}} \hat{\beta}^{3}+\frac{1}{4} \frac{T^{3}}{N^{3}} \hat{\beta}^{4}-\cdots \\
& =\hat{\beta}\left(1+\frac{1}{2}\left(\frac{t_{s}}{\hat{\tau}}\right)-\frac{1}{3}\left(\frac{t_{s}}{\hat{\tau}}\right)^{2}+\frac{1}{4}\left(\frac{t_{s}}{\hat{\tau}}\right)^{3}-\cdots\right)
\end{aligned}
$$

where $\hat{\beta}$ is the estimate of the decay constant, and $\hat{\tau}=1 / \hat{\beta}$ is the estimated time constant. For example, if $\hat{\boldsymbol{\tau}}$ is $12 \mu \mathrm{s}$, and $t_{s}=0.01 \mu \mathrm{s}, t_{s} / \hat{\boldsymbol{\tau}}=0.00083$, and the error is $\approx 0.04 \%$. 


\section{Appendix B: Optical heterodyne detection}

The method of optical heterodyne detection (OHD) is similar to the demodulation of radio frequencies using a mixer (a nonlinear device such as a diode that produces an output containing a term with the product of two oscillating input signals $)^{72}$. OHD, on the other hand, is based on the fact that the response of a photodiode is proportional to the intensity (which is proportional to the electric field squared) of the light hitting the detector, and not the electric field ${ }^{90}$. So if light from two different sources is incident on the photodetector simultaneously, the photodetector response (the output voltage) is:

$$
\begin{aligned}
V(t) & =\gamma\left|E_{1}(t)+E_{2}(t)\right|^{2} \\
& =\gamma\left|\varepsilon_{1} \sin \left(\omega_{1} t\right)+\varepsilon_{2} \sin \left(\omega_{2} t+\Delta \phi\right)\right|^{2}
\end{aligned}
$$

Where $\gamma$ is a proportionality constant, and $E_{1}(t)=\varepsilon_{1} \sin \left(\omega_{1} t\right)$ and $E_{2}(t)=\varepsilon_{2} \sin \left(\omega_{2} t+\Delta \phi\right)$ are the electric field from Source 1 and Source 2, respectively, which oscillate at their respective optical frequencies. The sources have a phase difference of $\Delta \phi$, and constant frequencies of $\omega_{1}$ and $\omega_{2}$, and amplitudes of $\varepsilon_{1}$ and $\varepsilon_{2}$, respectively. Completing the square we have:

$$
\begin{aligned}
V(t) & =\gamma\left[\varepsilon_{1}^{2} \sin ^{2}\left(\omega_{1} t\right)+\varepsilon_{2}^{2} \sin ^{2}\left(\omega_{2} t+\Delta \phi\right)+\varepsilon_{1} \varepsilon_{2} \sin \left(\omega_{1} t\right) \sin \left(\omega_{2} t+\Delta \phi\right)\right] \\
& =\gamma\left[\varepsilon_{1}^{2} \sin ^{2}\left(\omega_{1} t\right)+\varepsilon_{2}^{2} \sin ^{2}\left(\omega_{2} t+\Delta \phi\right)\right]+\gamma \varepsilon_{1} \varepsilon_{2} \sin \left(\omega_{1} t\right) \sin \left(\omega_{2} t+\Delta \phi\right)
\end{aligned}
$$


We can see that the first two terms are just the intensities of the two sources, and we can use the properties of trigonometric identities to replace the last term with the difference of two cosine functions ${ }^{72}$ to simplify:

$$
\begin{aligned}
V(t) & =\gamma\left[\varepsilon_{1}^{2} \sin ^{2}\left(\omega_{1} t\right)+\varepsilon_{2}^{2} \sin ^{2}\left(\omega_{2} t+\Delta \phi\right)+\varepsilon_{1} \varepsilon_{2} \sin \left(\omega_{1} t\right) \sin \left(\omega_{2} t+\Delta \phi\right)\right] \\
& =\gamma\left(\left|E_{1}\right|^{2}+\left|E_{2}\right|^{2}\right)-\frac{\gamma}{2} \varepsilon_{1} \varepsilon_{2} \cos \left(\omega_{1} t+\omega_{2} t+\Delta \phi\right)+ \\
& \frac{\gamma}{2} \varepsilon_{1} \varepsilon_{2} \cos \left(\omega_{1} t-\omega_{2} t-\Delta \phi\right) \\
& =\gamma\left(\left|E_{1}\right|^{2}+\left|E_{2}\right|^{2}\right)-\frac{\gamma}{2} \varepsilon_{1} \varepsilon_{2} \cos \left(\left(\omega_{1}+\omega_{2}\right) t+\Delta \phi\right)+ \\
& \frac{\gamma}{2} \varepsilon_{1} \varepsilon_{2} \cos \left(\left(\omega_{1}-\omega_{2}\right) t-\Delta \phi\right)
\end{aligned}
$$

This leaves us with two oscillating intensities, one at the sum of the oscillation frequencies of the two sources, and the other at the difference of the oscillation frequencies.

Photodetectors typically have bandwidths of up to several $\mathrm{GHz}$, and, since for infra-red wavelengths and below, the oscillation frequency is above $10^{15} \mathrm{~Hz}$, so the first two terms result in a constant (DC component) that is their time average, $\left(\varepsilon_{1} / 2+\right.$ $\left.\varepsilon_{2} / 2\right)$. The frequency $\left(\omega_{1}+\omega_{2}\right)$ term in Equation (37) is also well above the photodetector bandwidth, and is filtered out, leaving the only time-varying detector response at the frequency $\left(\omega_{1}-\omega_{2}\right)$. If this frequency difference is less than the bandwidth of the detector, then the output voltage will oscillate at that frequency, which can be measured with a spectrum analyzer or an oscilloscope.

The application of OHD typically uses a reference laser, which is typically highly stable, low noise, and has a narrow linewidth, to precisely measure the wavelength or 
frequency of a laser-under-test. By tuning the reference laser to be close to the laser under test, OHD measures how far away the laser-under-test is from the reference, and by looking at the frequency spectrum of the photodetector output, the linewidth of the laserunder-test can be determined, assuming it is significantly wider than the reference laser. Another advantage of OHD is that, as seen in equation (37), the amplitude of this oscillation is the product of the amplitude's of the reference and test lasers, so that small test laser intensities are amplified by the reference laser, and can result in a higher signalto-noise ratio of the output voltage.

In our system OHD was used for three applications. The first was to determine the main laser's wavelength (frequency) as the main laser's temperature can current were varied (as described in Section 3.3). The tunable diode laser (New Focus, Model 6324) was used as the reference laser, and it was adjusted until an oscillating signal was observed on the oscilloscope, indicating that the two lasers were close in frequency.

The second application of OHD was for measuring the frequency shift induced by the pulse laser for the optical frequency unlocking, and to verify that the main laser returned to its orginal frequency after the pulse. Both of these measurements are described in Section Chapter 4. OHD was also used to initially determine the wavelength (frequency) of the pulse laser so that the difference in wavelength between the two could be measured.

The third application was to measure the effect of the optical feedback on the linewidth of the main laser with and without optical feedback, and to show that the linewidth narrowed with our optical feedback configuration. 


\section{Appendix C: FPGA Code}

The following is the code used to program the FPGA used for data processing, as

described in Section Chapter 5. In order to program the FPGA, the Xilinx ISE® Design

Suite, Xilinx LogiCORETM IP Fast Fourier Transform core $^{80}$ and the LogiCORETM IP

Divider Generator core ${ }^{81}$ are required.

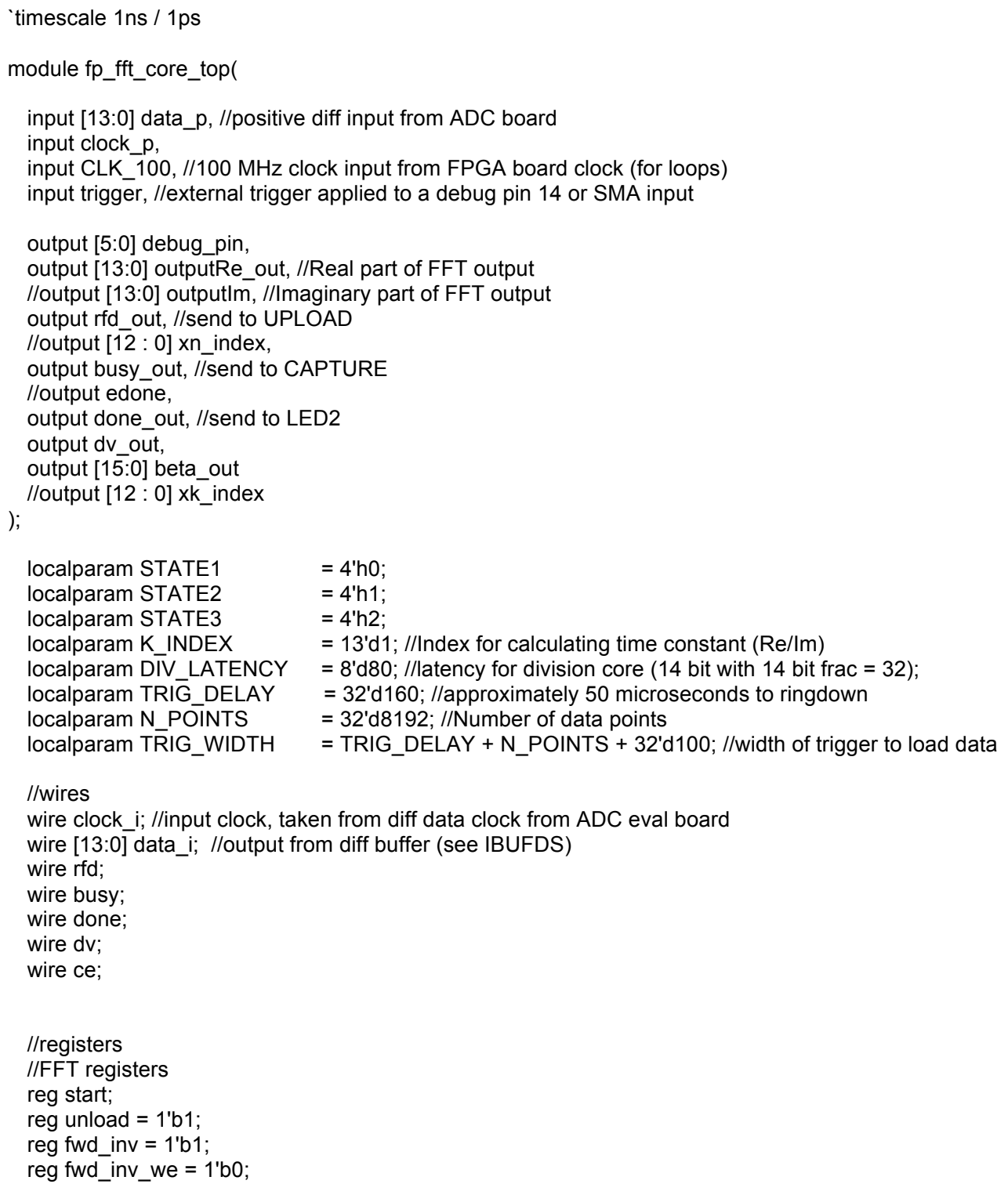


reg $[13: 0]$ scale_sch = 14'b0110_1010_1010_11; //for 8192, 14 values for stage scaling (setting all to 1) reg scale_sch_we $=1$ 'b0;

reg [3:0] state;

reg core_reset $=1$ 'b0;

reg RESET = 1'b1;

reg [5:0] debug_pin_reg;

reg rfd_reg;

reg busy_reg;

reg done_reg;

reg dv_reg;

reg busy_dv_reg = 1 'b0;

reg dv div reg = 1'b0;

reg signed [27:0] outputRe_reg;

reg signed [27:0] outputlm_reg;

reg [15:0] beta_reg;

reg $[7: 0]$ div counter $=8$ 'b0;

reg [31:0] trig_counter $=32$ ' $\mathrm{d} 0$;

reg trigger_delayed $=1$ 'b0;

reg istrigcounting $=1$ ' $\mathrm{b} 0$;

reg trig_reg $=1$ 'b0;

reg [12:0] xk_index_reg;

reg index test reg;

reg [15:0] data_reg;

//Re/lm registers

reg signed [27:0] FFTRe; //

reg signed [27:0] FFTIm; //

reg div_enable_reg = 1 'b0; //

reg div wait reg $=1$ 'b0;

reg [31:0] beta_simplek_reg; //

reg [27:0] ratio_result_reg;

reg [13:0] ratio_frac_result_reg;

//fft wires

wire signed [27:0] outputRe;

wire signed [27:0] outputlm;

wire $[12: 0] \mathrm{xn}$ index;

wire [12:0] xk_index;

wire edone;

//Re/Im division wires for output

wire [27:0] ratio_result;

wire [27:0] ratio_result_abs $=\sim($ ratio_result-28'b1 $)$;

wire [13:0] ratio_frac_result;

wire [13:0] ratio_frac_result_abs $=\sim($ ratio_frac_result-14'b1 $)$;

wire rfd_ratio;

wire div_wait;

wire div_enable;

wire index_test;

//assign output wires

assign debug_pin $=$ debug_pin reg

assign done_out $=$ done_reg;

assign $\mathrm{rfd}$ out $=\mathrm{rfd}$ reg;

assign busy_out = busy_reg;

assign $\mathrm{dv}$ out $=\mathrm{dv}$ reg;

assign clock_i $=$ clock_p;

assign data $i=$ data $p$;

assign outputRe_out = outputRe_reg[27:14];

assign beta_out[15:13] = ratio_result_reg[2:0]; //take 3 LSB bits of integer

assign beta_out[12:0] = ratio_frac_result_reg[12:0]; //'14th bit is 0 for abs

assign div_wait = div_wait_reg;

assign div_enable = div_enable_reg;

//enable fft when triggered, or while data is valid

assign ce = trigger_delayed|busy_dv_reg|dv_div_reg|div_wait;

assign index_test $=$ index_test_reg;

//end assignments 


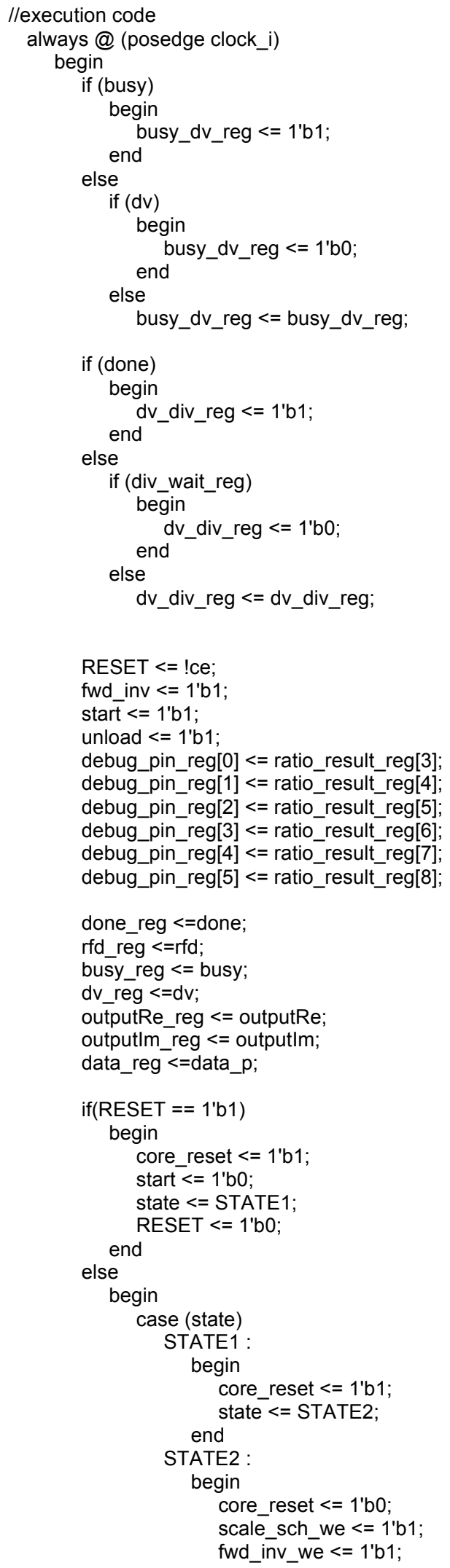




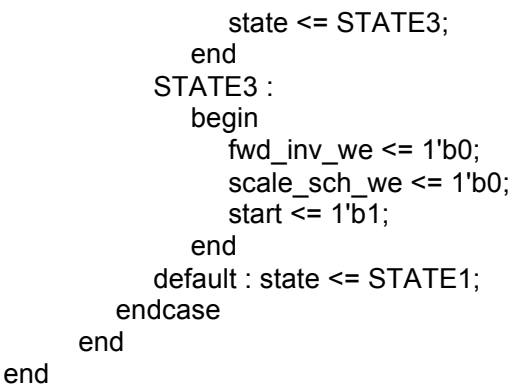

//Triggering Delay counter always@ (posedge clock_i) begin

if (trigger\&!(ce|istrigcounting))

//if external trigger input is high, then start counting for delay

if (trig_reg|istrigcounting)

trig_counter $=$ trig_counter+1;

//set flag to indicate externally triggered

if (trig_counter $>0$ )

istrigcounting $=1 \mathrm{\prime} b 1$;

else

istrigcounting $=1$ ' $b 0$;

//if counter reaches delay time, then set software trigger to set fft $\mathrm{CE}$ if (trig_counter > TRIG_DELAY) 


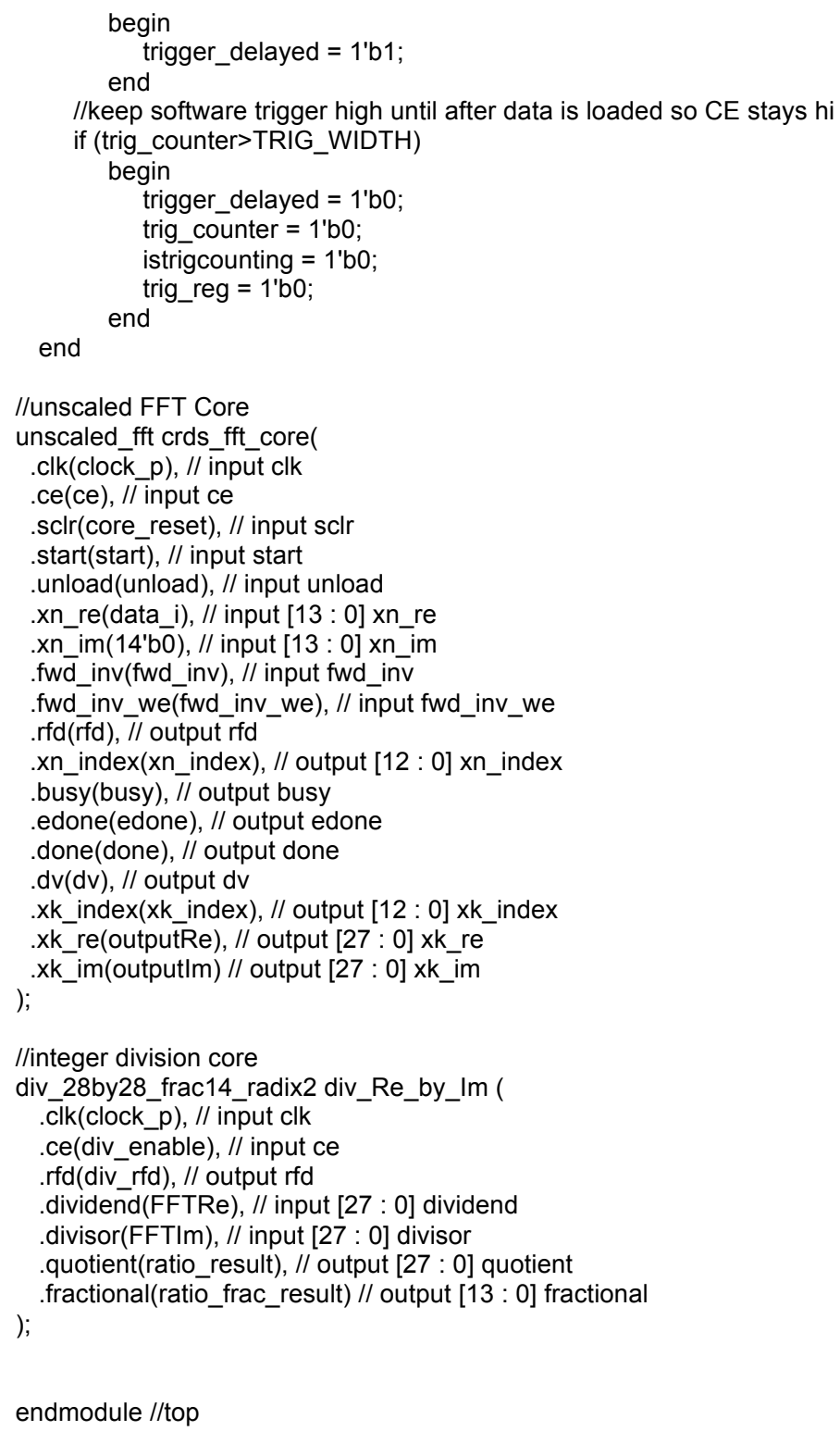

endmodule //top 


\section{Appendix D: Arduino Due Code for FPGA}

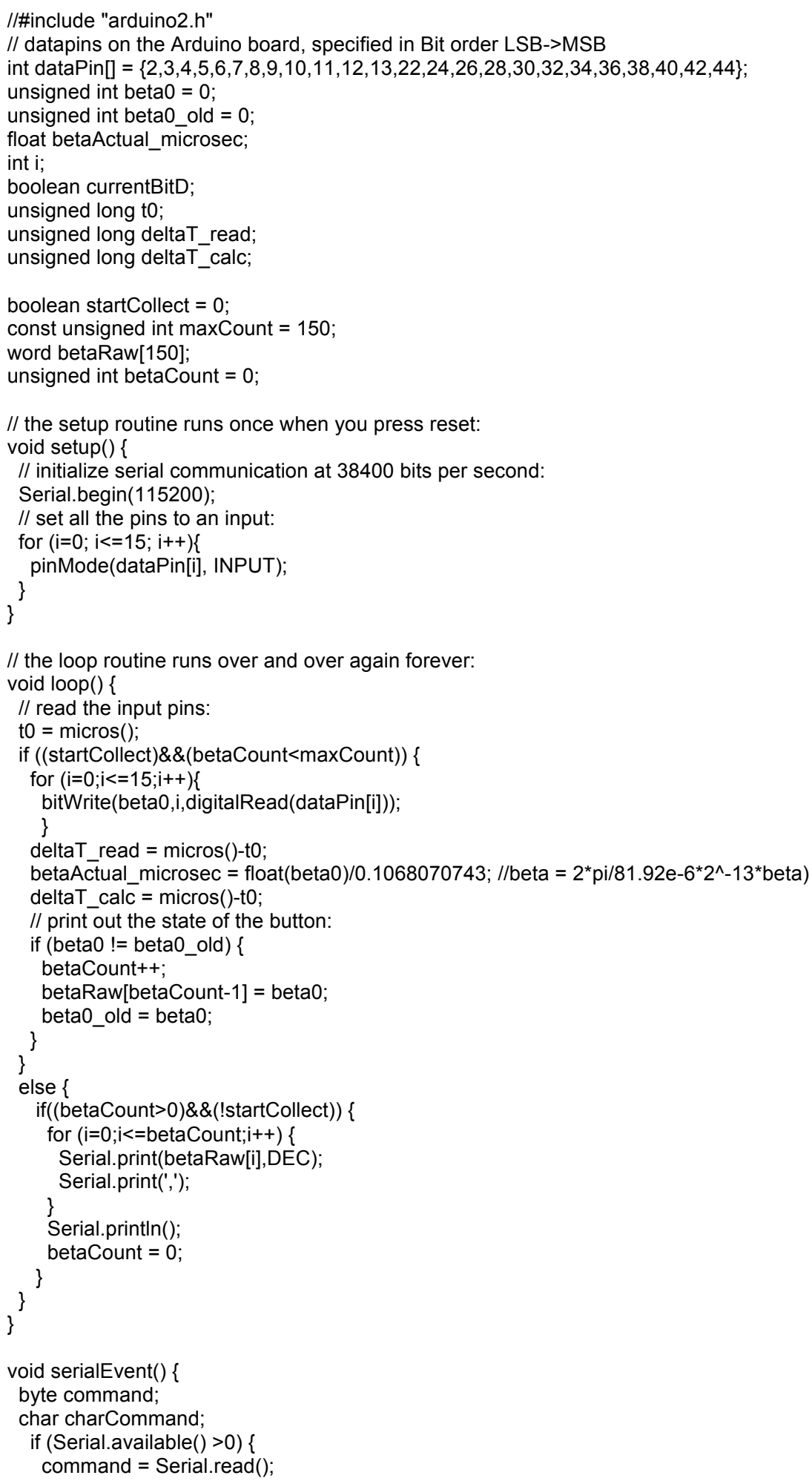




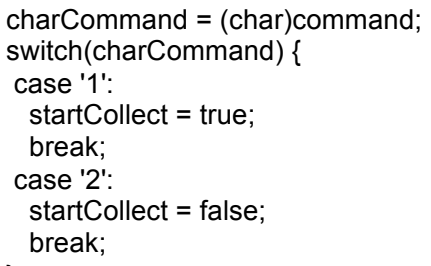




\section{Appendix E: Arduino Code for DAC1220}

What follows is the code used to program the Aduino Uno (R3) to control the laser current via the DAC1220 digital-to-analog converter, as described in Section 3.3.

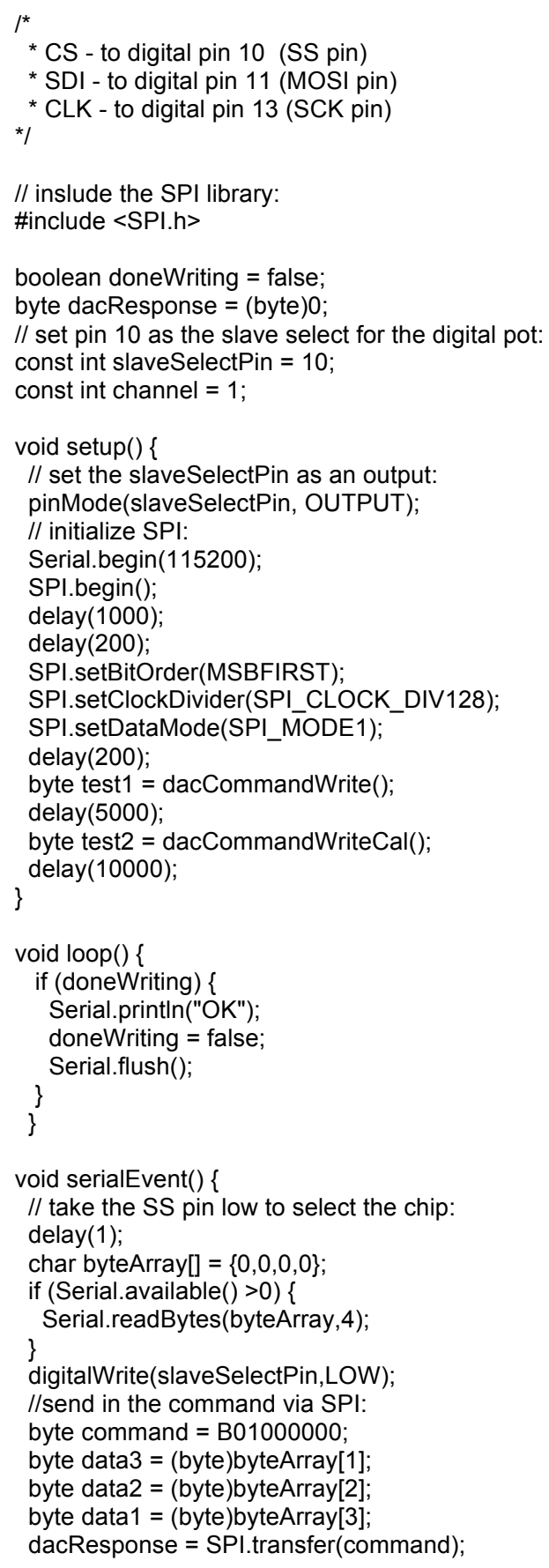




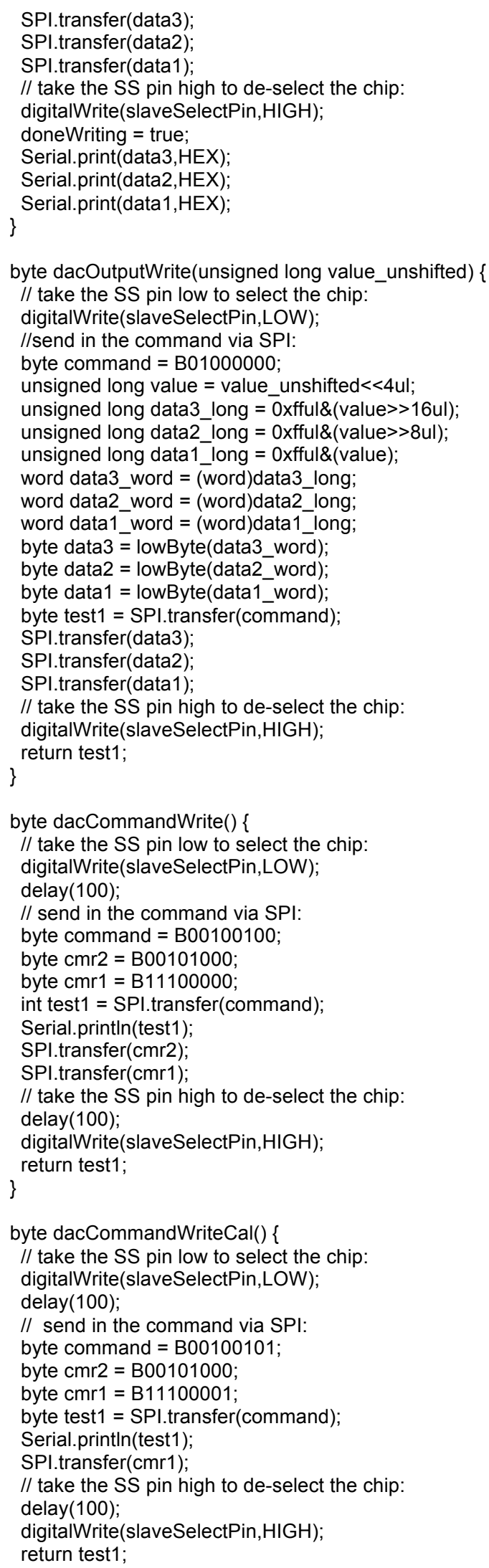


byte dacCMR2Read() \{

// take the SS pin low to select the chip: digitalWrite(slaveSelectPin,LOW);

// send in the command via SPI:

byte command = B10000101;

byte test 1 = SPI.transfer (command);

Serial.println(test1);

delay(100);

digitalWrite(slaveSelectPin,HIGH);

return test 1 ;

\} 


\section{Appendix F: LabView Front End}

The image shown is the user interface for the LabView code which controls the CRDS system, as described in Section 6.1.

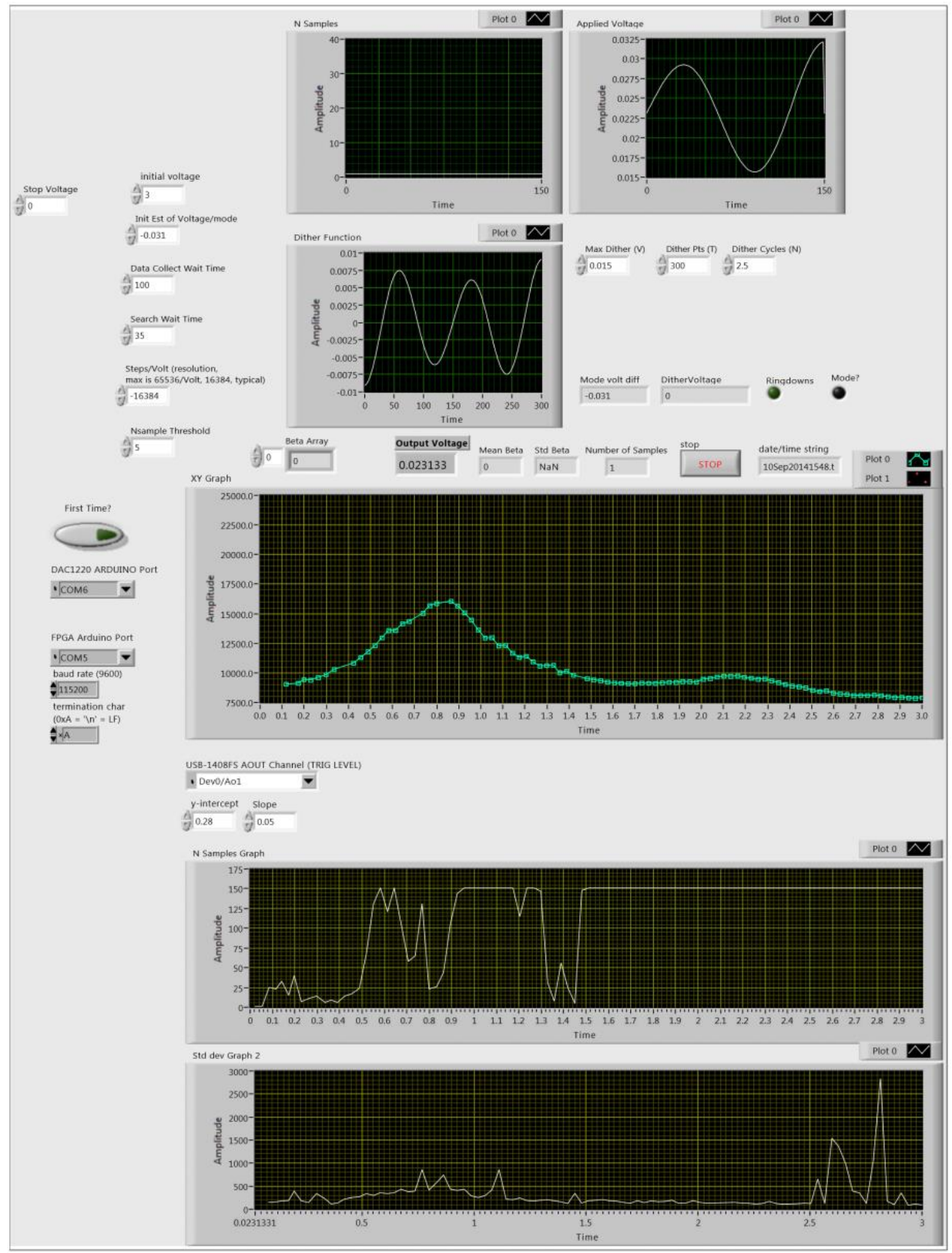

Figure F-1. User interface for the LabView code which controls the CRDS system and collects the data. 